\title{
Development and Validation
}

of a Cleanup Method for

Hydrocarbon Containing

Samples for the Analysis of

Semivolatile Organic Compounds
E. W. Hoppe
M. J. Steele
R. W. Stromatt
T. E. Jones
I. A. Campbell

April 1992

Prepared for the U.S. Department Energy under Contract DE-AC06-76RLO 1830

Pacific Northwest Laboratory Operated for the U.S. Department of Energy by Battelle Memorial institute 


\title{
DISCLAIMER
}

This report was prepared as an account of work sponsored by an agency of the United States Covernment. Neither the United States Government nor any agency thereof, nor Battelle Memorial Institute, nor any of their employees, makes any warranty, expressed or implied, or assumes any legal liability or responsibility for the accuracy, completeness, or usefulness of any information, apparatus, product, or process disclosed, or represents that its use would not infringe privately owned rights. Reference herein to any specific commercial product, process, or service by trade name, trademark, manufacturer, or otherwise does not necessarily constitute or imply its endorsement, recommendation, or favoring by the United States Government or any agency thereof, or Battelle Memorial Institute. The views and opinions of authors expressed herein do not necessarily state or reflect those of the United States Government or any agency thereof.

\author{
PACIFIC NORTHWEST LABORATORY \\ operated by \\ BATTELLE MEMORIAL INSTITUTE \\ for the \\ UNITED STATES DEPARTMENT OF ENERGY' \\ under Contract DE-ACO6-76RLO 1830
}

Printed in the United States of America

Available to DOE and DOE contractors from the

Office of Scientific and Technical Information, P.O. Box 62, Oak Ridge, TN 37831; prices available from (615) 576-8401. FTS 626-8401.

Available to the public from the National Technical Information Service, U.S. Department of Commerce, 5285 Port Royal Rd., Springfield, VA 22161. 
PNL- -8098

DE92 013759

DEVELOPMENT AND VALIDATION OF A C.LEANUP METHOD FOR HYDROCARBON CONTAINING SAMPLES FOR THE ANALYSIS OF SEMIVOLATILE ORGANIC COMPOUNDS

E. W. Hoppe

R. W. Stromatt

J. A. Campbell

M. J. Steele

T. E. Jones

Apri1 1992

Prepared for

the U.S. Department of Energy

under Contract DE-AC06-76RLO 1830

Pacific Northwest Laboratory

Richland, Washington 99352 


\section{EXECUTIVE SUMMARY}

Samples obtained from the Hanford single shell tanks (SSTs) are contaminated with normal paraffin hydrocarbon (NPH) as hydrostatic fluid from the sampling process or can be native to the tank waste. The contamination is usually high enough that a dilution of up to several orders of magnitude may be required before the sample can be analyzed by the conventional gas chromatography/mass spectrometry methodology. This can prevent detection and measurement of organic constituents that are present at lower concentration levels. To eliminate or minimize the problem, a sample cleanup method has been developed and validated and is presented in this document.

The sample is first extracted following Pacific Northwest Laboratory procedure PNL-ALO-120 or PNL-AL0-344. An aliquot of the extract is added to water, which is then made basic, and the base-neutral compounds are extracted into methylene chloride. The aqueous phase is made acidic, and the acidic compounds are extracted into methylene chloride. The base-neutral fraction is solvent exchanged into cyclohexane, and is eluted through a commercial prepacked silica gel column. The NPH is eluted from the column with pentane, which is followed by elution of the base-neutrals with successively more polar solvents. This fraction and the acid fraction are concentrated, and are ready for analysis.

The effectiveness of this cleanup method was demonstrated for all of the U.S. Environmental Protection Agency's (EPA) semivolatile target list compounds. Both blanks and single shell tank samples spiked with all target compounds and NPH were used in this development and validation study. The NPH removal averaged $94 \%$ for samples from SST B110 and $97 \%$ for samples from tank U110. The recoveries were good for most of the target compounds and satisfactory for al1, although they were lower for the more troublesome compounds. Advisory quality control limits were established for the surrogate and matrix spike compounds. As more data are obtained using this cleanup method, required $Q C$ limits will be put in place, and a better definition of cleanup effectiveness can be established. 


\section{CONTENTS}

EXECUTIVE SUMMARY . . . . . . . . . . . . . . . . . . . . . i i . . . . . . INTRODUCTION . . . . . . . . . . . . . . . . . . . . . . . . 1

INITIAL PNL PROCEDURE GC/MS ANALYSES . . . . . . . . . . 3

EPA SW-846 METHOD 3630 PROCEDURE GC/MS ANALYSES . . . . . . . . 4

FINAL PNL PROCEDURE GC/MS ANALYSES . . . . . . . . . . . . . 4

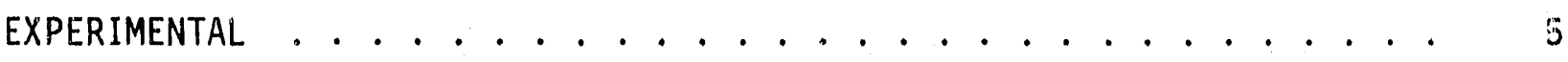

PROCEDURES ...................... 5

Preliminary Investigation ............. 5

Initial Cevelopment and Validation Study . . . . . . . . 6

Final Development and Validation Study .......... 8

ANALYSIS ...................... 8

RESULTS. . . . . . . . . . . . . . . . . . . 11

PRELIMINARY INVESTIGATION . . . . . . . . . . . . . . . . 11

INITIAL DEVELOPMENT AND VALIDATION STUDY . . . . . . . . . 11

FINAL DEVELOPMENT AND VALIDATION STUDY . . . . . . . . . . 13

DISCUSSION . . . . . . . . . . . . . . . . . . . . 15

PRELIMINARY INVESTIGATION . . . . . . . . . . . . . . 15

INITIAL DEVELOPMENT AND VALIDATION STUDY . . . . . . . . . 15

FINAL DEVELOPMENT AND VALIDATION STUDY . . . . . . . . . . . 19

METHOD ADVISORY CONTROL LIMITS . . . . . . . . . . . 20

CONCLUSIONS . . . . . . . . . . . . . . . . . . . . . . 23

REFERENCES ........................... 25 
APPENDIX A - PNL-ALO-120, PROCEDURE FOR EXTRACTION OF SINGLE SHELL TANK SAMPLES FOR THE ANALYSIS OF

SEMIVOLATILE ORGAIIIC COMPOUNDS

APPENDIX B - PNL-ALO-122, CLEANUP OF HYDROCARBON CONTAINING SAMPLES FOR THE ANALYSIS OF SEMIVOLATILE ORGANIC COMPOUNDS, Revision 1.0

B. 1 


\section{FIGURES}

1 Flow Diagram of the Initial Development and Validation Study . . . . 26

2 Simplified Schematic of the Column Cleanup Procedures . . . . . . . 27

3 GC/FID Chromatogram of Normal Paraffin Hydrocarbon . . . . . . . 28

4 GC/FID Chromatogram of NPH Through Silica Column Eluted with Hexane .. . . . . . . . . . . . . . . . . 29

5 GC/FID Chromatogram of Methylene Chloride: Hexane Elution of NPH Following Hexane Elution ............ 30

6 GC/FID Chromatogram of the Standard Mix Containing NPH, Base Neutral Mix 2, and AHs Through Silica Column Eluted with Hexane . . 31

7 GC/FID Chromatogram of the Standard Mix Containing NPH, Base Neutral Mix 2, and PAHs Through Silica Column Eluted with Methylene Chloride: Hexane and then Methylene Chloride After Original Elution with Hexane 


\section{TABLES}

1 Calculated Percent Recoveries for Selected Compounds by GC/FID, Preliminary Investigation ... . . . . . . . . . . .

2 Percent Recovery Results for Reagent Blank, Initial PNL Cleanup Method . . . . . . . . . . . . . . . . . 34

3 Percent Recovery Results for Method Blank, Initial PNL Cleanup Method ................ . 36

4 Percent Recovery Results for Blank Spike, Initial PNL Cleanup Method . . . . . . . . . . . . . . . . . .

5 Percent Recovery Results for Blank Spike Duplicate, Initial PNL Cleanup Method . . . . . . . . . . . . . . . . . 40

6 Percent Recovery Results for Method B1ank, EPA 3630 Cleanup Method . . . . . . . . . . . . . . . . . . 42

7 Percent Recovery Results for B1ank Spike, EPA 3630 Cleanup Method . . . . . . . . . . . . . . . . . . .

8 Percent Recovery Results for Blank Spike Duplicate, EPA 3630 Cleanup Method .....................

9 Percent Recovery Results for Blank Spike, Initial PNL Cleanup Method, Followed by Increased Methylene Chloride Elution

10 Percent Recovery Results for Blank Spike with NPH, Initial PNL Cleanup Method, Followed by Increased Methylene Chloride Elution

11 Percent Recovery Results for Blank Spike with NPH Duplicate, Initial PNL Cleanup Method, Followed by Increased Methylene

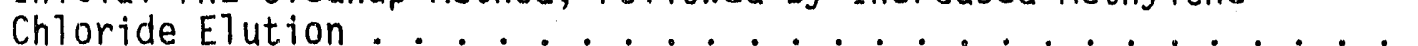

12 Percent Recovery Results for B1ank Spike, EPA 3630 Cleanup

Method, Followed by Methylene Chloride Elution . . . . . . . . .

13 Percent Recovery Results for Blank Spike with NPH, EPA 3630

Cleanup Method, Followed by Methylene Chloride Elution.

14 Percent Recovery Results for Blank Spike Duplicate with NPH, EPA 3630 Cleanup Method, Followed by Methylene Chloride Elution

15 Results for Single She11 Tank B110 Using Initial PNL. Cleanup Method 
16 Percent Recovery Results for Single She11 Tank B110 Matrix Spike, Initial PNL Cleanup Method . . . . . . . . . 62

17 Percent Recovery Results for Single Shell Tank Bl10 Matrix Spike Duplicate, Initial PNL Cleanup Method . . . . . . . . . . . 64

18 Results for Single Shell Tank U110 Using Initial PNL Cleanup Method . . . . . . . . . . . . . . . . 66

19 Percent Recovery Results for Single Shel1 Tank U110 Matrix Spike, Initial PNL Cleanup Method .............. 68

20 Percent Recovery Results for Single She11 Tank U110 Matrix Spike Duplicate, Initial PNL Cleanup Method .......... 70

21 Results for Single She11 Tank B110 Using Initial PNL Cleanup Method . . . . . . . . . . . . . . . . 72

22 Percent Recovery Results for Single She11 Tank B110 Matrix Spike, EPA $3630 \mathrm{Cleanup} \mathrm{Method} \mathrm{..............} 74$

23 Percent Recovery Results for Single Shell Tank B110 Matrix Spike Duplicate, EPA 3630 Cleanup Method ............. 76

24 Comparison of the Effect of NPH in Blank Spikes on Cleanup Method Recoveries Including Methylene Chloride Elution . . . . . . 78

25 Percent Recovery Results for Blank Spikes, Following Soil Extraction Procedure Prior to Final PNL Cleanup Method . . . . . . . 80

26 Percent, Accuracy, and Control Limit Data from Blank Spikes Following Soil Extraction Procedure and Prior to Final PNL Cleanup Method.

27 Percent Recovery Results for B7ank Spikes Numbers 1 and 2 Using the Finat PNL Cleanup Method . . . . . . . . . . . .

28 Percent Recovery Results for B1ank Spikes Numbers 3, 4, and 5 Using the Final PNL Cleanup Method . ............ 86

29 Percent Recovery Results for Blank Spikes Numbers $6,7,8$ and 9 Using the Final PNL Cleanup Method . . . . . . . . . . . . 88

30 Precision Accuracy, and Control Limit Data from BTank Spikes Using the Final PNL Cleanup Method . . . . . . . . . . . . 90

31 Recovery Loss from the Use of Final PNL Cleanup Procedure . . . . . 92 
32 Calculation for Estimating Mean Recoveries for Compounds Spiked in Single Shell Tank Samples B110 and U110 Using the

Final PNL cleanup Procedure (Mean Recovery $=$ P2BN-(NMS-BN -

Blank BN) + Acid) . . . . . . . . . . . . . . . 94

33 Estimated Precision, Accuracy and Control Limits for SST

Semivolatile Organic Analysis . . . . . . . . . . . . 96 


\section{INTRODUCTION}

Analysis of samples for organic compounds as part of the single shell tank (SST) chemical characterization has been compromised by the presence of large amounts of normal paraffin hydrocarbons (NPH). NPH is made up of straight chain fully saturated hydrocarbons primarily in the C12 to C14 range and used as a hydrostatic fluid to keep the bore hole open as core sample segments are removed from the waste tanks. The use of NPH as a hydrostatic fiuid leads to contamination of the core segments. In addition, many tank wastes contain substaritial quantities of NPH which are native to the sample itself. The presence of the high NPH concentration in the samples, whether introduced by sampling or native to the sample, can lead to significant problems in the analyses performed by gas chromatography/mass spectrometry (GC/MS). When performing GC/MS analysis, dilutions often up to several orders of magnitude were necessary to prevent overloading the analytical system. Thus, detection of many less prominent sample constituents was not possible.

Attempts to resolve the difficulties associated with high levels of NPH must be twofold. One approach is to attempt to find another suitable hydrostatic fluid that does not impact sample analyses or to identify a new sampling process that does not require a hydrostatic fluid. This would eliminate NPH as a contaminant and would allow determination of NPH native to the sample. The second approach is to develop a laboratory separations cleanup process that allows removal of native and contaminant NPH from SST samples without compromising the semivolatile organic analyses. This document deals with minimizing the NPH problems associated with the semivolatile analysis utilizing a sample cleanup process.

The approach for separation of $\mathrm{NPH}$ from the analytes of interest in the. semivolatile fraction is based on selective retention of certain components within one phase while the other component is transferred to a second phase. Ideally, one would obtain a complete separation of NPH from the analytes of interest. However, since the separations are based on thermodynamic and kinetic processes that are dependent upon the chemical characteristics of the various compounds and surfaces of the substrates, one must balance the 
complete removal of NPH against the losses of the analytes of interest. Thus, as the chemical characteristics of the analytes of interest approach those of NPH, primarily polarity in this case, the separation becomes more difficult. The approach then must be to remove adequate amounts of NPH and evaluate the recovery of the analytes of interest.

The cleanup method described in this document is employed following extraction of the waste or soll using other procedures such as those provided by the U.S. Environmental Protection Agency (EPA). Extraction procedures used at the Analytical Chemistry Laboratory at Pacific Northwest Laboratory $(P N L)^{(a)}$ are derived directly from EPA Contract Laboratory Program (CLP) Statement of Work (SOW) 2/88 (e.g., PNL method PNL-ALO-344) or from EPA Solid Waste Division methods SW-846 (e.g., method PNL-ALO-120). In the PNL cleanup method, an altquot of the sample extract obtained from a preceding extraction procedure is added to a volume of reagent water. The $\mathrm{pH}$ of the water is adjusted to $>11$, and the base-neutral fraction is extracted into methylene chloride. The $\mathrm{pH}$ of the aqueous phase is then adjusted to $<2$, and the acid fraction is extracted into methylene chloride. Following the extract's concentration and exchange into cyclohexane, the base-neutral fraction is eluted through a silica gel column (an amorphous silica with weakly acidic properties) with pentane. The pentane containing the NPH fraction is discarded, and the analytes are eluted from the column with methylene chloride, methylene chloride/acetone, and acetone. This fraction and the acid fraction from the first cleanup step are concentrated and are then ready for analysis.

The study described in this document consists of a preliminary investigation performed by the Chemical Sciences Department followed by two sets of development and validation experiments performed by the Analytical Chemistry Laboratory at PNL. The preliminary investigation qualitatively assessed the removal of NPH and the recovery of a limited set of semivolatile compounds. The first validation portion of the work presented in this document consisted of testing the proposed procedure based on the preliminary study by performing an extraction, cleanup, and analyses on blank-spikes, blank-spikes with NPH,

(a) Operated for the U.S. Department of Energy by Battelle Memorial Institute linder Contract DE-AC06-76RLO 1830. 
and two samples with spike and NPH. For these studies, the spike contained a11 the EPA CLP SOW $2 / 88$ semivolatile organic target compounds. The final methylene chloride/acetone and acetone column wash steps described in the preceding paragraph were not used in the first portion of the validation studies. However, a final wash step using additional methylene chloride in an attempt to improve analyte recoveries was added for selected samples. In addition, the EPA SW-846 method 3630 was performed along with the PNL method to provide a comparison. The results were favorable, but conditions were not optimum. Consequent7y, in the second portion or the study, the PNL procedure was slightly modified and tested on nine blank-spikes with NPH. Changes tested were the use of commercial pre-packed silica columns and use of a more polar column wash in the final step of the column elution.

The GC/MS analyses were performed on aliquots of sample residues after appropriate steps of the procedures such that analyte recoveries could be identified at each key step of the procedure. A flow diagram of the study and the sampling points for analysis during the procedure is shown in Figure 1. These analyses were performed as follows:

\section{INITIAL PNL PROCEDURE GC/MS ANALYSES}

- after the initial extraction and concentration

- after liquid/liquid base-neutral partition and concentration

- after liquid/liquid acid partition and concentration

- after solvent exchange and silica gel column cleanup steps arid concentration

- after the additional methylene chloride elution step of the cleanup column and concentration when designated.

\section{EPA SW-846 METHOD 3630 PROCEDURE GC/MS ANALYSES}

- after the initial extraction and concentration

- after liquid/liquid base-neutral partition followed by liquid/ liquid neutral back-extraction and concentration

- after liquid/liquid acid partition and concentration 
- after solvent exchange and silica gel column cleanup steps and concentration

- after the additional methylene chloride elution step of the cleanup column and concentration when designated.

\section{FINAL PNL PROCEDURE GC/MS ANALYSES}

In the second set of experiments using the modified PNL procedure, not all of the analyses were performed as for the first set. The analyses after base-neutral partitioning was omitted because assessment of this step had a 7 ready been adequately defined. Analyses performed for this work were as follows:

- after the initial extraction and concentration

- base-neutral fraction after all solvent exchange and cartridge -? eanup steps and concentration

- acid fraction after the Tiquid/7iquid partition and concentration. 


\section{EXPERIMENTAL}

The following discussion describes the experiments that were conducted during the preliminary investigation, and the initial and final studies for the development and validation of a method for removing interfering hydrocarbons from samples prior to analysis for semivolatile oinganic compounds. Included are descriptions of the sample processing procedures and analysis operations.

\section{PROCEDURES}

The sample preparation and cleanup procedures utilized in these studies are described in this section. Included in this description are the procedures used for the preliminary investigation, the PNL and the EPA procedures in the initial development and validation studies, and the modified PNL procedure used in the final stidies.

\section{Preliminary Investigation}

In the following discussion, a description of the sample preparation and cleanup used in the preliminary investigation to establish the feasibility of the method is given.

Sample Preparation: Standards were obtained from Supelco, Inc. (Bellefonte, Pennsylvania) in methylene chloride solvent. They were concentrated by nitrogen blow down and redissolved in hexane. NPH was obtained from Westinghouse Hanford Company. A $5 \mathrm{~mL}$ aliquot was diluted with $20 \mathrm{~mL}$ of hexüne.

Cleanup: 25-30 grams of 100-200 mesh silica gel (Mallinckrodt) was heated at $120^{\circ} \mathrm{C}$ for 12 hours. After cooling, $20 \mathrm{~mL}$ of methylene chioride was added to form a slurry. The slurry was then added to a $2.5 \mathrm{~cm} \mathrm{I.D.} \times 25 \mathrm{~cm}$ glass column and eluted with an additional $50 \mathrm{~mL}$ of methylene chloride. The column was then washed with $100 \mathrm{~mL}$ of hexane. Approximately 10 grams of sodium sulfate, dried at $400^{\circ} \mathrm{C}$ overnight, was placed at the top of the column. After the sample, in hexane, was placed on the column, $75 \mathrm{~mL}$ of hexane was passed through the column and collected for NPH analysis. Then $70 \mathrm{~mL}$ of 
methylene chloride/hexane (2:3) was passed through the column and then finally $30 \mathrm{~mL}$ of methylene chloride was added. The methylene chloride alutions were combined and concentrated for analyte analysis.

Initial Development and Validation Study

The sample preparation and cleanup procedures for both the initial PNL and the EPA method are described in the following discussion. The initial PNL cleanup described in this section is essentially that proposed by the preliminary investigation.

Initial extraction: The initial extraction followed the PNL method PNLALO-120 which is based on EPA methodologies. The PNL-ALO-120 method, as applied to SST samples, assumes a medium level extraction because of sample radioactivity and limited sample availability. The sample $\mathrm{pH}$ is adjusted as necessary to within 5-11. Sodium sulfate and the appropriate spiking compounds are added, and then the methylene chloride. After ultrasonic extraction, the extract is separated from the sample by filtration or centrifugation. The sample extract is aliquoted for the subsequent cleanup. A copy of method PNL-ALO-120 is included in Appendix A.

Initial PNI cleanup: An aliquot of the sample extract obtained from the initial extraction is added to a volume of reagent water. The $\mathrm{pH}$ of the water is adjusted to $>11$, and the base-neutral fraction is extracted three times into methylene chloride. The $\mathrm{pH}$ of the aqueous phase is then adjusted to $<2$, and the acid fraction is extracted three times into methylene chloride. Following the extract's concentration and exchange into hexane, the base-neutral fraction is added to a pentane pre-washed silica gel column packed as described previously in the initial study. Pentane is then added to remove the NPH, and after elution is complete, the hydrocarbon fraction is discarded. The analytes are eluted from the column with successive additions of pentane/ methylene chloride and methylene chloride. For selected samples, an addi.. tional column elution of methylene chloride was employed because of observed remaining color. This fraction and the acid fraction from the first cleanup step are concentrated, and are then ready for analysis. 
A simplified schematic of the cleanup procedure is shown in Figure 2. The PNL cleanup procedure is given in detail in Appendix $B$ of this documer.t. The procedure as it is written represents this initial development and validation study. The procedure will be modified later to represent conditions developed by the second development and validation test set, and to reflect experience gained with additional sample analyses.

EPA Method 3630 cleanup: This method does not contain specific procedures for liquid/liquid partitioning. However, the following describes a partitioning similar to that used in various EPA procedures where the intent is to isolate analytes based on their relative polarities.

An aliquot of the sample extract obtained from the initial extraction is added to a volume of reagent water. The $\mathrm{pH}$ of the water is adjusted to $>11$, and the base-neutral fraction is extracted three times into methylene chloride. The $\mathrm{pH}$ of the aqueous phase is then adjusted to <2, and the acid fraction is extracted three times into methylene chloride and is ready for concentration and subsequent analysis. The methylene chloride containing the baseneutral fraction is then added back to the aqueous phase which is still at pH $<2$. The neutral fraction is then extracted three times and is concentrated, exchanged in cyclohexane, and is ready for the silica gel column cleanup procedure. The aqueous phase, following the extraction of the neutral components and still containing the bases, is made basic to $>11$ and extracted again three times with methylene chloride. This organic phase containing the basic compounds is concentrated and is ready for analysis.

The neutral fraction contained in $2 \mathrm{~mL}$ of cyclohexane is then passed through a $1.0 \times 25 \mathrm{~cm}$ column containing about 10 grams 100-200 mesh preactivated silica gel that was pre-washed with pentane. The column is eluted with $40 \mathrm{~mL}$ pentane, which is then discarded. The neutral fraction is then eluted from the column with methylene chloride/pentane. This solution is concentrated and is then ready for analysis. 


\section{Final Development and Validation Study}

The cleanup procedure described in the preceding section was modified to eliminate problems observed when performing the initial development and validation studies. The modified cleanup procedure is described in the following discussion.

Initial extraction: The initial extraction was performed in the same manner as for the initial validation study.

Final PNL Cleanup: An aliquot of the inftial extraction was separated inco acid and base-neutral fractions employing liquid/liquid partitioning as described in the initial validation study. For the column cleanup portion of the procedure, pre-packed disposable polypropylene columns (Analytichem International-Varian) were employed. These columns are essentially the body of a 60 cc disposable syringe, approximately $2.5 \mathrm{~cm}$ I.D. $X 15 \mathrm{~cm}$ long, packed with 10 grams of 100-200 mesh silica get. The columns were activated at $120^{\circ} \mathrm{C}$ for 12 hours. The column void volume, about $20 \mathrm{~mL}$, was determined by weight using methylene chloride to determine the proper eluting volumes to be used during the cleanup procedure. About $1 \mathrm{~cm}$, approximately 4 grams, of sodium sulfate that had been conditioned at $400^{\circ} \mathrm{C}$ for 8 hours was added to the column. The column was then pre-eluted using $25 \mathrm{~mL}$ of methylene chloride followed by $40 \mathrm{~mL}$ of pentane, which were discarded. The base-neutral extraction residue that had been exchanged into about $2 \mathrm{~mL}$ hexane was added to the column. The colurnn was then eluted with $25 \mathrm{~mL}$ of pentane and this eluent containing the NPH was discarded. The column was then eluted with $25 \mathrm{~mL}$ each of pentane/methylene chloride (1:1), methylene chloride, methylene chioride/acetone (1:1), and finally acetone. All of these fractions were combined and concentrated for analysis.

\section{ANALYSIS}

The instrumentation used in the preliminary investigation and the initial and final PNL sample cleanup studies are described in the following section. Alsc included are descriptions of the instrumentation calibrations, the reagents used, and the analysis procedures. 
Preliminary Investiqation Instrumentation: A Hewlett-Packard 5880 gas chromatograph equipped with a flame ionization detector was utilized to analyze these samples. The GC injector and detector were operated at $300^{\circ} \mathrm{C}$. The oven temperature program began at $50^{\circ} \mathrm{C}$ for 1 minute, then ramped to $300^{\circ} \mathrm{C}$ at a rate of $8^{\circ} \mathrm{C} /$ minute, and held at $300^{\circ} \mathrm{C}$ for 10 minutes.

Initial and Final Development and Validation Instrumentation: The GC/MS used for this work was a Hewlett-Packard system consisting of an HP..5890 GC

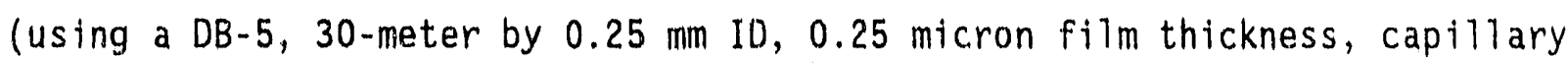
column), an HP-5790 MS, an HP-7673 autosampler, the HP-1000 series A system controller/data acquisition/data processor system, and associated peripherals.

Calibration: The mass spectrometer mass calibration and tune parameters were confirmed with a DFTPP analysis according to EPA CLP SOW 2/88 specifications. A single level continuing calibration was performed for the CLP target list compounds each day of operation.

The calibration for the NPH was perforined at three concentrations: 50 , 100 , and $150 \mu \mathrm{g} / \mathrm{mL}$ total $\mathrm{NPH}$. The extracted ion used for the quantitation was mass 43. Mass 57 was used to resolve ambiguities. The sum of the peak areas for the NPH constituents was used to calculate a single response factor for the NPH, since individual constituent concentrations for the NPH used as spike material for this work were not known.

The area percentages for the NPH constituents were:

$$
\begin{aligned}
& \text { dodecane }-18 \% \\
& \text { tridecane }-48 \% \\
& \text { tetradecane }-33 \% \\
& \text { pcintadecane }-1+\%
\end{aligned}
$$

The internal standard 1,4-Dichlorobenzene- $d_{4}$ was used for NPH quantitation because its non-deuterated analog compound, 1,4-Dichlorobenzene, behaved similarly in the cleanup procedure to the NPH.

Reagents: All the solvents used in this work were of pesticide quality or equivalent. The target compounds for the spikes and instrument calibration were prepared from Supelco Analytical Reference Standards for EPA Contract 
Laboratory Program Methods mixes. The stock spike solution was prepared by combining the Supelco mixes such that each analyte was at $200 \mu \mathrm{g} / \mathrm{mL}$. The $\mathrm{NPH}$ was obtained from Westinghouse Hanford Company. The NPH was prepared for use by diluting to a final concentration if $6025 \mu \mathrm{g} / \mathrm{mL}$ in acetone. Additional information on the reagents is provided in the procedure in Appendix $B$.

Apparatus and materials: These are described in the procedure in Appendix A.

Analysis procedure: Prior to analjsis, $300 \mu \mathrm{L}$ of a sample residue (maintained at $4^{\circ} \mathrm{C}+1-2^{\circ} \mathrm{C}$ until ready for analysis) was transferred to an autosampler vial, and made up to $40 \mu \mathrm{g} / \mathrm{mL}$ with internal standards using the Supelco internal standards $m i x$. The GC/MS analysis followed the EPA CLP SOW $2 / 88$ conditions. The linearity of the instirument was verified from an initial 5-point calibration. The mass caltibration and tune check was performed and the instrument was calibrated using a single level continuing calibration. Each series of residue analyses was followed by analysis of a twotold dilution of the stock spike solution. 


\section{RESULTS}

In the following discussion, a description of the tabulated analysis data obtained in these studies is presented. Included are data from the prelimilary investigation and the initial and final development and validation studies.

\section{PRELIMINARY INVESTIGATION}

Figure 3 shows a chromatogram obtained with the $G C / f l a m e$ ionization detector of NPH. Figure 4 is a chromatogram of NPH eluted through a silica column using hexane, and Figure 5 is of NPH eluted through the same column with methylene chloride/hexane $(2: 3)$.

Figure 6 is a chromatogram of the hexane fraction eluted from a silica gel column containing NPH, base-neutral mix 2, and polycyclic aromatic hydrocarbon (PAH) mix. Figure 7 shows a chromatogrim of the fraction collected from the same column eluted with the methylene chloride/hexane mix. The calculated percent recoveries for selected compounds are shown in Table 1.

\section{INITIAL DEVELOPMENT AND VALIDATION STUDY}

Tables 2 through 5 show the results obtained from '/arious stages of the extraction, liquid/liquid partitions, and cleanup activities for the reagent blank, a method blank, a blank spike, and a spike duplicate utilizing the PivL cleanup. Blanks in tables indicate that no detectable quantity was found. The spikes for these studies include the complete semivolatile target compound list specified in EPA CLP-SOW $2 / 88$ and the method surrogate compounds. The steps at which aliquots for analysis were taken are presented in the flow diagram in Figure 1. The result from each step is expressed in terms of percent recovery. Step "Re-Step $1^{\prime \prime}$ is the re-analysis of the residue obtained from step one. The total recovery column shown is the sum of the recovery after the cleanup stage, in this case step P $3 B N$, and the acid partition stage, step P2A.

Tables 6 through 8 show the results obtained for the method blank, a blank spike, and a spike duplicate through the various procedural steps 
utilizing the EPA SW-846 method 3630 as the column cleanup. The total recovery is obtained by the sum of steps E2A, E2B, and E3N.

Tables 9 through 11 show the results obtained for the blank spike, a blank spike with NPH added, and a NPH-added duplicate emplaying the PNL cleanup. Additionally, step P3BN2 was used in this portion of the study. Step P3BN2 is the elution of the column with additional methylene chloride following step $P 3 B N$. The total recovery column is the sum of results obtained for steps P2A, P3BN, and P3BN2.

Tables 12 through 14 show the results obtained for the blank spike, a blank spike with NPH added, and a NPH-added duplicate employing the EPA cleanup. Additionally, step E3N2 was used in this portion of the study. Step E3N2 is the elution of the column with additional methylene chloride following step $E 3 N$. The total recovery column is the sum of results obtained for steps E2A, E2B, E3N, and E3N2.

Tables 15 through 23 show results for SST samples B110 and U110. The $\mathrm{NPH}$ in these samples are native to the samples. No additional NPH was spiked into the samples for this set of experiments.

Tables 15 through 17 show the results obtained for a sample from B110, a B110 spike, and a spike duplicate utilizing the PNL cleanup procedure.

Results for the un-spiked sample are expressed in terms of percent expected recovery had the sample been spiked. Ostensibly, the results for target compounds are comparable to units that are approximately 6 times $\mu \mathrm{g} / \mathrm{g}$. For example, a result of 100 percent as shown on the table is approximately a concentration of $600 \mu \mathrm{g} / \mathrm{g}$ in the sample. For $\mathrm{NPH}$, the level is 60 times $\mu \mathrm{g} / \mathrm{g}$. Therefore, 100 percent is approximately equal to a concentration of $6000 \mu \mathrm{g} / \mathrm{g}$ of NPH.

Tables 18 through 20 show the results obtained for a sample from U110, a U110 spike, and a spike duplicate employing the PNL cleanup procedure.

Tables 21 through 23 represent results obtained for a sample from B110, a B110 spike, and a spike duplicate utilizing the EPA cleanup procedure.

Table 24 summarizes the effect of the presence of NPH on target compound recoveries for the PNL and EPA cleanup methods. 


\section{FINAL DEVELOPMENT AND VALIDATION STUDY}

Table 25 shows the recoveries obtained from a blank and nine blank spike samples that have undergone the medium level solls extraction process. Table 26 summarizes these results by tabulating the mean recoveries from each of these analyses.

Tables 27 through 29 exhibits the recoveries obtained from the blank and the nine blank spikes to which the remainder of the cleanup process was applied as described previousiy in the procedures portion of this report. The total recovery is based on the results obtained for the acid fraction and the cleaned base-neutral fraction. Table 30 summarizes these results by tabulating the mean recoveries from each of these analyses. 


\section{DISCUSSION}

The results of the preliminary investigation, and the initial and final development and validation PNL cleanup studies, are interpreted in the following discussion. In addition, the method advisory control limits for the final PNL cleanup procedure is described.

\section{PRELIMINARY INVESTIGATION}

The results indicate that the NPH is eluted in a hexane elution of the silica gel column. Figure 4 shows a GC/FID chromatogram of NPH eluted with hexane. Figure 5 shows the same column eluted with methylene chloride/hexane $(2: 3)$. Approximately $90 \%$ of the NPH was recovered from the hexane fraction. The remaining $10 \%$ is shown to be recovered in the methylene chloride/hexane fraction.

The limited set of semivolatile analytes were seen to favor the methylene chloride/hexane fraction. Figure 6 is a chromatogram of the hexane fraction eluted from a silica gel column containing NPH, Supelco base-neutral mix 2, and Supelco PNA mix. While NPH is quite prevalent as expected, none of the analytes can be detected in the chromatogram. Figure 7 i11ustrates the recovery of the analytes and Table 1 provides the calculatec recoveries. The poor recoveries of the more polar, oxygen-containing compounds indicated a final elution of the column using a more polar solvent was in order. A more thorough method of developing the column cleanup at this point would have been to perform the cleanup using a solvent gradient and collect fractions or to employ high-performance liquid chromatography (HPLC) methods as an analog for development purposes. However, time and funding did not allow further development as the primary task. Any further development was limited to modifications made during the validation studies.

\section{INITIAL DEVELOPMENT AND VALIDATION STUDY}

The results of the work described in this document are summarized in Tables 2 through 23. The recoveries obtained from various stages of the extraction, liquid/liquid partitions, and cleanup activities for ali the 
experimental work are shown for both the PNL and EPA cleanup methods on blanks and samples, spiked blanks and samples, and blank splkes with and without NPH added. The steps at which aliquots for analysis were taken are presented in the flow diagram in Figure 1. The result from each step is shown to represent the successive losses expressed in terins of percent recovery from the initial standard. The results under column "Step 1" are the results from the soil extraction procedure. "Re-Step $l "$ is the re-analysis of the residue obtained from step one and is presented here to indicate the reproductbility of the analysis. Columns "P2BN" and "P2A" are results from the liquid/1iquid parti. tioning steps of the procedure and include losses from "Step 1" as well. Column "P3BN" shows the recovery obtained following the PNL column cleanup, and includes the losses from the partitioning step "P2BN" and the solls extraction stage "Step 1." The total recovery column shown is the sum of the recovery after the cleanup stage, in this case step P3BN, and the acid parti. tion stage, step P2A. The results for the acids are obviously not affected by the column cleanup step.

Results are simmartzed in Tables 2 through 5 for the PNL cleanup method for a reagent blank, a method blank, a blank spike, and a blank spike duplirate. These tables show that generally the substituted aniline compounds and 4-Chiorophenyl-phenylether exhibited the greatest losses during the solls extraction step. The dienes, substituted anilines, and phenols suffered the greatest losses during the partitioning steps. The results for the PNL cleanup step seem inconsistent. One would expect good results for the PAH compounds considering experience with the rather well established EPA cleanup procedure.

Based upon these observations, it is believed that the inconsistency arises from the column not adequately being flushed during the PNL cleanup. Columin void volume for the PNL cleanup is almost equal to the volume of the elution solvent. Consequently, there is little margin for error during the elution process. Much of the unexpected additional retention of analytes on the column, which results in low recoveries, is likely due to holdup on the column caused ty inadequate elution volume. This problem is seen in results described later in this discussion. 
The EPA cleanup method results for the method blank, a blank spike, and a blank spike duplicate are summartzed in Tables 6 through 8 . The total recovery values for this method are obtained from the sum of steps $22 A, E 2 B$, and E3N. In general, recovertes are fair for the phenols and PAH but not for the chlorinated compounds, neutrals, and phthalates. The partitioning steps and cleanup steps are relatively self consistent as are the total recoveries for the two blank spikes.

The results for the PNL cleanup for the blank spike, a blank spike with $\mathrm{NPH}$ added, and an NPH-added duplicate, along with results for step P3BN2, are summarized in Tables 9 through 11. Step P3BN2 is the elution of the column with the additional methylene chloride following step P3BN. This step was added because of the observed color in the column after step P3BN. The total recovery column is the sum of results obtained for steps P2A, P3BN, and P3BN2. Significant improvement in recovery was achieved for many of the compounds due to the added methylene chloride elution step as shown in the P3BN2 column in those tables. This improvement is directly related to the poor wash volume to column void volume ratio after step P3BN. The presence of the NPH also enhanced the recoveries as seen in the improved total recoveries of compounds for which no P3BN2 improvements were observed. Low recoveries for some of the bases, phthalates, and a few neutrals still exists. The general improvement in recoveries is also seen in the partitioning step. Improved column performance is likely a consequence of increased migration of analytes in the column due to the NPH before becoming stationary on the silica phase. Less methylene chloride mobile phase is then required to begin analyte elution from the column. The effect of the NPH on recoveries is summarized in Table 24. An average of $73 \%$ of the NPH was removed in these experiments.

The results for the EPA method, including step E3N2, for the blank spike, a blank spike with NPH added, and an NPH-added duplicate are summarized in Tables 12 through 14 . Step E3N2 is the elution of the column with methylene chloride following step E3N. Step E3N2 is not part of the EPA procedure, but was added because of the color remaining in the columr after step E $3 \mathrm{~N}$. The total recovery column is the sum of results obtained for steps E2A, E2B, $E 3 N$, and E3N2. The EPA method did not benefit as much as the PNL method from 
the additional methylene chloride elution step, because of the lower void volumes of the columns used in the EPA method. Recoveries were slightly improved for only Nitrobenzene, Nitrobenzene-d5, 2,6-Dinitrotoluene and $\mathrm{N}$ Nitrosodiphenylamine. The effect of the NPH upon recovery was generally negative as seen in Table 24. Only the Nitrobenzenes and $\mathrm{N}$-Nitrosodiphenylamine benefitted. In addition, the reproducibility of the duplicate results was worse. The average NPH removal for these duplicates was about $94 \%$.

Results are summarized in Tables 15 through 17 for SST sample B110, B110 spike, and the spike duplicate utilizing the PNL cleanup procedure. Results for the un-spiked sample are expressed in terms of percent expected recovery had the sample been spiked. These samples did not go through the P3BN2 step, and the results are parallel to the results shown in Table 5 for the blank spike duplicate. When comparing the results in Table 4 , inconsistencies are noted, especially for the Dichlorobenzenes. This is probably due to the small elution volume. As noted for the effect of NPH on the blank spikes, the NPH also generally enhances recoveries for the sample spikes. Although the reproducibility is poor for sample spike duplicates, the results are parallel. NPH removal was $82 \%$ for the unspiked sample and averaged $97 \%$ for the duplicates.

The results summarized in Tables 18 through 20 for SST sample U110, U110 spike, and the spike duplicate employing the PNL cleanup procedure also show inconsistencies. The chlorinated compounds are particularly inconsistent. Otherwise the results are similar to the blank spike results in Tables 4 and 5. Again, there is a general enhancement of recoveries that is attributable to the NPH. There was $99.8 \%$ removal of the NPH for the sample, and an average of $99.4 \%$ removal for the spiked sample duplicates.

The EPA cleanup results for SST sample B110, B110 spike, and spike duplicate are summarized in Tables 21 through 23. The duplicate sample spikes agree we11, and they parallel the EPA method results for the blank spikes with a few exceptions. In general, the compound recoveries were higher for the sample spikes than for the blank spikes, with the exception of the substituted anilines, Nitrobenzenes, and N-Nitrosodiphenylamine. The recovery differences, both positive and negative, are likely due to sample matrix effects and 
may have nothing to do with the presence of NPH. The NPH removal was $94 \%$ for the sample and averaged $88 \%$ for the sample spike duplicates.

Table 24 summarizes the effect of the NPH on recoveries in using the PNL and EPA cleanup methods. There is a clear improvement in recoveries of all but a very few compounds using the PNL method in the presence of NPH, while the opposite is true for the EPA method in the presence of NPH. As discussed previously, the improved PNL cleanup recoveries with NPH present is likely due to further migration of the analytes into the column, and hence the analytes are flushed more quickiy from the column. The EPA cleanup, by virtue of a substantially smaller column and proportionally larger solvent elution volumes, is not expected to exhibit the same effect. The reason for diminished recoveries for the EPA cleanup due to the presence of the NPH is not clear; the effect may be an inherent problem with the method as used. Explaining these discrepancies is outside the scope of this study.

\section{FINAL DEVELOPMENT AND VALIDATION STUDY}

The inconsistent results observed in the initial PNL cleanup method are thought to be primarily a consequence of the excessively large void volume of the cleanup column. Therefore, the use of the smaller, commercial pre-packed columns were tested in the final study. In addition, the low and inconsistent recoveries observed for some of the more polar and oxygen containing compounds in the initial work suggested the need for a final column elution with a more polar solvent. To this end, elution steps with methylene chloride/acetone (1:1) and acetone were added to the method.

In Table 25, the recoveries of the spike compounds and NPH are summarized for a blank and nine blank spikes with NPH, that have undergone a medium level soils extraction procedure without sample cleanup. Table 26 provides the mean recoveries and standard deviations STD $(N-1)$ for a 11 the compounds and $N P H$. In addition, the Mean Recovery $\pm 3 \times S T D(N-1)$ values for the surrogate and matrix spike compounds in the CLP SOW 2/88 method for the semivolatiles are included.

Recoveries for these compounds as well as for the NPH in a blank and the nine blank spikes with NPH after completion of the cleanup procedure are 
summarized in Tables 27 through 29. The mean recoveries and STD(N-1) values for the compounds and NPH are shown in Table 30 along with the mean recovery \pm $3 \times \operatorname{STD}(N-1)$ values for the surrogate and matrix spike compounds. Table 31 shows the effect of the cleanup procedure on the recoveries. The largest effects are noted for the diene and chlorinated compounds. The improvement for 2-Nitroaniline is probably an anomaly; there were considerable chromatography problems with the anilines in some of the samples. About $73 \%$ of the $\mathrm{NPH}$ was removed by the cleanup.

In comparing the results summarized in Table 31 (Mean After Cleanup column) and Tables 10 and 11 (Total Recovery columns), the improvement in performance using the "final" PNL method is striking: there are no inconsistencies, the reproducibility is better, and the recoveries are improved. There are no recoveries in the "teens" or less, as there were in the initial studies. Use of the more polar solvent for the final wash and the use of columns with the smaller void volume most certainly played an important role in this improvement.

\section{METHOD ADVISORY CONTROL LIMITS}

Application of this procedure to further SST analysis requires that meaningful advisory control limits are established. The final PNL cleanup method employing disposable cartridge columns is certainly the process of choice based upon cost, performance, and level of effort. To date, however, only the partition segment of the cleanup has been performed on actual waste samples while the cartridge cleanup has only been applied to blank spike samples. It can easily be inferred that the final PNL cartridge cleanup will perform well with actual samples. The reasons for this are several fold:

- Both the initial and final PNL cleanup methods used identical preparation processes; therefore, no differences exist prior to the actual column cleanup step.

- Data for blank spike samples, including the NPH interferant, from both cleanup methods can be compared to provide a good baseline understanding of process performance.

- Data from smaller columns have been obtained through assessment of the EPA procedure. Column capacity is not an issue. 
A quantitative derivation of the advisory control limits for SST wastes can be developed. As stated previously, the results for the samples up to the actual column cleanup step may be used as there was no difference in preparation to that point. If the recovery from the blank spike up to the column cleanup step employing the initial PNL cleanup method can be subtracted from the recovery of the final PNL cleanup method, the result will be the final cleanup step's effect on recovery apart from the influence of the previous steps. The resultant difference effect can then be subtracted from the recovery results for SST samples in the initial study prior to column cleanup. This may be expressed as follows:

Mean Recovery (SST sample) $=$ P2BN - (NMS -BN - B1ank BN) + Acid

$$
\begin{aligned}
\text { Standard Deviation }(N-1)= & {\left[(P 2 B N)^{2}+\left[\text { ABS }(\text { NMS }-B N)^{2}-(B 1 \text { ank BN })^{2}\right]+\right.} \\
& \left.(\text { Acid })^{2}\right]^{1 / 2}
\end{aligned}
$$

where: Mean Recovery is the percent recovery calculated for a given compound in SST samples.

Standard Deviation is calculated as $\mathrm{N}-1$

ABS is the absolute value

P2BN is the average recovery obtained from SST samples for a given compound through step P2BN.

NMS-BN is the average recovery obtained from blank spike + NPH s.amples for a given compound prior to the initial PNL column cleanup.

$B$ lank $B N$ is the average recovery obtained from blank spikes $+\mathrm{NPH}$ after final PNL column cleanup.

Acid is the average recovery obtained from SST samples for a given compound through the acid partitioning step.

The calculation neglects any matrix effect that may occur on the cleanup column, although this is believed to be small. Table 32 shows the data used for this calculation. Table 33 provides the precision, accuracy, and calculated advisory control limits for surrogate and spiking semivolatile compounds. 


\section{CONCLUSIONS}

As discussed previousiy, a PNL method has been developed and validated for use on samples that contain difficult levels of NPH. The sample is first extracted following PNL procedure PNL-ALO-120 or PNL-ALO-344. An aliquot of the extract is added to water, which is then made basic, and the base-neutral compounds are extracted into methylene chloride. The aqueous phase is made acidic, and the acidic compounds are extracted into methylene chloride. The base-neutral fraction is solvent exchanged into cyclohexane and eluted through a commercial pre-packed silica gel column. The NPH is eluted from the column with pentane, which is followed by elution of the base-neutrals with successively more polar solvents. This fraction and the acid fraction are concentrated and are ready for analysis.

The effectiveness of this cleanup method was demonstrated for al1 of the semivolatile target list compounds in the EPA CLP SOW 2/88. Both blanks and SST samples spiked with all target compounds and NPH were used in this development and validation study. The NPH removal averaged $94 \%$ for samples from SST B110 and $97 \%$ for samples from tank U110. The recoveries were good for most of the target compounds and satisfactory for all, although they were lower for the more troublesome compounds. Advisory quality control limits were established for the surrogate and matrix spike compounds. As more data are obtained using this cleanup method, required $Q C$ limits will be put in place, and a better definition of cleanup effectiveness can be established.

The NPH contamination was considered to be the overwhelming factor affecting GC/MS analysis. If other less conspicuous analytical difficulties exist, they may only now be detectable. For example, siloxanes have been detected in past analyses when large amounts of NPH were present. Following the cleanup procedure, siloxanes have still been detected during analysis of semivolatile residues. The detrimental contribution siloxanes may have, if any, has not been determined at this time.

Capillary gas chromatography as an analytical tool is well documented and offers high chromatographic resolution and sensitivity, and is specified in the current regulatory literature for semivolatile analysis. However, it 
lacks the ruggedness often required when characterizing complex wastes. Complimentary preparation and analyses employing more robust separation techniques such as liquid'chromatography should be developed and will certainly have broad application in waste tank characterization and should be explored in the near future. 


\section{REFERENCES}

EPA Contract Laboratory Program, Statement of Work for Orqanic Analys is, Multi-Media, Multi-Concentration, SOW No. 2:88.

EPA Contract Laboratory Program, Statement of Work for Organic Analys is, Multi-Media, Multi-Concentration, Document Number OLMO1.0, March 1990.

EPA Office of Solid Waste, Test Methods for Evaluating Solid Waste, SW-846, Third Edition, 11-86.

PNL-ALO-344, Procedure for Sample Preparattor, of Samples for GC/MS Analys is for Semivolatile Organic Compounds, Rev. 0, PNL Technical Procedure, 4-26-91.

PNL-AL0-345, GC/MS Analys is of Extractable Semivolatile Organic Compounds, Rev. 0, PNL Techntcal Procedure, 8-3-89. 


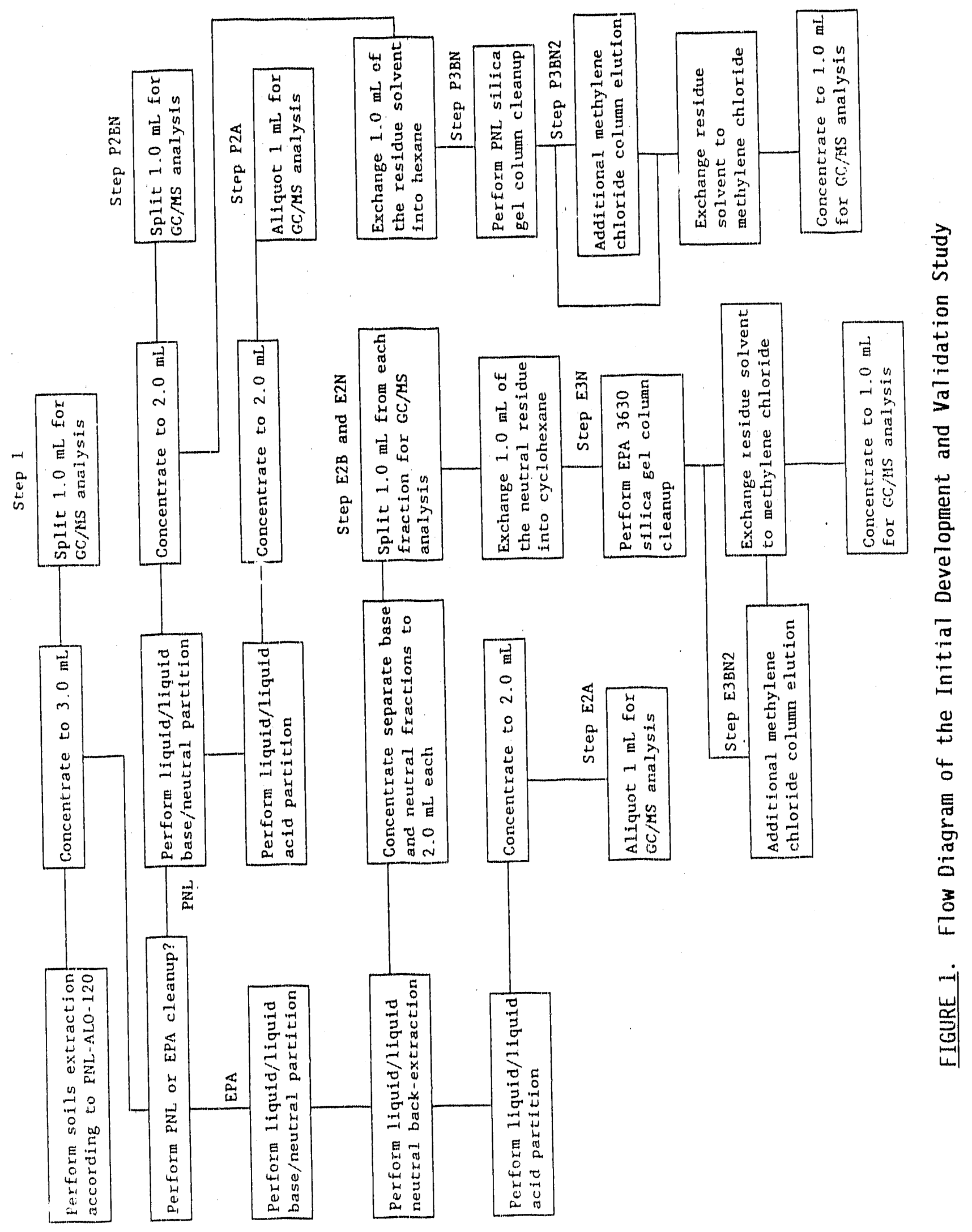




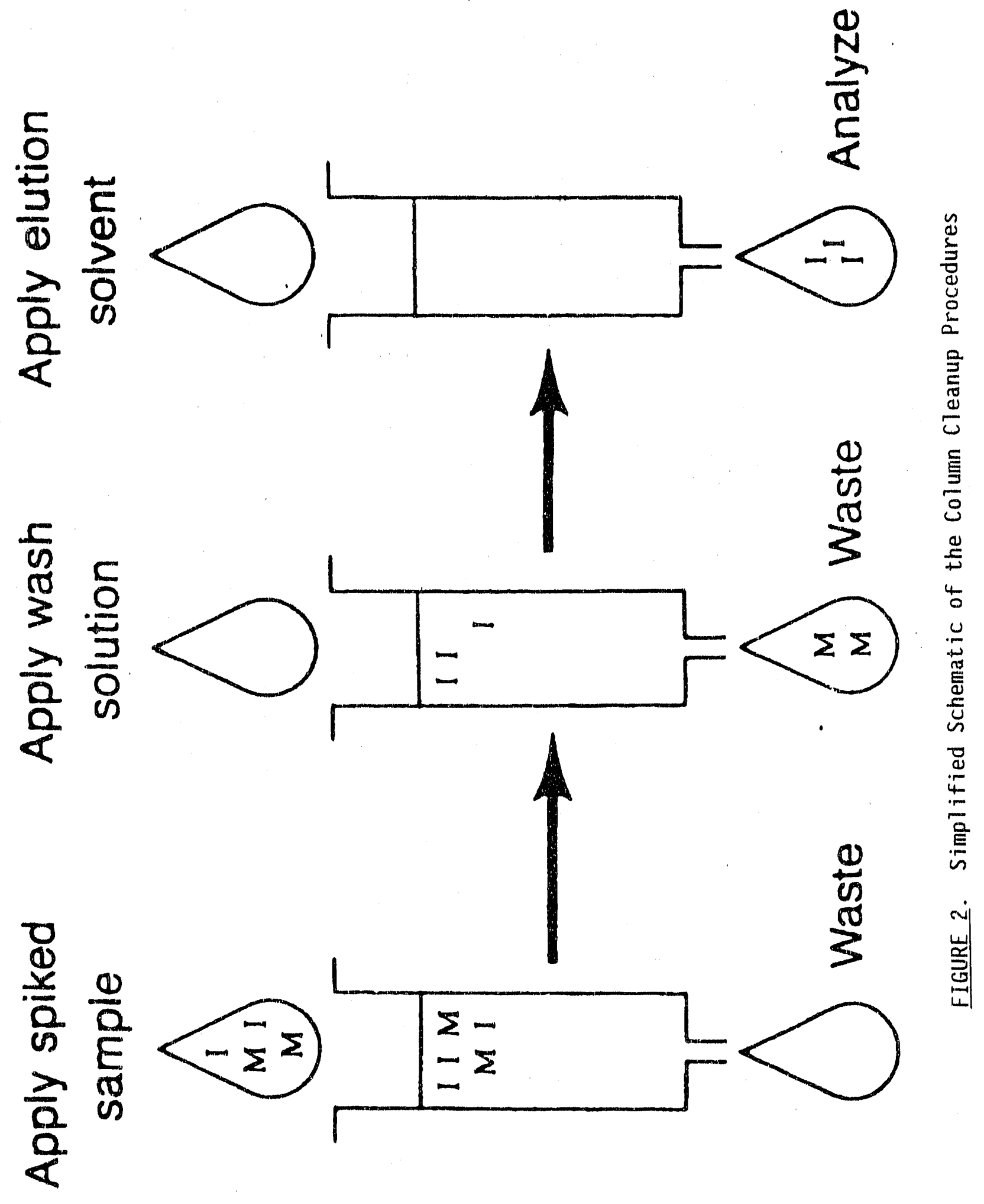




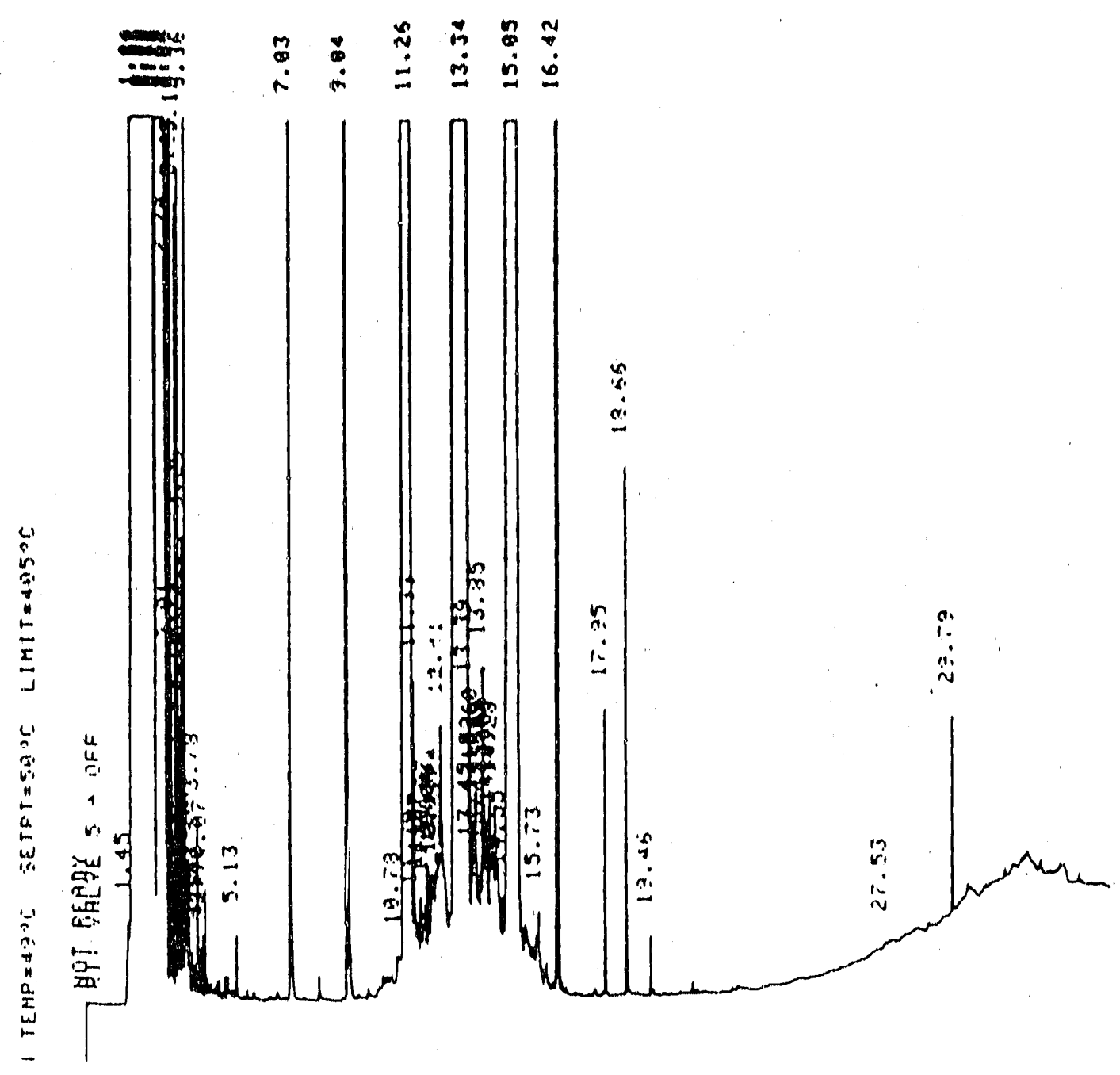

EIGURE 3. GC/FID Chromatogram of Normal Paraffin Hydrocarbon (NPH) 


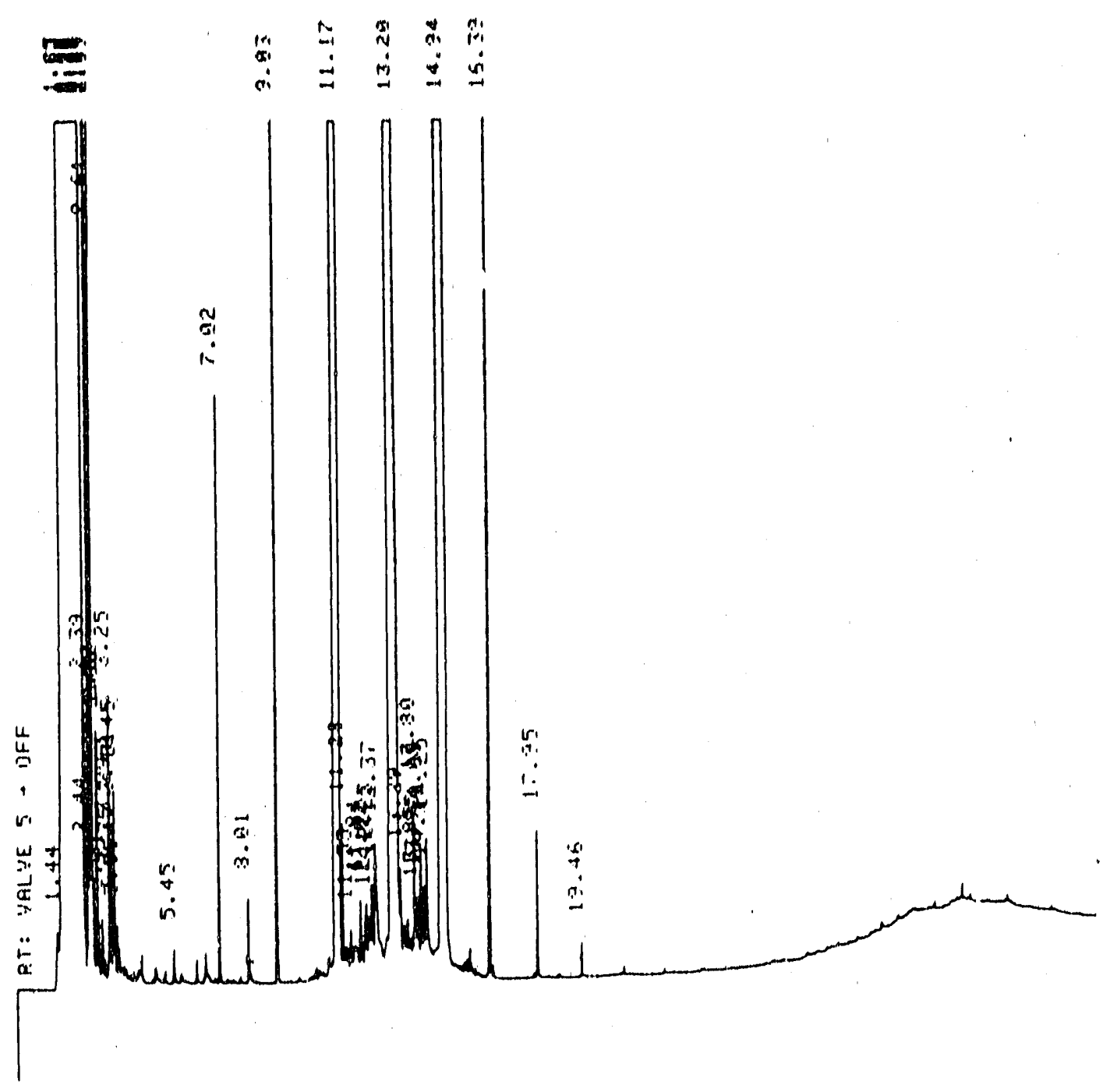

EIGURE 4. GC/FID Chromatogram of NPH Through Stlica Column Eluted with Hexane 


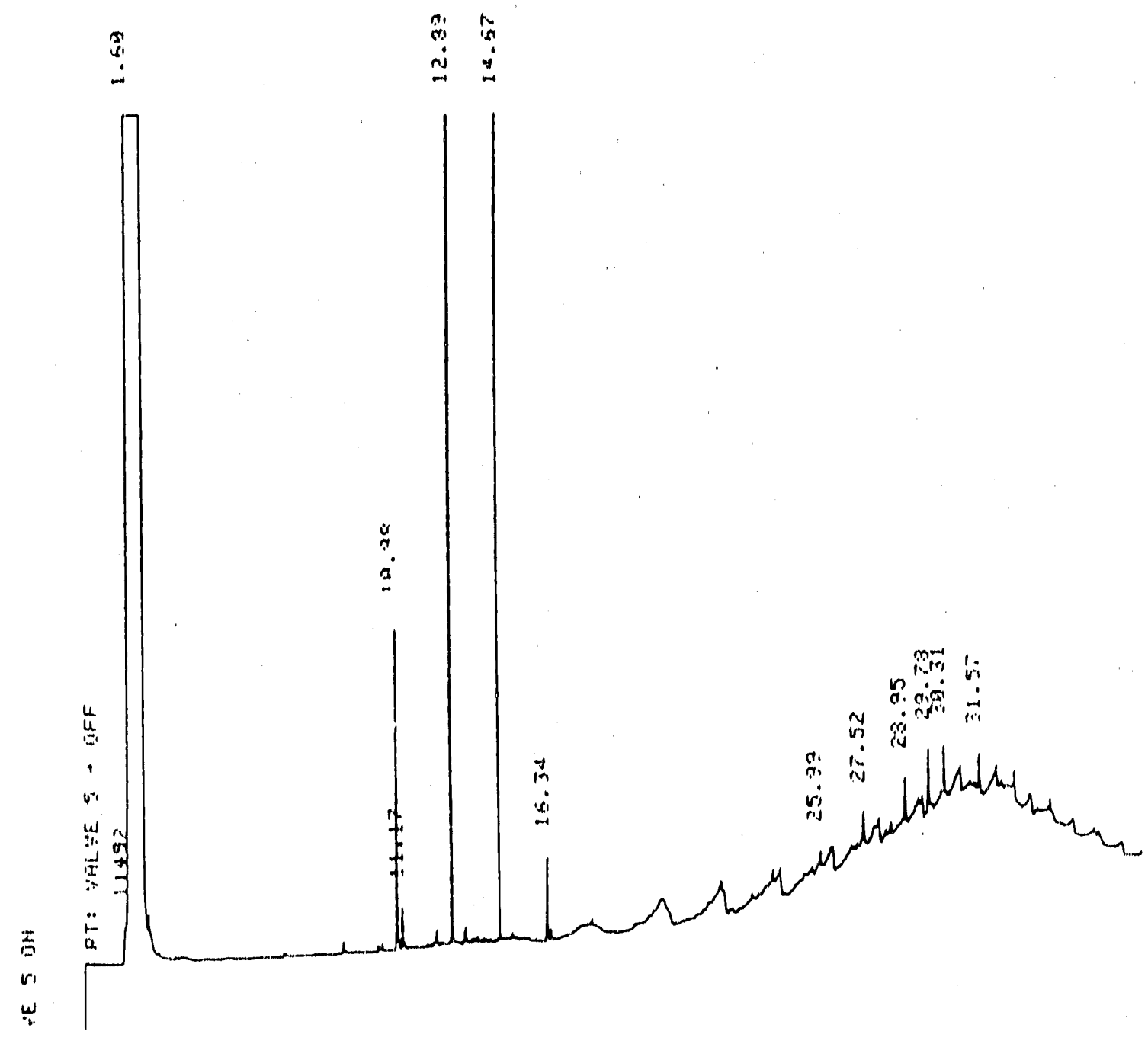

EIGURE 5. GC/FID Chromatogram of Methylene Chloride: Hexane Elution of NPH Following Hexane Elution 


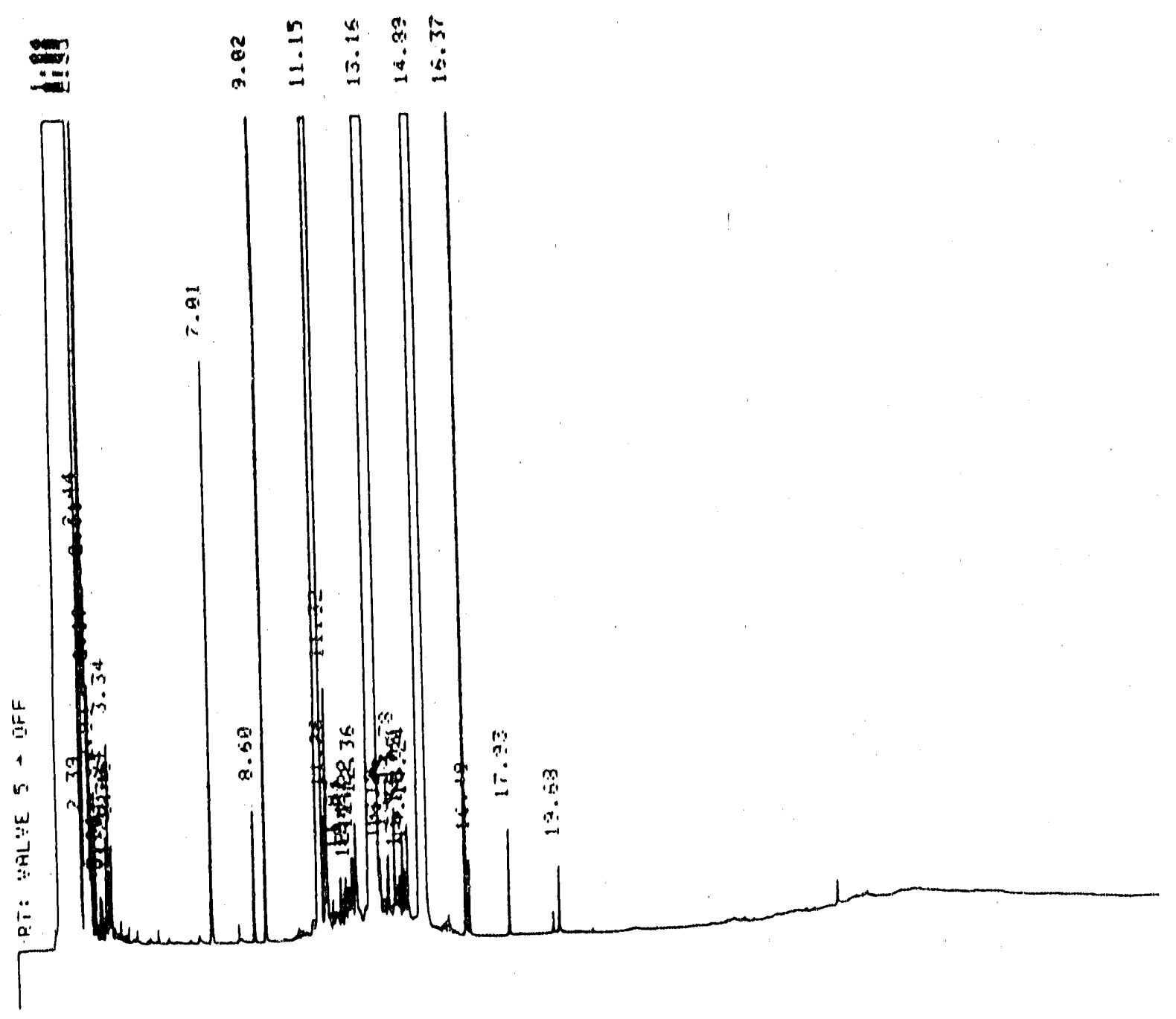

FIGURE 6. GC/FID Chromatogram of the Standard Mix Containing NPH, Base Neutral Mix 2, and AHs Through Silica Column Eluted with Hexane 


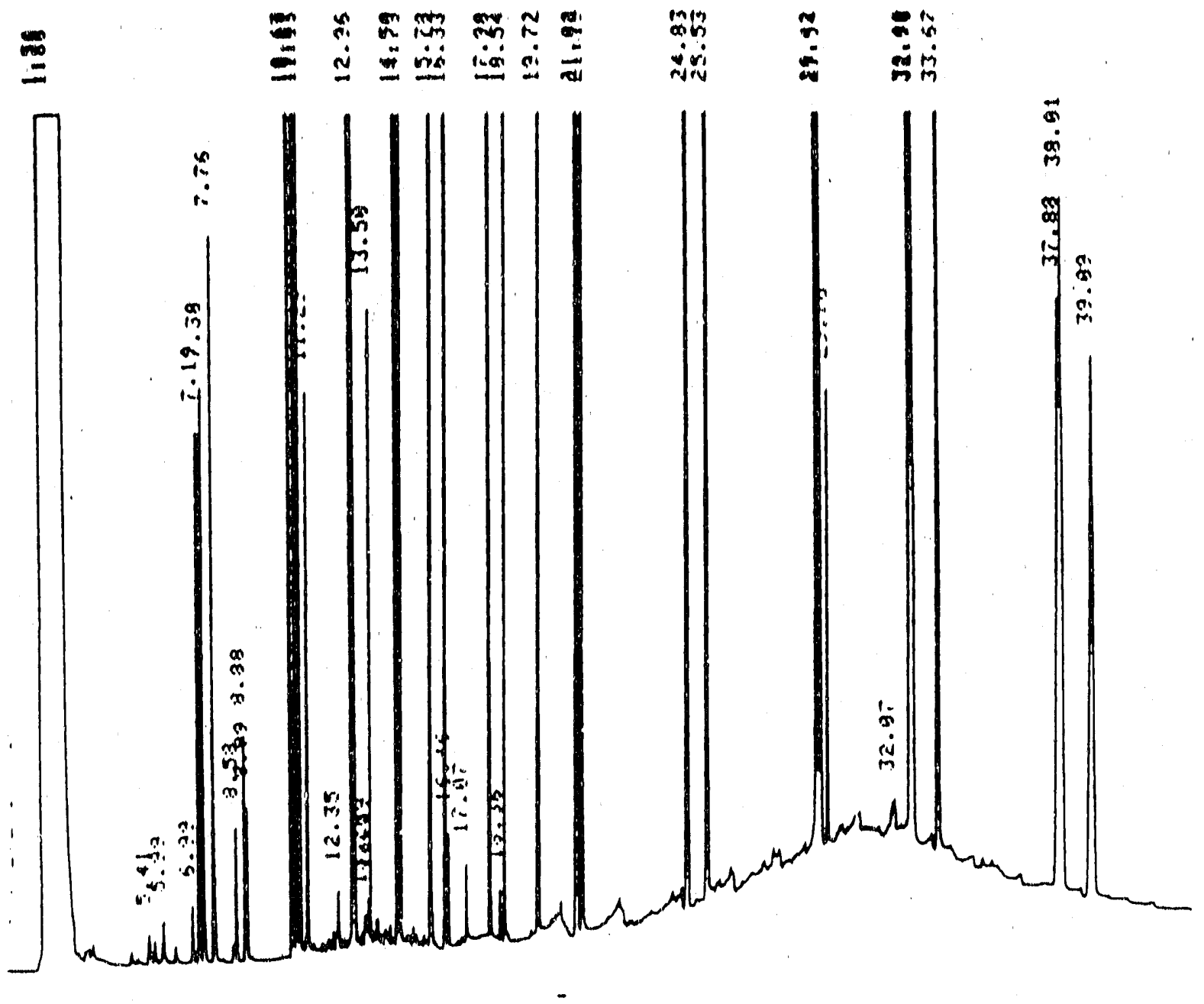

FIGURE 7. GC/FID Chromatogram of the Standard Mix Containing NPH, Base Neutral Mix 2, and PAHs Through Silica Column Eluted with Methylene Chloride: Hexane and then Methylene Chloride After Original Elution with Hexane 
IABLE 1. Calculated Percent Recoveries for Selected Compounds by $G C / F I D$, Preliminary Investigation

Sample: Prepared Standard Percent

No. Compound Name

Recovery

\begin{tabular}{|l|r|}
\hline 7) 1,3-Dichlorobenzene & 70 \\
\hline 25) 1,2,4-Trichlorobenzene & 87 \\
\hline 32) Hexachlorocyclopentadiene & 88 \\
\hline 46$)$ 2,4-Dinitrotoluene & 90 \\
\hline 48) 4-Chlorophenyl-phenylether & 0 \\
\hline 56$)$ Hexachlorobenzene & 95 \\
\hline 59) Anthracene & 91 \\
\hline 63$)$ Pyrene & 100 \\
\hline 55) Butylbenzylphthalate & 0 \\
\hline 68$)$ Chrysene & 100 \\
\hline 75) Indeno $(1,2,3-$ cd)pyrene & 84 \\
\hline
\end{tabular}



TABLE 2. Percent Recovery Results for Reagent Blank, Initial PNL
Cleanup Method

\begin{tabular}{|c|c|c|c|c|c|c|}
\hline $\begin{array}{l}\text { Sample: Battelle Reagent Blank } \\
\text { No. Compound Name }\end{array}$ & Step 1 & Re-Step 1 & P2BN & P2A & P3BN & $\begin{array}{r}\text { Total } \\
\text { Recovery }\end{array}$ \\
\hline 1) *1,4-Dichlorobenzene-d4 & 100.0 & 100.0 & 100.0 & 100.0 & 100.0 & 100.0 \\
\hline \multicolumn{7}{|l|}{ 2) \#2-Fluorophenol } \\
\hline \multicolumn{7}{|l|}{ 3) \#Phenol-d5 } \\
\hline \multicolumn{7}{|l|}{ 4) Phenol } \\
\hline \multicolumn{7}{|l|}{ 5) bls(2-Chloroethyl)ether } \\
\hline \multicolumn{7}{|l|}{ 6) 2-Chlorophenol } \\
\hline \multicolumn{7}{|l|}{ 7) 1,3-Dichiorobenzene } \\
\hline \multicolumn{7}{|l|}{ 8) 1,4-Dichlorobenzene } \\
\hline \multicolumn{7}{|l|}{ 9) Benzyl alcohol } \\
\hline \multicolumn{7}{|l|}{ 10) 1,2-Dichtorobenzene } \\
\hline \multicolumn{7}{|l|}{ 11) 2-Methylphenol } \\
\hline \multicolumn{7}{|l|}{ 12) bis (2-Chiorolsopropyl)ether } \\
\hline \multicolumn{7}{|l|}{ 13) 4-Methylphenol } \\
\hline \multicolumn{7}{|l|}{ 14) N-Nitroso-di-n-propylamine } \\
\hline \multicolumn{7}{|l|}{ 15) Hexachloroethane } \\
\hline 16) Naphthalen $\theta-d 8$ & 100.0 & 100.0 & 100.0 & 100.0 & 100.0 & 100.0 \\
\hline \multicolumn{7}{|l|}{ 17) \#Nitrobenzene-d5 } \\
\hline \multicolumn{7}{|l|}{ 18) Nitrobenzeno } \\
\hline \multicolumn{7}{|l|}{ 19) Isophorone } \\
\hline \multirow{2}{*}{\multicolumn{7}{|c|}{$\begin{array}{l}\text { 20) 2-Nitrophenol } \\
\text { 21) 2,4-Dimethylphenol }\end{array}$}} \\
\hline \multirow{2}{*}{\multicolumn{5}{|c|}{ 21) 2,4-Dimethylphenol }} & & \\
\hline \multirow{2}{*}{\multicolumn{7}{|c|}{ 22) Benzoic acid }} \\
\hline & & & \multicolumn{4}{|c|}{ 23) bis (2-Chloroethoxy)methane } \\
\hline \multirow{2}{*}{\multicolumn{7}{|c|}{ 24) 2,4-Dichlorophenol }} \\
\hline & & \multicolumn{5}{|c|}{ 25) 1,2,4-Trichlorobenzene } \\
\hline \multicolumn{7}{|l|}{ 26) Naphthalene } \\
\hline 27) 4-Chloroanilline & & & & & & \\
\hline 28) Hexachlorobutadiene & & & & & & \\
\hline 29) 4-Chloro-3-methylphenol & & & & & & \\
\hline 30) 2-Methylnaphthalene & & & & & & \\
\hline 31) Acenaphthene-d10 & 100.0 & $100 . \overline{0}$ & 100.0 & 100.0 & 100.0 & 100.0 \\
\hline 32) Hexachlorocyclopentadiene & & & & & & \\
\hline 33) 2,4,6-Trichlorophenol & & & & & & \\
\hline 34) 2,4,5-Trichlorophenol & & & & & & \\
\hline 35) 2-Chloronaphthalene & & & & & & \\
\hline 36) \#2-Fluorobliphenyl & & & & & & \\
\hline 37) 2-Nitroanilline & & & & & & \\
\hline 38) Dimethylphthalate & & & & & & \\
\hline 39) Acenaphthylene & & & & & & \\
\hline 40) 2,6-Dinitrotoluene & & & & & & \\
\hline 41) 3-Nitroaniline & & & & & & \\
\hline
\end{tabular}


TABLE 2. (contd)

Sample: Battelle Reagent Blank

No. Compound Name

42) Acenaphthene

43) 2,4-Dinitrophenol

44) 4-Nitrophenol

45) Dibonzofuran

46) 2,4-Dinitrotoluene

47) Diethylphthalato

48) 4-Chlor--henyl-phenylether

49) Fluorene

50) 4-Nitroanilline

51) *Phenanthrene-d10

52) 4,6-Dinitro-2-methylpheno

53) $\mathrm{N}$-Nitrosodiphenylamine

54) \#2,4,6-Tribromophenol

55) 4-Bromophenyl-phenylether

56) Hexachlorobenzene

57) Pentachlorophenol

58) Phenanthrene

59) Anthracene

60) DI-n-butylphthalate

61) Fluoranthene

62) *Chrysene-d12

63) Pyrene

64) \#Terphenyl-d14

65) Butylbenzylphthalate

66) 3,3'-Dichlorobenzidine

67) Benzo(a)anthracene

68) Chrysen $\theta$

69) Bis(2-Ethylhexyl)ph thalate

70) "Perylene-d12

71) DI-n-octylphthalate

72) Benzo(b)fluoranthene

73) Benzo (k) fluoranthene

74) Benzo(a)pyrene

75) Indeno (1,2,3-cd)pyrene

76) Dibenz $(a, h)$ anthracene

77) Benzo $(g, h, i)$ peryiene

NPH (Paraffin Hydrocarbon, C12-C14)

* Compound is an Internal Standard

\# Compound is a Surrogate Spike 


\section{TABLE 3. Percent Recovery Results for Method Blank, Initial}

\section{PNL Cleanup Method}

\begin{tabular}{|c|c|c|c|c|c|c|}
\hline $\begin{array}{l}\text { Sample: Battelle Method Blank } \\
\text { No. Compound Name }\end{array}$ & Step 1 & $R \theta-S t e p 1$ & P28N & P2A & P3BN & $\begin{array}{r}\text { Total } \\
\text { Recovery }\end{array}$ \\
\hline 1) ${ }^{4}, 4$-Dlchlorobenzene-d4 & 100.0 & 100.0 & 100.0 & 100.0 & 100.0 & 100.0 \\
\hline 2) \#2-Fluorophenol & 87.4 & 87.8 & 0.3 & 27.4 & & 27.4 \\
\hline 3) \#Phenol-d5 & 94.4 & 95.7 & 4.2 & 44.1 & & 44.1 \\
\hline 4) Phenol & & & & & & \\
\hline 5) bls(2-Chloroethyl)ether & & & & & & \\
\hline 6) 2-Chlorophenol & & & & & & \\
\hline 7) 1,3-Dichlorobanzene & & & & & & \\
\hline 8) 1,4-Dlchlorobenzene & & & & & & \\
\hline 9) Benzyl alcohol & & & & & & \\
\hline 10) 1,2-Dichlorobenzene & & & & & & \\
\hline 11) 2-Mesthylphenol & & & & & & \\
\hline 12) bls(2-Chloroisopropyl)ether & & & & & & \\
\hline 13) 4-Methylphenol & & & & & & \\
\hline 14) $N$-Nitroso-di-n-propylamine & & & & & & \\
\hline 15) Hexachloroethane & & & & & & \\
\hline 16) *Naphthaleng-d8 & 100.0 & 100.0 & 100.0 & 100.0 & 100.0 & 100.0 \\
\hline 17) \#Nitrobenzene-d5 & 78.4 & 79.5 & 65.4 & 0.2 & & 0.2 \\
\hline 18) Nitrobenzene & & & & & & \\
\hline 19) Isophorone & & & & & & \\
\hline 20) 2-Nitrophenol & & & & & & \\
\hline 21) 2,4-Dimethylphenol & & & & & & \\
\hline 22) Benzoic acid & & & & & & \\
\hline 23) bis(2-Chloroethoxy)methane & & & & & & \\
\hline 24) 2,4-Dlchlorophenol & & & & & & \\
\hline 25) 1,2,4-Trichlorobenzene & & & & & & \\
\hline 26) Naphthalene & & & & & & \\
\hline 27) 4-Chloroaniline & & & & & & \\
\hline 28) Hexachlorobutadiene & & & & & & \\
\hline 29) 4-Chioro-3-methylphenol & & & & & & \\
\hline 30) 2-Methylnaphthalene & & & & & & \\
\hline 31) *Acenaphthene-d10 & 100.0 & 100.0 & 100.0 & 100.0 & 100.0 & 100.0 \\
\hline 32) Hexachlorocyclopentadlene & & & & & & \\
\hline 33) 2,4,6-Trichlorophenol & & & & & & \\
\hline 34) 2,4,5-Trichlorophenol & & & & & & \\
\hline 35) 2-Chloronaphthalene & & & & & & \\
\hline 36) \#2-Fluorobiphenyl & 77.4 & 84.5 & 68.8 & 0.7 & 46.3 & 46.9 \\
\hline 37) 2-Nitroaniline & & & & & & \\
\hline 38) Dimethy/phthalate & & & & & & \\
\hline 39) Acenaphthylene & & & & & & \\
\hline 40) 2,6-Dinitrotoluene & & & & & & \\
\hline 41) 3-Nitroaniline & & & & & & \\
\hline
\end{tabular}


TABLE 3. (contd)

\begin{tabular}{|c|c|c|c|c|c|c|}
\hline $\begin{array}{l}\text { Sample: Battelle Method Blank } \\
\text { No. Compound Name }\end{array}$ & Step 1 & Pe-Step 1 & P2BN & P2A & P.3BN & $\begin{array}{r}\text { Total } \\
\text { Recovery }\end{array}$ \\
\hline 42) Acenaphthene & & & & & & \\
\hline 43) 2,4-Dinltrophenol & & & & & & \\
\hline 44) 4-Nitrophenol & & & & & & \\
\hline 45) Dibenzofuran & & & & & & \\
\hline 46) 2,4-Dinitrotoluene & & & & & & \\
\hline 47) Dlothylphthalato & & & & & & \\
\hline 48) 4-Chlorophenyl-phenylether & & & & & & \\
\hline 49) Fluorene & & & & & & \\
\hline 50) 4-Nitroaniling & & & & & & \\
\hline 51) *Phenanthrene-d10 & 100.0 & 100.0 & 100.0 & 100.0 & 100.0 & 100.0 \\
\hline 52) 4,6-Dinitro-2-methylphenol & & & & & & \\
\hline 53) $\mathrm{N}$-Nitrosodiphenylamine & & & & & & \\
\hline 54) \#2,4,6-Tribromophenol & 107.1 & 114.0 & & 15.3 & & 15.3 \\
\hline 55) 4-Bromophenyl-phenylether & & & & & & \\
\hline 56) Hexachlorobenzene & & & & & & \\
\hline 57) Pentachlorophenol & & & & & & \\
\hline 58) Phenanthrene & & & & & & \\
\hline 59) Anthracent & & & & & & \\
\hline 60) Dl-n-butylphthalate & & & & & & \\
\hline 61) Fluoranthene & & & & & & \\
\hline 62$)^{\star} \mathrm{Chrysen} \theta-\mathrm{d} 12$ & 100,0 & 100.0 & 100.0 & 100.0 & 100.0 & 100.0 \\
\hline 63) Pyrenio & & & & & & \\
\hline 64) \#Terphenyl-d14 & 87.3 & 97.6 & 73.0 & 0.8 & 66.6 & 67.4 \\
\hline 65) Butylbenzylphthalate & & & & & & \\
\hline 66) 3,3-Dichlorobenziding & & & & & & \\
\hline 67) Benzo(a)anthracene & & & & & & \\
\hline 68) Chrysene & & & & & & \\
\hline 69) Bis(2-Ethylhexyl)phthalate & & & & & & \\
\hline 70) *Perylene-d12 & 100.0 & 100.0 & 100.0 & 100.0 & 100.0 & 100.0 \\
\hline 71) DI-n-octylphthalate & & & & & & \\
\hline 72) Benzo(b)fluoranthene & & & & & & \\
\hline 73) Benzo(k)fluoranthene & & & & & & \\
\hline 74) Benzo(a)pyrene & & & & & & \\
\hline 75) Indeno $(1,2,3-c d)$ pyrene & & & & & & \\
\hline 76) Dibenz $(a, h)$ anthracene & & & & & & \\
\hline 77) Benzo $(g, n, 1)$ perylene & & & & & & \\
\hline NPH (Paraffin Hydrocarbon, C12-C14) & & & & & & \\
\hline
\end{tabular}

* Compound is an Internal Standard

\# Compound is a Surrogate Spike 
TABLE 4. Percent Recovery Results for Blank Spike, Initial PNL Cleanup Method

\begin{tabular}{|c|c|c|c|c|c|c|}
\hline $\begin{array}{l}\text { Sample: PNL Blank Splke } \\
\text { No. Compound Name }\end{array}$ & Step 1 & Re-Step 1 & P2BN & P2A & P3BN & $\begin{array}{r}\text { Total } \\
\text { Recovery }\end{array}$ \\
\hline 1) *1,4-Dichlorobenzene-d4 & 100.0 & 100.0 & 100.0 & 100.0 & 100.0 & 100.0 \\
\hline 2) \#2-Fluorophenol & 98.6 & 93.3 & 0.1 & 37.3 & & 37,3 \\
\hline 3) \#Phenol-d5 & 95.2 & 91.0 & 1.9 & 40.8 & & 40.8 \\
\hline 4) Phenoi & 94.8 & 90.6 & 1.9 & 35.3 & & 35.3 \\
\hline 5) bis(2-Chloroethyl)ether & 79.7 & 76.6 & 62.6 & 0.2 & & 0.2 \\
\hline 6) 2-Chlorophenol & 92.3 & 88.3 & 0.4 & 36.7 & & 36.7 \\
\hline 1) 1,3-Dlchlorobenzene & 88.3 & 85.9 & 63.4 & 0.1 & 2.3 & 2.4 \\
\hline 8) 1,4-Dichlorobenzene & 88.9 & 87.6 & 61.4 & 0.1 & 2.3 & 2.4 \\
\hline 9) Benzyl alcohol & 92.3 & 85.1 & 62.4 & 0.5 & & 0.5 \\
\hline 10) 1,2-Dlchlorobenzene & 90.6 & 82.6 & 58.2 & 0.1 & 2.2 & 2.3 \\
\hline 11) 2-Methylphenol & 87.2 & 82.8 & 10.8 & 32.3 & & 32.3 \\
\hline 12) bls(2-Chlorolsopropyl)ether & 101,6 & 93.8 & 72.1 & 0.2 & & 0.2 \\
\hline 13) 4-Methylphenol & 93.8 & 88.3 & 7.5 & 34.0 & & 34.0 \\
\hline 14) $N-N \mid$ troso-di -n-propylamine & 96.9 & 90.8 & 70.3 & & & \\
\hline 15) Hexachloroethane & 89.2 & 85.0 & 59.2 & 0.1 & 1.4 & 1.4 \\
\hline 16) Naphthalene-d8 & 100.0 & 100.0 & 100.0 & 100.0 & 100.0 & 100.0 \\
\hline 17) \#Nitrobenzene-d5 & 82.7 & 83.9 & 66.3 & 0.1 & & 0.1 \\
\hline 18) Nitrobenzene & 88.3 & 85.3 & 63.5 & 0.1 & & 0.1 \\
\hline 19) Isophorone & 89.9 & 84.1 & 61.2 & 0.2 & & 0.2 \\
\hline 20) 2-Nitrophenol & 89.7 & 88.4 & & 39.6 & & 39.6 \\
\hline 21) 2,4--Dimethylphenol & 85.8 & 84.7 & 33.8 & 16.4 & & 16.4 \\
\hline 22) Benzolc acld & 89.5 & 85.1 & & 25.1 & & 25.1 \\
\hline 23) bis(2-Chloroethoxy)methane & 91.0 & 90.6 & 66.5 & 0.2 & & 0.2 \\
\hline 24) 2,4-Dichlorophenol & 89.2 & 89.0 & 0.3 & 43.2 & & 43.2 \\
\hline 25) 1,2,4-Trichlorobenzene & 83.0 & 83.0 & 62.6 & 0.2 & 2.9 & 3.1 \\
\hline 26) Naphthalene & 84.2 & 86.7 & 62.1 & 0.6 & 3.3 & 3.9 \\
\hline 27) 4-Chloroanilline & 44.5 & 31.7 & 18.7 & 0.0 & & 0.0 \\
\hline 28) Hexachlorobutadiene & 82.4 & 83.7 & 58.9 & 0.2 & 2.3 & 2.4 \\
\hline 29) 4-Chloro-3-methylphenol & 94.3 & 91.1 & 6.9 & 39.4 & & 39.4 \\
\hline 30) 2-Methylnaphthalene & 86.0 & 87.7 & 64.3 & 0.3 & 3.1 & 3.4 \\
\hline 31) *Acenaphthene-d10 & 100.0 & 100.0 & 100.0 & 100.0 & 100.0 & 100.0 \\
\hline 32) Hexachlorocyclaperitadiene & 86.8 & 85.1 & 49.4 & & 0.2 & 0.2 \\
\hline 3,3) 2,4,6-Trichlorophenol & 97.2 & 97.8 & & 45.3 & & 45.3 \\
\hline 34) 2,4,5-Trlohlorophenol & 87.4 & 90.5 & & 43.4 & & 43.4 \\
\hline 35) 2-Chloronaphthalene & 84.8 & 87.0 & 67.3 & 0.2 & 2.9 & 3.2 \\
\hline 36) \#2-Fluoroblphenyl & 83.4 & 88.9 & 69.7 & 0.3 & 3.5 & 3.7 \\
\hline 37) 2-Nitroanilline & 98.3 & 92.9 & 76.2 & 0.1 & & 0.1 \\
\hline 38) Dimethylphthalate & 9.4 .7 & 92.9 & 71.9 & 0.3 & & 0.3 \\
\hline 39) Acenaphthylene & 88.1 & 90.7 & 67.8 & 0.3 & 3.1 & 3.4 \\
\hline 40) $2,6-$ Dinitrotoluene & 94.9 & 89.1 & 73.2 & & & \\
\hline 41) 3-Nitroaniline & 96.2 & 64.0 & 37.8 & & & \\
\hline
\end{tabular}


TABLE 4. (contd)

\begin{tabular}{|c|c|c|c|c|c|c|}
\hline $\begin{array}{l}\text { Sample: PNL. Blank Splke } \\
\text { No. Compound Name }\end{array}$ & Step 1 & Re-Step 1 & $\mathrm{P} 2 \mathrm{BN}$ & P2A & P3BN & $\begin{array}{r}\text { Total } \\
\text { Recovery }\end{array}$ \\
\hline 42) Acenaphthene & 92.4 & 91.9 & 72.8 & 0.3 & 3.7 & 4.0 \\
\hline 43) 2,4-DInitrophenol & 106,8 & 93.7 & & 25.7 & & 26.7 \\
\hline 44) 4-Nitrophenol & 106.2 & 97.5 & & 40.9 & & 40.9 \\
\hline 45) Dlbenzofuran & 90,3 & 92.0 & 71.8 & 0.3 & 3.7 & 4.0 \\
\hline 46) 2,4-Dinitrotoluene & 93.8 & 94.0 & 72.8 & 0.3 & & 0.3 \\
\hline 47) Dlettylphthalate & 99.0 & 95.5 & 79.3 & 0.4 & 0.1 & 0.5 \\
\hline 48) 4-Chlorophenyl-phenylethar & 58.6 & 57.4 & 44.7 & 0.2 & 2.3 & 2.5 \\
\hline 49) Fluorene & 91.1 & 91.1 & 72.7 & 0.3 & 3.6 & 3.9 \\
\hline 50) 4-Nitroanlline & 63.0 & 86.8 & 62.4 & 0.1 & & 0.1 \\
\hline 51) *Phenanthrene-d10 & 100.0 & 100.0 & 100.0 & 100.0 & 100.0 & 100.0 \\
\hline 52) 4,6-Dinilto-2-methylphenol & 100.3 & 96.2 & & 37.9 & & 37.9 \\
\hline 53) $\mathrm{N}$-Nitrosodiphenylamine & 101.4 & 107.6 & 71.8 & 0.2 & & 0.2 \\
\hline 54) \#2,4,6-Tribromophenol & 98.5 & 105.2 & & 45.7 & & 45.7 \\
\hline 55) 4-Bromophenyl-phenylether & 88.2 & 91.4 & 66.4 & 0.3 & 3.6 & 3.9 \\
\hline 56) Hexachlorobenzene & 92.8 & 93.8 & 69.3 & 0.4 & 3.9 & 4.2 \\
\hline 57) Pentachlorophenol & 96.0 & 102.4 & & 45.8 & & 45.8 \\
\hline 58) Phenanthrene & 92.4 & 93.1 & 72.9 & 0.4 & 4.6 & 5.0 \\
\hline 59) Anthracene & 91.1 & 92.1 & 68.0 & 0.3 & 4.0 & 4.3 \\
\hline 60) DI-n-butylphthalate & 92.3 & 93.0 & 72.6 & 0.5 & 0.3 & 0.8 \\
\hline 61) Fluoranthene & 91.8 & 90.0 & 68.5 & 0.4 & 5.1 & 5.5 \\
\hline 62) *Chrysene-d12 & 100.0 & 100.0 & 100.0 & 100.0 & 100.0 & 100.0 \\
\hline 63) Pyrene & 94.9 & 88.7 & 72.0 & 0.4 & 4.8 & 5.1 \\
\hline 64) \#Terphenyl-d14 & 81.2 & 81.9 & 67.9 & 0.4 & 5.6 & 6.0 \\
\hline 65$)$ Butylbenzylphthalate & 95.9 & 88.6 & 75.8 & 0.4 & 0.2 & 0.6 \\
\hline 66) $3,3^{\prime}-$ Dlchlorobenzldine & 100.0 & 71.4 & 73.5 & 0.1 & & 0.1 \\
\hline 67) Benzo(a)anthracene & 96.2 & 90.5 & 76.3 & 0.5 & 5.6 & 6.1 \\
\hline 68) Chrysene & 88.7 & 84.4 & 75.2 & 0.5 & 6.2 & 6.7 \\
\hline 69) Bls(2-Ethylhexyl)phthalate & 88.4 & 87.4 & 72.0 & 0.4 & & 0.4 \\
\hline 70) "Perylene-d12 & 100.0 & 100.0 & 100.0 & 100.0 & 100.0 & 100.0 \\
\hline 71) DI-n-octylphthalate & 84.0 & 93.7 & 69.5 & 0.3 & 0.1 & 0.4 \\
\hline 72) Benzo(b)fluoranthene & 96.4 & 98.0 & 75.3 & 0.4 & 4.5 & 4.9 \\
\hline 73) Benzo (k) fluoranthene & 70.7 & 89.9 & 69,3 & 0.5 & 4.7 & 5.2 \\
\hline 74) Benzo(a)pyrene & 91.3 & 96.4 & 70.0 & 0.4 & 4.1 & 4.5 \\
\hline 75$)$ Indeno $(1,2,3-$ cd)pyrene & 107.4 & 104.2 & 76.3 & 0.3 & 3.3 & 3.6 \\
\hline 76) Dibenz (a,h)anthracene & 85.9 & 92.4 & 68.2 & 0.3 & 2.8 & 3.1 \\
\hline 77) Benzo $(g, h, 1)$ perylene & 99.7 & 98.1 & 78.6 & 0.4 & 2.9 & 3.3 \\
\hline
\end{tabular}

\begin{tabular}{|l|l|l|l|l|}
\hline NPH (Paraffln Hydrocarbon, C12-C14) & & & & \\
\hline
\end{tabular}

* Compound is an Intemal Standard

\# Compound is a Surrogate Splke 
IABLE 5. Percent Recovery Results for Blank Spike Duplicate, Initial PNL Cleanup Method

\begin{tabular}{|c|c|c|c|c|c|c|}
\hline $\begin{array}{l}\text { Sample: PNL Blank Splke Dupllcate } \\
\text { No. Compound Name }\end{array}$ & Step 1 & Be-Step 1 & P2BN & P2A & P3BN & $\begin{array}{r}\text { Total } \\
\text { Recovery }\end{array}$ \\
\hline 1) ${ }^{* 1,4-D i c h l o r o b e n z e n e-d 4}$ & 100.0 & 100.0 & 100.0 & 100.0 & 100.0 & 100.0 \\
\hline 2) \#2-Fluorophenol & 107.3 & 104.9 & 0.2 & 39.4 & & 39.4 \\
\hline 3) \#Phenol-d5 & 101.4 & 102.0 & 1.9 & 42.5 & & 42.5 \\
\hline 4) Phenol & 101.6 & 103.6 & 2.0 & 39.0 & & 39.0 \\
\hline 5) bls(2-Chloroethyl)ether & 85.1 & 87.4 & 64.8 & 0.1 & & 0.1 \\
\hline 6) 2-Chlorophenol & 98.7 & 98.4 & 0.6 & 40.7 & & 40.7 \\
\hline 7) 1,3-0ichlorobenzene & 100.7 & 97.2 & 65.4 & 0.1 & 34,3 & 34.4 \\
\hline 8) 1,4-Dlchlorobenzene & 91.5 & 96.4 & 63.6 & 0.1 & 37.1 & 37.2 \\
\hline 9) Benzyl alcohol & 100.2 & 96.5 & 66.5 & & & \\
\hline 10) 1,2-Dlchlorobenzene & 95.6 & 91.4 & 62.4 & 0.1 & 36.5 & 36.6 \\
\hline 11) 2 -Methylphenol & 93.1 & 94.8 & 12.1 & 35.8 & & 35.8 \\
\hline 12) bls(2-Chlorolsopropyl)ether & 108.5 & 103,9 & 78.2 & 0.1 & & 0.1 \\
\hline 13) 4-Methylphenol & 103.2 & 100.1 & 9.0 & 37.1 & & 37.1 \\
\hline 14) N-Nitroso-di-n-propylamine & 102.8 & 99.7 & 75.2 & 0.1 & & 0.1 \\
\hline 15) Hexachloroethane & 95.0 & 96.1 & 62.7 & 0.0 & 27.3 & 27,4 \\
\hline 16) *Naphthalen $\theta-d 8$ & 100.0 & 100.0 & 100.0 & 100.0 & 100.0 & 100.0 \\
\hline 17) \#Nitrobenzene-d5 & 89.0 & 95.5 & 69.8 & 0.1 & & 0.1 \\
\hline 18) Nitrobenzene & 91.9 & 93.4 & 68.1 & 0.1 & & 0.1 \\
\hline 19) Isophorone & 96.1 & 95.7 & 65.1 & 0.1 & & 0.1 \\
\hline 20) 2--Nitrophenol & 96.9 & 100.4 & 0.2 & $4 \overline{3} .7$ & & 43.7 \\
\hline 21) 2,4-DImethylphenol & 92.4 & 93.8 & 34.2 & 17.7 & 0.3 & 18.0 \\
\hline 22) Benzole acld & 100.1 & 99.9 & & 19.5 & & 19.5 \\
\hline 23) bls(2-Chloroethoxy)methane & 95.9 & 99.4 & 69.2 & 0.2 & & 0.2 \\
\hline 24) 2,4-Dlchlorophenol & 93.6 & 102.5 & 0.5 & 46.9 & & 46.9 \\
\hline 25) 1,2,4-Trichlorobenzene & 86.9 & 92.3 & 64.5 & 0.1 & 50.4 & 50.6 \\
\hline 26) Naphthalene & 91.0 & 96.4 & 65.4 & 0.7 & 51.9 & 52.6 \\
\hline 27) 4 -Chloroaniline & 49.7 & 37.3 & 19.6 & 0.1 & & 0.1 \\
\hline 28) Hexachlorobutadiene & 87.6 & 94.4 & 62.0 & 0.1 & 42.4 & 42.5 \\
\hline 29) 4-Chiloro-3-methylphenol & 99.4 & 101.6 & 7.8 & 42.8 & & 42.8 \\
\hline 30) 2-Methylnaphthalene & 92.0 & 97.5 & 68.0 & 0.2 & 60.5 & 60.7 \\
\hline 31) Acenaphthene-d10 & 100.0 & 100.0 & 100.0 & 100.0 & 100.0 & 100.0 \\
\hline 32) Hexachlorocyclopentadlene & 93,3 & 96.3 & 50.4 & & 14.0 & 14.0 \\
\hline 33) 2,4,6-Trichlorophenol & 102.0 & 108.0 & & 48.6 & & 48.6 \\
\hline 34) 2,4,5-Trichlorophenol & 93.8 & 99.9 & & 45.9 & & 45.9 \\
\hline 35) 2-Chloronaphthalene & 90.6 & 96.3 & 68.5 & 0.2 & 62.9 & 63.0 \\
\hline 36) \#2-Fluoroblphenyl & 88.3 & 99.0 & 72.9 & 0.2 & 63.3 & 63.5 \\
\hline 37) 2-Nitroanilline & 106.7 & 104.1 & 80.2 & 0.1 & & 0.1 \\
\hline 38) Dlmethylphthalate & 98.9 & 105.5 & 73.6 & 0.2 & & 0.2 \\
\hline 39) Acenaphthylene & 93.1 & 101.3 & 69.1 & 0.1 & 64.3 & 64.4 \\
\hline 40) 2,6-Dinitrotoluene & 98.9 & 101.2 & 77.2 & & & \\
\hline 41) 3-Nitroanilln & 100.0 & 71.2 & 40.4 & & & \\
\hline
\end{tabular}


IABLE 5. (contd)

\begin{tabular}{|c|c|c|c|c|c|c|}
\hline $\begin{array}{l}\text { Sample: PNL Blank Spike Dupllcate } \\
\text { No. Compound Name }\end{array}$ & Step 1 & Re-.step 1 & P2BN & $P 2 A$ & P3BN & $\begin{array}{r}\text { Total } \\
\text { Recovery }\end{array}$ \\
\hline 42) Acenaphthene & 97.0 & 102.3 & 75.7 & 0.2 & 71.3 & 71.5 \\
\hline 43) 2,4-Dinitrophenol & 119.9 & 112.5 & & 22.8 & & 22.8 \\
\hline 44) 4-Nitropheriol & 97.9 & 87.2 & & 44.7 & & 44.7 \\
\hline 45) Dlbenzofuran & 97.0 & 101.1 & 74.8 & 0.2 & 71.0 & 71.1 \\
\hline 46) 2,4-Dinitrotoluene & 99.6 & 105.0 & 79.0 & 0.1 & & 0.1 \\
\hline 47) Dlethylphthalate & 104.9 & 106.7 & 81,6 & 0.2 & 0.3 & 0.5 \\
\hline 48) 4-Chlorophenyl-phenylether & 61.4 & 63.7 & 45.2 & & 47.1 & 47.1 \\
\hline 49) Fluorene & 95.3 & 103.2 & 77.0 & 0.2 & 73.6 & 73.7 \\
\hline 50) 4-Nitroaniline & 69.6 & 100.0 & 67,4 & & & \\
\hline 51) *Phenanthrene - d10 & 100,0 & 100.0 & 100.0 & 100.0 & 100.0 & 100.0 \\
\hline 52) $4,6-D \ln \mid$ tro-2-methylphenol & 112.4 & 110.3 & & 40.8 & & 40.8 \\
\hline 53) $N$-Nitrosodlphenylamine & 106.3 & 116.1 & 75.2 & 0.1 & 0.1 & 0.2 \\
\hline 54) \#2,4,6-Trlbromophenol & 104.6 & 118.1 & & 49.0 & & 49.0 \\
\hline 55) 4-Bromophenyl-phenylether & 93.4 & 99.6 & 67.9 & 0.1 & 76.5 & 76.6 \\
\hline 56) Hexachlorobenzeno & 95.0 & 103.6 & 73.0 & 0.1 & 68.9 & 69.0 \\
\hline 57) Pentachlorophenol & 105.6 & 116.0 & & 49.4 & & 49.4 \\
\hline 58) Phenanthrene & 98.9 & 101.6 & 75.6 & 0,2 & 80.7 & 80.9 \\
\hline 59) Anthracene & 96.1 & 100.3 & 72.5 & 0.2 & 76.0 & 76.2 \\
\hline 60) DI-n-butylphthalate & 99.7 & 101.8 & 76.3 & 0.2 & 0.0 & 0.3 \\
\hline 61) Fluoranthene & 96.8 & 98.9 & 73.2 & 0,2 & 85.9 & 86.1 \\
\hline 62) *Chrysene-d12 & 100.0 & 100.0 & 100.0 & 100.0 & 100.0 & 100.0 \\
\hline 63) Pyrene & 91.1 & 100.4 & 81.5 & 0.2 & 91.1 & 91.3 \\
\hline 64) \#Terphenyl-d14 & 79.4 & 90.8 & 76.9 & 0.3 & 83.6 & 83,9 \\
\hline 65) Butylbenzylphthalate & 92.6 & 99.7 & 86.4 & 0.2 & & 0.2 \\
\hline 66) 3,3-Dichlorobenzidine & 99.9 & 78.1 & 83.8 & & & \\
\hline 67) Benzo(a)anthracerie & 93.9 & 100.6 & 87.1 & 0.3 & 91.6 & 91.9 \\
\hline 68) Chryseng & 84.9 & 91.8 & 83.2 & 0.3 & 96.2 & 96.5 \\
\hline 69) Bls(2-Ethylhexyl)phthalate & 86.9 & 96.2 & 81.0 & 0.3 & 1.9 & 2.1 \\
\hline 70) *Perylene-d12 & 100.0 & 100.0 & 100.0 & 100,0 & 100.0 & 100.0 \\
\hline 71) DI-n-octylphthalate & 90.3 & 105.7 & 74.5 & 0.2 & 0.1 & 0.2 \\
\hline 72) Benzo(b)fluoranthene & 112.7 & 115.1 & 77.6 & 0.2 & 76.5 & 76.7 \\
\hline 73) Benzo (k)fluoranthene & 81.4 & 117.9 & 75.9 & 0.2 & 70.2 & 70.4 \\
\hline 74) Benzo(a)pyrene & 99.1 & 109.2 & 74.6 & 0.2 & 76.3 & 76.5 \\
\hline 75) Indeno(1,2,3-cd)pyrene & 118.1 & 125.0 & 81.4 & 0.1 & 81.0 & 81.1 \\
\hline 76) Dibenz $(a, h)$ anthracene & 93.4 & 104.9 & 72.1 & 0.1 & 75.6 & 75.7 \\
\hline 77) Benzo(g,h,i)perylene & 107,4 & 114.1 & 83.8 & 0.2 & 81.7 & 81.8 \\
\hline
\end{tabular}

\begin{tabular}{|l|l|l|l|}
\hline $\mathrm{NPH}$ (Paraffin Hydrocarbon, C12-C14) & & & \\
\hline
\end{tabular}

* Compound is an Internal Standard

\# Compound ls a Surrogate Splke 
TAB E 6. Percent Recovery Results for Method B1ank, EPA 3630

Cleanup Method

\begin{tabular}{|c|c|c|c|c|c|c|c|}
\hline $\begin{array}{l}\text { Sample: EPA Mothod Blank } \\
\text { No. Compound Name }\end{array}$ & Step 1 & Re-Stop 1 & E2N & E2A & $\mathrm{E2B}$ & E3N & $\begin{array}{r}\text { Total } \\
\text { Rooovery }\end{array}$ \\
\hline 1) *1,4-Dichlorobenzene-d4 & 100.0 & 100.0 & 100,0 & 100.0 & 100.0 & 100.0 & 100.0 \\
\hline 2) \#2-Fluorophanol & 99.9 & 101.4 & 0.1 & 44.0 & & & 44.0 \\
\hline 3) \#Phanol-d5 & 118.4 & 115.5 & 0.4 & 47.8 & & & 47,8 \\
\hline 4) Phenol & & & & & & & \\
\hline 5) blo(2-Chlornothyl)othor & & & & & & & \\
\hline 6) 2-Chlorophend & & & & & & & \\
\hline 7 1, 3-Dlohlorobenzeno & & & & & & & \\
\hline 8) 1,4-Dlohlorobenzena & & & & & & & \\
\hline 9) Benzylaloohol & & & & & & & \\
\hline 10) 1,i-Dlohlorobenzens & & & & & & & \\
\hline 11) 2-Methylphonol & & & & & & & \\
\hline 12 ble(2-chlorolsopropyllether & & & & & & & \\
\hline 13) 4-Mothylphenol & & & & & & & \\
\hline 14) $N$-Nitroso-dl-n-popylamine & & & & & & & \\
\hline 15) Hexachloroetharie & & & & & & & \\
\hline 16) Naphthalene-d8 & 100.0 & 100,0 & 100,0 & 100.0 & 100.0 & 100.0 & 100,0 \\
\hline 17) \#Nitrobenzene-d5 & 92.1 & 95.9 & 28.9 & 0.7 & 0.2 & 0.9 & 1.8 \\
\hline 18) Nitrobenzeno & & & & & & & \\
\hline 19) loophorone & & & & & & & \\
\hline 20) 2-Nitrophenol & & & & & & & \\
\hline 2.1) 2,4-Dimethylphenol & & & & & & & \\
\hline 22) Benzolo aold & & & & & & & \\
\hline 23) blo(2-Chloroethoxy)methane & & & & & & & \\
\hline 24) 2,4-Dlchlorophenol & & & & & & & \\
\hline 25) 1,2,4-Trichlorobenzene & & & & & & & \\
\hline 26) Naphthaleno & & & & & & & \\
\hline 27) 4-Chloroanlline & & & & & & & \\
\hline 28) Hexachlorobutadiene & & & & & & & \\
\hline 29) 4-Chloro-3-methylphenol & & & & & & & \\
\hline 30) 2-Methylnaphthalene & & & & & & & \\
\hline 31) "Acenaphthene-d10 & 100.0 & 100.0 & 100,0 & 100.0 & 100.0 & 100.0 & 100.0 \\
\hline 32) Hexachlorocyolopentadiens & & & & & & & \\
\hline 3.3) $2,4,6$-Trichlorophend & & & & & & & \\
\hline 34) 2,4,5-Triohlorophenol & & & & & & & \\
\hline 35 2-Chloronaphthalene & & & & & & & \\
\hline 35) \#2-Fluorobiphenyl & 92.3 & 102.7 & 37.7 & 2.2 & 2.1 & 34.7 & 39.0 \\
\hline 37) 2-Nitroanilline & & & & & & & \\
\hline 38) D/methylphthalate & & & & & & & \\
\hline 39) Acenaphthylene & & & & & & & \\
\hline 40) 2,6-Dinitrotoluene & & & & & & & \\
\hline 41) 3-Nitroanillino & & & & & & & \\
\hline
\end{tabular}


IABLE 6. (contd)

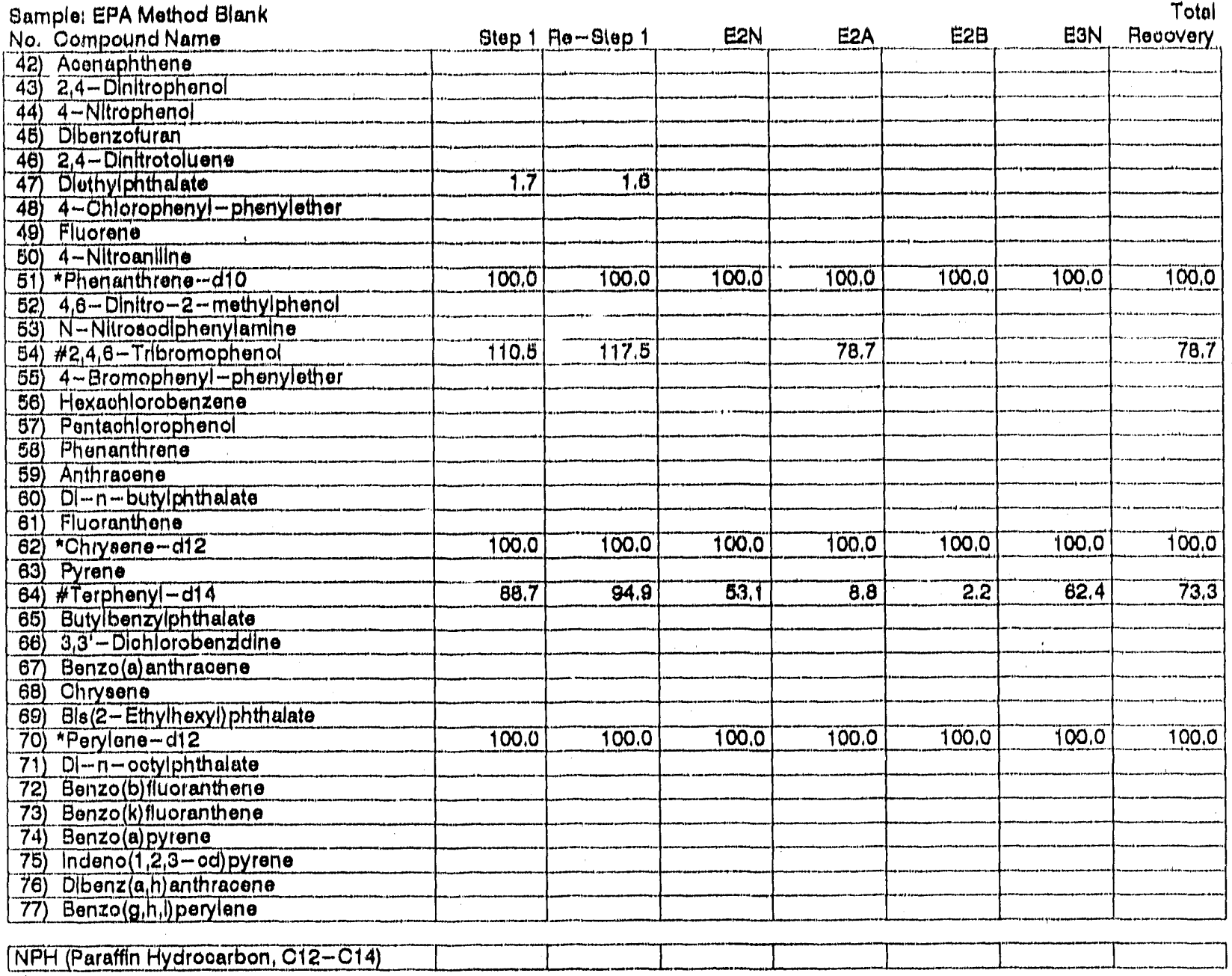

- Compound is an Intemal Btandard

* Compound lo a Surrogato Splko 


\section{IABLEE 7. Percent Recovery Results for Blank Sp 1k日, EPA 3630}

Cleanup Method

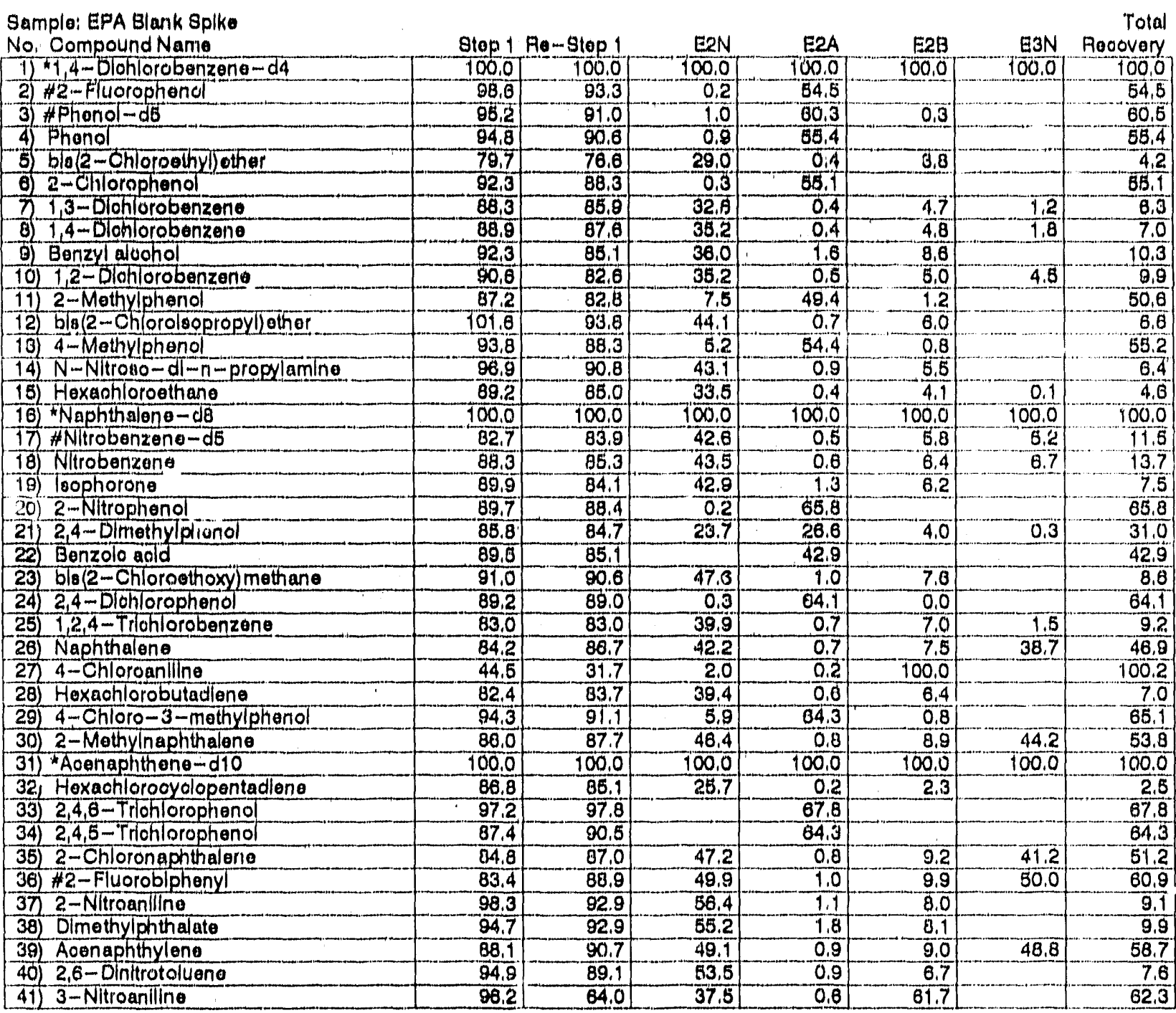


IABLE I. (contd)

\begin{tabular}{|c|c|c|c|c|c|c|c|}
\hline $\begin{array}{l}\text { Sample: EPA Blank Bpike } \\
\text { No. Compound Name }\end{array}$ & Stop 1 & Re-. Step 1 & E2N & E2A & $\underline{2} 28$ & E3N & $\begin{array}{l}\text { Total } \\
\text { Beoovory }\end{array}$ \\
\hline 42) Aoonaphthone & $92, \pi$ & 91.8 & 54,3 & 1.1 & 10,4 & 54.2 & 65.7 \\
\hline 43) 2,4-Dinttrophono & 106.8 & 93,7 & & 58,7 & & & 58.7 \\
\hline 44) 4-Nitrophenol & 100,2 & 97.5 & & 69,4 & & & 69,4 \\
\hline 45) Dlbenzofuran & 90,3 & 92.0 & 61,2 & 1,2 & 10,7 & 66.1 & 60,8 \\
\hline 46) 2,4 -Dinitrotoluene & $93, \theta$ & 94.0 & 84.0 & 1,6 & 6,8 & & 8,4 \\
\hline 47 Dlethylphthalalo & 99.0 & 96.8 & 59,6 & 2,2 & $8, \overline{6}$ & 0.2 & 10,8 \\
\hline 48) 4-Chlorophenyl-phenylether & $B 8,6$ & 57.4 & 33,2 & 0.8 & 7.9 & 36.8 & 44.8 \\
\hline 49) Fluorena & 91.1 & 91.1 & 00,3 & 1,4 & T1.2 & 67.4 & 70,0 \\
\hline 50) 4-Nitroanlline & 83.0 & 86.8 & 48,1 & 0.5 & 11.8 & & 12.3 \\
\hline 51) Phenanthreno-d10 & 100,0 & 100,0 & 100,0 & 100.0 & 100.0 & 100.0 & 100.0 \\
\hline (52) 4,6 -Dinttro-2-methylphanol & 100,3 & 96,2 & & 68,7 & & & 08.7 \\
\hline 53$) \mathrm{N}-$ Nitrosodiplienylemine & 101.4 & 107.0 & 67.7 & .0 .8 & 12.6 & 0,0 & 19.4 \\
\hline 54) \#2,4,6-tribromophenol & 98,6 & 105.2 & & 77.7 & & & 77.7 \\
\hline 55$)$ - Bromophieny-phenylother & 88.2 & 91.4 & 54,7 & 1,5 & 11.2 & 59.5 & 72.2 \\
\hline 56) Hoxachlorobenzeno & 92.8 & 93.8 & 58.0 & 1.7 & 9.3 & 1.4 & 12.4 \\
\hline 57) Pentachlorophonol & 96,0 & 102.4 & & 74,3 & & & 74.3 \\
\hline 58 Phonanthrento & 92.4 & 93.1 & 59,2 & 1,8 & 11,3 & 63.6 & 78.7 \\
\hline 59) Anthraoone & 91.1 & 92.1 & 50,2 & 1.7 & 10.7 & 56.8 & 68.2 \\
\hline 60) DI-n-butylphthalate & 92,3 & 93.0 & 61,8 & 2.6 & 9,6 & & 12.3 \\
\hline 61) Fluorantheno & 91,8 & 90.0 & 5,6 & 2.0 & 10.0 & 67,9 & 79.9 \\
\hline 82) "Chryseno-d12 & 100.0 & 100,0 & 100,0 & 100,0 & 100.0 & 100.0 & 100.0 \\
\hline 63) Pyrone & 94,9 & 88.7 & 56,8 & 2.1 & 9.2 & 66,3 & 77.6 \\
\hline 64) \#terphenyl-di4 & 81.2 & 81.9 & 53.8 & 2.4 & 9.8 & 01.6 & 73.6 \\
\hline 65) Butylbenzylphthalate & 95.9 & 88.6 & 59,8 & 2.7 & 8.9 & & 11.5 \\
\hline 66) 3,3-Dlohlorobenzdine & 100,0 & 71.4 & 29.1 & & 24,8 & & 24.8 \\
\hline 67) Benzo(a) anthraoone & 96.2 & 90.5 & 57.7 & 2.2 & 9.0 & 66.6 & 77.8 \\
\hline 68) Chryseng & 88,7 & 84,4 & E7.0 & 2.5 & 9.7 & 68,5 & 80.6 \\
\hline 69) Blo(2-Ethylhexyl)phthalate & 88.4 & 87.4 & 158.5 & 2.9 & 39.2 & 0.2 & 42.2 \\
\hline 70) Peryleno-di2 & 100.0 & 100.0 & 100,0 & 100.0 & 100.0 & 100.0 & 100.0 \\
\hline 71) $D 1-n$-octylphthalate & 84.0 & 93.7 & $68, \overline{5}$ & 2.6 & 11.8 & & 14.2 \\
\hline 72) Benzo(b)fluoranthene & 98.4 & 98,0 & 59.1 & 1.8 & 8,7 & 55.4 & 65.9 \\
\hline 73$)$ Berizo (k) fluoranthone & 70.7 & 89,9 & 56.5 & 2.5 & 9.7 & 55.3 & 67.4 \\
\hline 74) Benzo (a) pyreno & 91.0 & 96,4 & 58.9 & 2.0 & 8.8 & 53,6 & 64,4 \\
\hline 75) Induno $(1,2,3-c$ d) pyrene & 107.4 & 104.2 & 62,3 & 1.9 & 8.6 & $56, \bar{\theta}$ & B7.1 \\
\hline 76) Dlbenz $a, h)$ an thracene & 85,8 & 92.4 & 54.6 & 1.7 & 7.5 & 53.8 & 62.9 \\
\hline 77 Benzo $(g, h, 1)$ perylene & 99.7 & 98.1 & 60.9 & 1.9 & 8.3 & 51.7 & 61.8 \\
\hline
\end{tabular}

NPH (Paraffin Hydrooarbon, C12-C14)

$+$

"Compound la an Internal Standard

* Compound lie a Surroyato Spiko 
TABLE 8. Percent Recovery Results for Blank Splke Duplicate, EPA 3630

Cleanup Method

\begin{tabular}{|c|c|c|c|c|c|c|c|}
\hline $\begin{array}{l}\text { Samplo: EPA Blank Splke Duplloate } \\
\text { No. Compound Name }\end{array}$ & Stop 1 & He - Step 1 & E2N & E2A & E2B & E3N & $\begin{array}{r}\text { Total } \\
\text { Rouovery }\end{array}$ \\
\hline 1) 1,4 -Dlohlorobenzene-d4 & 100,0 & 100,0 & 100.0 & 100.0 & 100.0 & 100.0 & 100,0 \\
\hline (4) \#Fluorophenol & 107,3 & 104.9 & 0.2 & 50.8 & & & 60.9 \\
\hline 3) \#pheno-d5 & 101,4 & 102,0 & 1.3 & 54.6 & 0.1 & & 64.7 \\
\hline 4) Phenol & 101.6 & 103,6 & 1.3 & 52.1 & & & 52.1 \\
\hline (5) blo(2-chloroethyl)ether & 85,1 & 87.4 & 3,3 & 0.2 & & & 0.2 \\
\hline 6) 2-Chlorophenol & 98.7 & 98,4 & 0,3 & 61.6 & & & 61.6 \\
\hline n 1,3-Dlohlorobenzene & 100.7 & 97.2 & 42.6 & 0.2 & 1.7 & 7.6 & 3,4 \\
\hline 8) 1,4-Dlohlorobenzene & 91.6 & 96,4 & A6, 1 & 0.2 & 1,6 & 2.3 & 4,1 \\
\hline 9) Benzyl alcohol & 100,2 & 86.5 & 47,8 & 1,2 & 4,2 & & 6.3 \\
\hline 10) 1,2-Dlohlorobenzeno & 96.6 & 91.4 & 45.6 & 0.2 & 1.7 & 5.3 & 7.1 \\
\hline 11) 2-Mothylphenol & 93.1 & 94,8 & 0,3 & 45,4 & 0.4 & & 45,8 \\
\hline 12) bla(2-Chlorolsopropyl)ether & 108,5 & 103.9 & 58.4 & 0.3 & 2.1 & & 2.4 \\
\hline 13) 4-Mathylphenol & 103.2 & 100.1 & 6.4 & 49,0 & 0.3 & & 49.2 \\
\hline 14) $N-N \mid t r o s o-d 1-n-p r o p y l a m l n e$ & 102.8 & 99.7 & 50.7 & 0.5 & 1.8 & & 2.2 \\
\hline 15) Hexaohloroethane & $\$ 5.0$ & 96,1 & 45.0 & 0.1 & 1.5 & & 1,6 \\
\hline 16) Naphthalene-d8 & 100,0 & 100.0 & 100,0 & 100.0 & 100,0 & 100,0 & 100,0 \\
\hline 17) ANitrobenzone-d5 & 80.0 & 95,5 & 51.1 & 0.2 & 1.8 & 6.7 & 8,7 \\
\hline 18) Nltrobenzene & 01.9 & 93,4 & 52,3 & 0.2 & 1.9 & 8.7 & 10,0 \\
\hline 19) leophorone & 96.1 & 95.7 & 49,6 & 0.8 & 2.0 & & 2,8 \\
\hline 20) 2-Nitrophenol & 99.9 & 100.4 & 0.1 & 56.1 & & & 85.1 \\
\hline 24) 2,4-Dimethylphonol & 92.4 & 93.8 & 27,1 & 22.5 & 1.2 & & 23.7 \\
\hline 22) Benzolo nold & 100,1 & 99.9 & & 42.5 & & & 42,5 \\
\hline 23) bis $(2-$ Chloroethoxy) mathane & 95.9 & 98,4 & 35.1 & 0.5 & 2.3 & & 2.8 \\
\hline 24) 2,4 -Dlohlorophenol & 93.6 & 102.5 & 0.3 & 55.7 & & & 55.7 \\
\hline 25) 1,2,4-Thohlorobenzeno & 86.9 & 92.3 & 47.0 & 0.3 & 2.0 & 2.0 & 4.3 \\
\hline 26) Naphttialene & 91.0 & 98.4 & 49,9 & 0,8 & 2.2 & 39.5 & 42.6 \\
\hline 27) 4-Chloroanillno & 49,7 & $3 \%, 3$ & 1.8 & 0.4 & 96.0 & & 95.4 \\
\hline 28) Hexachlorobutadiene & 87.6 & 94,4 & 47.7 & 0.3 & 2.0 & & 2.3 \\
\hline 29) 4-Chloro-3-methylphenol & 99,4 & 101.6 & 6.3 & 56.7 & 0.2 & & 55.9 \\
\hline 30) 2-Mothylnaphthalena & 92.0 & 97.5 & 51.6 & & 2.1 & 42.9 & 45.0 \\
\hline 31) Acenaphthene-d10 & 100.0 & 100.0 & 100.0 & 100.0 & 100.0 & 100.0 & 100.0 \\
\hline 32) Hexachlorooyclopentadiene & 93,3 & 98.3 & 32.7 & & 0.4 & & 0.4 \\
\hline 33) 2,4,6-Triohlorophenol & 102.0 & 108.0 & & 58.7 & & & 58.7 \\
\hline 34) 2,4,5-Triohlorophenol & 93.8 & 99.9 & & 65.7 & & & 55.7 \\
\hline 35) 2-Chloronaphthalone & 90.6 & 96,3 & 50.3 & 0.5 & 2,1 & 32.7 & 35.3 \\
\hline 36) "2-fluorobiphanyl & 88.3 & 99,0 & 50.4 & 0.5 & 2.1 & 44.1 & 46.7 \\
\hline 37) 2 -Nitroanilirio & 108.7 & 104.1 & 69,3 & 0.7 & 1.5 & & 2.2 \\
\hline 38) Dimothylphthalate & 98,9 & 105,5 & 54,9 & 1.3 & 2.2 & & 3,4 \\
\hline 39) Aoonaphthylene & 93.1 & 101.3 & 51.5 & 0,6 & 2.0 & 44.7 & 47,3 \\
\hline 40) 2,6-Dinitrotoluene & 98.9 & 101.2 & 54,3 & 0.6 & 1.2 & & 9.8 \\
\hline 41) 3-Nitroaniline & 100.0 & 71.2 & 46.6 & 0.4 & 47.6 & & 49.0 \\
\hline
\end{tabular}


TABLE 8. (contd)

\begin{tabular}{|c|c|c|c|c|c|c|c|}
\hline $\begin{array}{l}\text { Sample: EPA Blank Spike Duplicate } \\
\text { No. Compound Name }\end{array}$ & Step 1 & Re-Step 1 & E2N & E2A & E2B & ESN & $\begin{array}{r}\text { Total } \\
\text { Recovery }\end{array}$ \\
\hline 42) Acenaphthene & 97.0 & 102.3 & 58.0 & 0.7 & 2.4 & 49.6 & 52.8 \\
\hline 43) 2,4-Dinitrophenol & 119.9 & 112.5 & & 55.0 & & & 55.0 \\
\hline 44) 4-Nitrophenol & 97.9 & 87.2 & & 65.6 & & & 65.6 \\
\hline 45) Dibenzofuran & 97.0 & 101.1 & 53.2 & 0.8 & 2.3 & 50.5 & 53.6 \\
\hline 46) 2,4-Dinitrotoluene & 99.6 & 105.0 & 54.8 & 1.2 & 1.2 & & 2.3 \\
\hline 47 Diethylphthalate & 104.9 & 106.7 & 59.5 & 1.5 & 2.5 & 0.2 & 4.2 \\
\hline 48) 4-Chlorophenyl-phenylether & 61.4 & 63.7 & 34.5 & 0.6 & 1.5 & 33.9 & 36.0 \\
\hline 49) Fluoreno & 95.3 & 103.2 & 57.9 & 1.0 & 2.2 & 51.4 & 54.6 \\
\hline 50) 4-Nitroaniline & 69.6 & 100.0 & 61.5 & 0.4 & 3.6 & & 3.9 \\
\hline 51) Phenanthrene-d10 & 100.0 & 100.0 & 100.0 & 100.0 & 100.0 & 100.0 & 100.0 \\
\hline 52.) 4,6-Dinttro-2-methylphenol & 112.4 & 110.3 & & 66.5 & & & 66.5 \\
\hline 53) N-Nitrosodiphenylamine & 106.3 & 116.1 & 66.7 & 0.6 & 2.9 & 7.3 & 10.7 \\
\hline 54) \#2,4,6-Tribromophenol & 104.6 & 118.1 & & 68.9 & & & 68.9 \\
\hline 55) 4-Bromophenyl-phenylether & 93.4 & 99.6 & 55.5 & 1.2 & 2.3 & 56.1 & 59.5 \\
\hline 56) Hexachlorobenzene & 95.0 & 103.6 & 57.0 & 1.2 & 2.5 & 0.5 & 4.1 \\
\hline 57$)$ Pentachlorophenol & 105.6 & 116.0 & & 67.2 & & & 67.2 \\
\hline 58) Phenanthrene & 98.9 & 101.6 & 58.5 & 1.4 & 2.5 & 60.6 & 64.5 \\
\hline 59) Anthraceno & 96.1 & 100.3 & 54.8 & 1.3 & 2.3 & 57.0 & 60.6 \\
\hline 60) Di-n-butylphthalate & 99.7 & 101.8 & 62.8 & 1.8 & 3.0 & 0.0 & 4.9 \\
\hline 61) Fluoranthene & 96.8 & 98.9 & 57.4 & 1.5 & 2.5 & 64.9 & 68.8 \\
\hline 62) Chrysene-d12 & 100.0 & 100.0 & 100.0 & 100.0 & 100.0 & 100.0 & 100.0 \\
\hline 63) Pyrene & 91.1 & 100.1 & 54.5 & 1.5 & 2.3 & 67.3 & 71.1 \\
\hline 64) \#Terphenyl-di4 & 79.4 & 90.8 & 51.3 & 1.7 & 2.4 & 63.4 & 67.5 \\
\hline 65) Butylbenzylphthalate & 92.6 & 99.7 & 60.1 & 2.0 & 2.7 & & 4.8 \\
\hline 66) 3,3'-Dichlorobenzidine & 99.9 & 78.1 & 23.0 & & 5.2 & & 5.2 \\
\hline 67) Benzo (a)anthracene & 93.9 & 100.6 & 55.0 & 1.7 & 2.4 & 66.4 & 70.4 \\
\hline 68) Chrysene & 84.9 & 91.8 & 55.4 & 1.9 & 2.6 & 72.9 & 77.4 \\
\hline 69) Bis(2-Ethyihexyl)phthalate & 86.9 & 96.2 & 54.6 & 2.0 & 2.7 & 0.2 & 4.9 \\
\hline 70) *Perylene-d12 & 100.0 & 100.0 & 100.0 & 100.0 & 100.0 & 100.0 & 100.0 \\
\hline 71) DI-n-octylphthalate & 90.3 & 105.7 & 59.3 & 2.0 & 2.5 & & 4.5 \\
\hline 72) Benzo(b)fluoranthene & 112.7 & 115.1 & 55.6 & 1.4 & 2.2 & 54.4 & 58.0 \\
\hline 73) Benzo(k)fluoranthene & 81.4 & 117.9 & 62.4 & 1.9 & 2.7 & 55.3 & 59.9 \\
\hline 74) Benzo(a)pyrene & 99.1 & 109.2 & 56.2 & 1.5 & 2.3 & 51.7 & 55.5 \\
\hline 75) Indeno $(1,2,3$-cd)pyreno & 118.1 & 125.0 & 60.4 & 1.3 & 2.1 & 57.4 & 60.8 \\
\hline 76) Dibenz(a,h) anthracene & 93.4 & 104.9 & 54.2 & 1.2 & 1.8 & 52.4 & 55.5 \\
\hline I7) Benzo $(g, h, i)$ perylene & 107.4 & 114.1 & 59.2 & 1.3 & 2.2 & 55.1 & 58.5 \\
\hline
\end{tabular}

\begin{tabular}{|l|l|l|l|l|}
\hline NPH (Paraffin Hydrocarbon, C12-C14) I & \\
\hline
\end{tabular}

* Compound is an Internal Standard

* Compound la a Surrogate Spike 
TABLE 9. Percent Recovery Results for Blank Spike, Initial PNL Cleanup

Method, Followed by Increased Methylene Chloride Elution

Sample: PNL Blank Spike NPH (control)

No. Compound Name

Step P2BN

1) $* 1,4$-Dichlorobenzene-d4

2) \#2-Fluorophenol

3) \#Phenol-d5

4) Phenol

5) bis(2-Chloroethyl)ether

6) 2-Chlorophenol

7) 1,3-Dichlorobenzene

8) 1,4-Dichlorobenzene

9) Benzyl alcohol

10) 1,2-Dichlorobenzene

11) 2-Methylphenol

12) bis(2-Chloroisopropyl)ether

13) 4-Methylphenol

14) N-Nitroso-di-n-propylamine

15) Hexachloroethane

16) *Naphthalene-c8

17) \#Nitrobenzene-d5

18) Nitrobenzene

19) Isophorone

20) 2-Nitrophenol

21) 2,4-Dimethylphenol

22) Benzoic acid

23) bis(2-Chloroethoxy)methane

24) 2,4-Dichlorophenol

25) 1,2,4-Trichlorobenzene

26) Naphthalene

27) 4-Chloroaniline

28) Hexachlorobutadiene

29) 4-Chloro-3-methylphenol

30) 2-Methylnaphthalene

31) *Acenaphthene--d10

32) Hexachlorocyclopentadiene

33) 2,4,6-Trichloroplienol

34) 2,4,5-Trichlorophenol

35) 2-Chloronaphthalene

36) \#2-Fluorobiphenyl

37) 2-Nitroaniline

38) Dimethylphthalate

39) Acenaphthylene

40) 2,6-Dinitrotoluene

41) 3-Nitroaniline

\begin{tabular}{|c|c|c|c|c|}
\hline Step P2BN & $P 2 A$ & P3BN & P3BN2 & Recovery \\
\hline 100.0 & 100.0 & 100.0 & 100.0 & 100.0 \\
\hline 0.2 & 55.9 & 0.0 & 0.0 & 55.9 \\
\hline 4.2 & 58.7 & & & 58.7 \\
\hline & 54.3 & & 0.1 & 54.4 \\
\hline 71.9 & 0.5 & & 37.7 & 38.2 \\
\hline 0.9 & 57.2 & & & 57.2 \\
\hline 61.8 & 0.3 & 34.0 & & 34.4 \\
\hline 62.3 & 0.4 & 37.8 & & 38.1 \\
\hline 72.2 & 1.7 & & & 1.7 \\
\hline 60.9 & 0.4 & 37.6 & & 38.0 \\
\hline 25.0 & 43.3 & & 12.0 & 55.3 \\
\hline 84.3 & 0.6 & & 57.9 & 58.5 \\
\hline 19.9 & 48.4 & & 0.3 & 48.6 \\
\hline 79.6 & 0.9 & & & 0.9 \\
\hline 59.7 & 0.3 & 15.4 & & 15.7 \\
\hline 100.0 & 100.0 & 100.0 & 100.0 & 100.0 \\
\hline 71.5 & 0.5 & & 57.4 & 57.8 \\
\hline 73.6 & 0.5 & & 59.9 & 60.4 \\
\hline 69.3 & 1.5 & & & 1.5 \\
\hline 0.4 & 67.9 & & & 67.9 \\
\hline 53.4 & 15.3 & 0.3 & 23.1 & 38.7 \\
\hline & 52.7 & & & 52.7 \\
\hline 75.1 & 1.1 & & 65.5 & 66.5 \\
\hline 0.9 & 70.9 & & & 70.9 \\
\hline 68.6 & 0.7 & 48.5 & 0.0 & 49.2 \\
\hline 69.5 & 1.0 & 53.3 & 1.0 & 55.3 \\
\hline 102.5 & 0.6 & & 1.2 & 1.8 \\
\hline 68.8 & 0.7 & 19.9 & & 20.6 \\
\hline 18.1 & 59.4 & & 2.5 & 61.9 \\
\hline 71.0 & 1.0 & 59.1 & 1.4 & 61.5 \\
\hline 100.0 & 100.0 & 100.0 & 100.0 & 100.0 \\
\hline 61.5 & 0.3 & 14.5 & & 14.8 \\
\hline & 71.7 & & & 74.7 \\
\hline & 68.7 & & & 68.7 \\
\hline 72.0 & 1.3 & 61.7 & 0.1 & 63.0 \\
\hline 74.4 & 1.4 & 59.9 & 3.4 & 64.6 \\
\hline 84.2 & 1.6 & & 65.9 & 67.5 \\
\hline 78.9 & 2.8 & & & 2.8 \\
\hline 74.3 & 1.5 & 60.4 & 2.6 & 64.4 \\
\hline 77.2 & & & 70.6 & 70.6 \\
\hline 84.5 & 0.4 & 0.1 & 3.2 & 3.8 \\
\hline
\end{tabular}


IABLE 9. (contd)

Sample: PNL Blank Spike NPH (control)

No. Compound Name

42) Acenaphthene

43) 2,4-Dinitrophenol

44) 4-Nitrophenol

45) Dibenzofuran

46) 2,4-Dinitrotoluene

47) Diethylphthialate

48) 4-Chlorophenyl-phenylether

49) Fluorene

50) 4-Nitroaniline

51) *Phenanthrene-d10

52) 4,6-Dinitro-2-methylphenol

53) $\mathrm{N}$-Nitrosodiphenylamine

54) \#2,4,6-Tribromophenol

55) 4-Bromophenyl-phenylether

56) Hexachlorobenzene

57) Pentachlorophenol

58) Phenanthrene

59) Anthracene

60) Di-n-butylphthalate

61) Fluoranthene

62) *Chrysene-d12

63) Jyrene

64) \#Terphenyi-d14

65) Butylbenzylphthalate

66) $3,3^{\prime}$-Dichlorobenzidine

67) Benzo(a)anthracene

68) Chrysene

69) Bis(2-Ethylhexyi) phthalate

70) *Perylene-d12

71) Di-n-octylphthalate

72) Benzo(b)fluoranthene

73) Benzo(k)fluoranthene

74) Benzo (a)pyrene

75) Indeno (1,2,3-cd)pyrene

76) Dibenz(a,h)anthracene

77) Benzo(g,h,i)perylene

Step P2BN P2A P3BN P3BN2 Recovery

Step P2BN P2A P3BN P3BN2 Recovery

Step P2BN P2A P3BN P3BN2 Recovery

Total

NPH (Paraffin Hydrocarbon, C12-C14)

\begin{tabular}{r|r}
81.5 & 1 \\
\hline & 78.9 \\
\hline
\end{tabular}

78.9

5.8

77.6

2.

81.6

48.6

78.4

100.0

100.0

3.4

3.2

2.3

0.9

100.0

85.1

91.2

\begin{tabular}{l}
0.8 \\
\hline
\end{tabular}

0.8

77.7

9.6

2

2.7

2.7

2.7
95.2

76.2

6.2

2.8

2.6

2.6

76.7

\begin{tabular}{r|r}
\hline & 76 \\
\hline & 100 \\
\hline
\end{tabular}

00.0

77.4

2.9

2.9

2.9

3.0

\begin{tabular}{l|l}
\hline & 77 \\
\hline & 75 \\
\hline
\end{tabular}

77.5

5.3

7.1

.

late

78.9

78.9

$\begin{array}{r}100.0 \\ \hline 67.9\end{array}$

67.9

\begin{tabular}{l}
80.8 \\
70.5 \\
\hline 75.4
\end{tabular}

75.4

5.4

$\frac{71.3}{80.0}$

$\begin{array}{r}\hline 3.3 \\ \hline 3.1 \\ 3.4 \\ \hline 4.0 \\ \hline 100.0 \\ \hline 3.5 \\ \hline 3.0 \\ \hline 2.8 \\ \hline 2.7 \\ \hline 2.8 \\ \hline 2.5 \\ \hline 2.8\end{array}$

\begin{tabular}{l|l}
3.3 & 65.0 \\
\hline 3.3 & 0.0
\end{tabular}

\begin{tabular}{l|l}
3.1 & 69.4
\end{tabular}

$3.4 \quad 73.5$

73.5

\begin{tabular}{r|r}
4.0 & 0.2 \\
\hline 000 & 100.0
\end{tabular}

0.2

\begin{tabular}{r|r}
3.5 & 0.0 \\
\hline 3.0 & 63.1
\end{tabular}

\begin{tabular}{l|l}
3.0 & 63.1 \\
\hline 28 & 68.0
\end{tabular}

3.1

\begin{tabular}{l|l}
2.8 & 68.0 \\
\hline 2.7 & 65.0
\end{tabular}

$2.8 \quad 64.4$

2.5

64.4

63.4

0.1

\begin{tabular}{l|l|}
\hline 3.1 & 71.6 \\
\hline & 78.9 \\
\hline
\end{tabular}

78.9

75.8

\begin{tabular}{|c|c|c|}
\hline & & 75.8 \\
\hline 7 & 6.9 & 69 \\
\hline & 67.1 & 69. \\
\hline
\end{tabular}

* Compound is an Internal Standard

\# Compound is a Surrogate Spike 
TABLE 10. Percent Recovery Results for Blank Spike with NPH, Initial PNL Cleanup Method, Followed by Increased Methylene Chloride Elution

Sample: PNL Blank Spike + NPH No. Compound Name

\begin{tabular}{|c|c|c|c|c|c|}
\hline & & & & & \\
\hline 1) *1,4-Dichlorobenzene-d4 & 100.0 & 100.0 & 100.0 & 100.0 & 100.0 \\
\hline 2) \#2-Fluoropheriol & 0.4 & 64.7 & 0.0 & 0.0 & 64.7 \\
\hline 3) \#Phenol-d5 & 3.6 & 65.8 & & & 65.8 \\
\hline 4) Phenol & 2.6 & 59.8 & & & 59.8 \\
\hline 5) bis(2-Chlornethyl)ether & 79.3 & 2.2 & & 46.2 & 48.4 \\
\hline 6) 2-Chlorophenol & 1.0 & 66.6 & & & 66.6 \\
\hline 7) 1,3-Dichlorobenzene & 69.1 & 1.4 & 46.7 & & 48.1 \\
\hline 8) 1,4-Dichlorobenzene & 73.9 & 1.6 & 48.0 & & 49.6 \\
\hline 9) Benzyl alcohol & 80.4 & 5.2 & & & 5.2 \\
\hline 10) 1,2-Dichlorobenzene & 69.9 & 1.6 & 48.3 & & 49.9 \\
\hline 11) 2-Methylphenol & 20.7 & 54.0 & & 7.9 & 62.0 \\
\hline 12) bis(2-Chloroisopropyl)ether & 96.6 & 2.6 & & 70.1 & 72.7 \\
\hline 13) 4-Methylphenol & 16.0 & 57.3 & & & 57.3 \\
\hline 14) $\mathrm{N}$-Nitroso-di-n-propylamine & 91.1 & 3.6 & & & 3.6 \\
\hline 15) Hexachloroethane & 69.1 & 1.1 & 46.2 & & 47.3 \\
\hline 16) *Naphthalene-d8 & 100.0 & 100.0 & 100.0 & 100.0 & 100.0 \\
\hline 17) \#Nitrobenzene-d5 & 82.4 & 2.4 & & 66.9 & 69.3 \\
\hline 18) Nitrobenzene & 80.4 & 2.8 & & 72.6 & 75.4 \\
\hline 19) Isophorone & 77.6 & 5.3 & & & 5.3 \\
\hline 20) 2-Nitrophenol & 0.4 & 78.9 & & & 78.9 \\
\hline 21) 2,4-Dimethylphenol & 54.0 & 28.0 & 0.3 & 18.8 & 47.0 \\
\hline 22) Benzoic acid & & 61.7 & & & 61.7 \\
\hline 23) bis(2-Chloroethoxy)methane & 81.9 & 4.6 & & 76.1 & 80.8 \\
\hline 24) 2,4-Dichlorophenol & 0.9 & 74.7 & & & 74.7 \\
\hline 25) 1,2,4-Trichlorobenzene & 75.0 & 2.9 & 64.6 & 0.1 & 67.6 \\
\hline 26) Naphthalene & 75.7 & 3.4 & 62.2 & 5.0 & 70.6 \\
\hline 27) 4-Chloroaniline & 24.3 & 0.6 & & 1.9 & 2.5 \\
\hline 28) Hexachlorobutadiene & 73.7 & 2.5 & 57.7 & & 60.3 \\
\hline 29) 4-Chloro-3-methylphenol & 14.9 & 69.6 & & 1.4 & 71.1 \\
\hline 30) 2-Methylnaphthalene & 55.5 & 4.7 & 63.5 & 5.9 & 74.1 \\
\hline 31) *Acenaphthene-d10 & 100.0 & 100.0 & 100.0 & 100.0 & 100.0 \\
\hline 32) Hexachlorocyclopentadiene & 62.2 & 1.3 & 44.8 & & 46.2 \\
\hline 33) 2,4,6-Trichlorophenol & & 83.9 & & & 83.9 \\
\hline 34) 2,4,5-Trichlorophenol & & 81.6 & & & 81.6 \\
\hline 35) 2-Chiloronaphthalene & 79.9 & 5.5 & 72.8 & 0.1 & 78.5 \\
\hline 36) \#2-Fluorobiphenyl & 82.7 & 6.1 & 69.1 & 9.5 & 84.7 \\
\hline 37) 2-Nitroaniline & 90.8 & 6.4 & & 76.3 & 82.7 \\
\hline 38) Dimethylphthalate & 81.2 & 8.2 & & & 8.2 \\
\hline 39) Acenaphthylene & 79.0 & 6.5 & 67.5 & 6.2 & 80.3 \\
\hline 40) 2,6-Dinitrotoluene & 82.1 & 6.1 & & 81.3 & 87.3 \\
\hline 41) 3 -Nitroaniline & 113.3 & 2.9 & & 12.9 & 15.8 \\
\hline
\end{tabular}


TABLE 10. (contd)

Sample: PNL Blank Spike + NPH

No. Compound Name

42) Acenaphthene

43) 2,4-Dinitrophenol

44) 4-Nitrophenol

45) Dibenzofuran

46) 2,4-Dinitrotoluene

47) Dlethylphthalate

48) 4-Chlorophenyl-phenylether

49) Fluorene

50) 4-Nitroanilline

51) *Phenanthrene-d10

52) 4,6-Dinitro-2-methylphenol

53) $\mathrm{N}$-Nitrosodiphenylamine

54) \#2,4,6-Tribromophenol

55) 4-Bromophenyl-phenylether

56) Hexachlorobenzene

57) Pentachlorophenol

58) Phenanthrene

59) Anthracene

60) Di-n-butylphthalate

61) Fluoranthene

62) *Chrysene-d12

63) Pyrene

64) \#Terphenyl-d14

65) Butylbenzylphthalate

66) 3,3'-Dichlorobenzidine

67) Benzo(a)anthracene

68) Chrysene

69) Bis(2-Ethylhexyl) phthalate

70) *Perylene-d12

71) Di-n-octylphthalate

72) Benzo(b)fluoranthene

73) Benzo(k)fluoranthene

74) Benzo(a)pyrene

75) Indeno (1,2,3-co)pyrene

76) Dibenz(a,h)anthracene

77) Benzo(g,h,l)perylene

\begin{tabular}{l|l}
76.5 \\
\hline
\end{tabular}

\begin{tabular}{l|r}
\hline & \\
\hline & 84.7
\end{tabular}

Step P2BN

P2A

Total

\begin{tabular}{|r|r|}
\hline 88.0 & 7.0 \\
\hline & 83. \\
\hline
\end{tabular}

7.0

80.9

85.1

94.8

0.9

154.7

100.0

$\quad 100.0$

2.8

$\begin{array}{r}86.8 \\ \hline\end{array}$

6.5

8.8

7.0
7.4

4.7

9

\begin{tabular}{l|l}
- & \\
\hline &
\end{tabular}

82.7

82.7

8.0

\begin{tabular}{l|l}
- & 100 \\
\hline
\end{tabular}

82.8

86.4

-

100

\begin{tabular}{l}
7.8 \\
\hline \\
\hline
\end{tabular}

P3BN P3BN2 Recovery

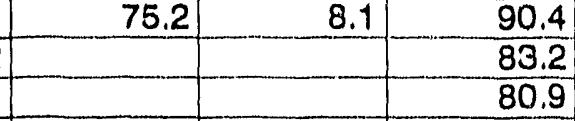

\begin{tabular}{r|r|r|r|}
\hline 7.2 & 70.5 & 12.2 & 89.9 \\
\hline 7.5 & & 75.3 & 82. \\
\hline
\end{tabular}

NPH (Paraffin Hydrocarbon, C12-C14)

86.8

85.6

100.0

79.2

9.3

6.3

6.3

8.9

79.2

8.8

$\frac{0.0}{0.8}$

$0.0 \quad 0.0$

0.8

0.1

0.8

0.8

7.6

3.0

85.2

$-100$

10.1
59.3

0.0

\begin{tabular}{l|l}
\hline 4 & \\
\hline 0 & \\
\hline 4 & \\
\hline
\end{tabular}

75.4

\begin{tabular}{l|r}
\hline & 100.0 \\
\hline
\end{tabular}

$\frac{8.0}{7.4}$

\begin{tabular}{r|r}
7.9 \\
8.0
\end{tabular}

\begin{tabular}{l|l}
\hline 0 & \\
\hline 4 & \\
\hline 6 &
\end{tabular}

89.2

5.4

63.8

77.2

11.3

7.9

78

\begin{tabular}{r|r}
77.2 & 10.3 \\
\hline 76.2 & 8.6 \\
\hline 0.0 & 0.0 \\
\hline 74.5 & 10.5 \\
\hline 100.0 & 100.0 \\
\hline 71.6
\end{tabular}

\begin{tabular}{r|r}
77.2 & 10.3 \\
\hline 76.2 & 8.6 \\
\hline 0.0 & 0.0 \\
\hline 74.5 & 10.5 \\
\hline 00.0 & 100.0 \\
\hline 71.6 & 10.8
\end{tabular}

100.0

100.0

\begin{tabular}{l}
$8.6-\frac{95.6}{92.1}$ \\
\hline-0.7
\end{tabular}

\begin{tabular}{|c|c|c|}
\hline 0 & 0.0 & 8.7 \\
\hline & 10.5 & 92.9 \\
\hline & 100.0 & 100.0 \\
\hline
\end{tabular}

* Compound is an Internal Standard

\# Compound is a Surrogate Spike 
TABLE 11. Percent Recovery Results for Blank Spike with NPH Duplicate, Initial PNL. Cleanup Method, Followed by Increased Methylene Chloride Elution

Sample: PNL Blank Spike + NPH Duplicate No. Compound Name

1) *1,4-Dichlorobenzene-d4

2) \#2-Fluorophenol

3) \#Phenol-d5

4) Phenol

5) bis(2-Chloroethyl)ether

6) 2-Chlorophienol

7) 1,3-Dichlorobenzene

8) 1,4-Dichlorobenzene

9) Benzyl alcohol

10) 1,2-Dichlorobenzene

11) 2-Methylphenol

12) bis(2-Chloroisopropyl)ether

13) 4-Methylphenol

14) $N$-Nitroso-di $-n$-propylamine

15) Hexachloroethane

16) *Naphthalene-d8

17) \#Nitrobenzene-d5

18) Nitrobenzene

19) Isophorone

20) 2-Nitrophenol

21) 2,4-Dimethylphenol

22) Benzolc acid

23) bis(2-Chloroethoxy)methane

24) 2,4-Dichlorophenol

25) 1,2,4-Trichlorobenzene

26) Naphthalene

27) 4-Chloroanilline

28) Hexachlorobutadiene

29) 4-Chloro-3-methylphenol

30) 2-Methylnaphthalene

31) *Acenaphthene-d10

32) Hexachlorocyclopentadiene

33) 2,4,6-Trichlorophenol

34) 2,4,5-Trichlorophenol

35) 2-Chloronaphthalene

36) \#2-Fluorobiphenyl

37) 2-Nitroaniline

38) Dimethylphthalate

39) Acenaphthylene

40) 2,6-Dinitrotoluene

41) 3-Nitroaniline

\begin{tabular}{|r|r} 
alene & 81.0 \\
\hline nyl & 83.7 \\
\hline & 93.6 \\
\hline & 86.3 \\
\hline & 80.0 \\
\hline & 87.3 \\
\hline
\end{tabular}

\begin{tabular}{|c|c|c|c|c|}
\hline P2BN & P2A & P3BN & P3BN2 & $\begin{array}{l}\text { Total } \\
\text { Recovery }\end{array}$ \\
\hline 100.0 & 100.0 & 100.0 & 100.0 & 100.0 \\
\hline 0.6 & 64.5 & 0.0 & 0.0 & 61.6 \\
\hline 4.1 & 66.5 & & & 66.5 \\
\hline 0.0 & 59.0 & & & 59.0 \\
\hline 80.4 & 0.8 & & 51.7 & 52.5 \\
\hline 1.2 & 64.3 & & & 64.3 \\
\hline 67.3 & 0.6 & 42.5 & 0.3 & 43.3 \\
\hline 76.1 & 0.6 & 44.4 & 0.3 & 45.2 \\
\hline 79.0 & 2.3 & & & 2.3 \\
\hline 69.0 & 0.6 & 43.3 & 0.3 & 44.2 \\
\hline 24.7 & 54.0 & & & 54.0 \\
\hline 90.8 & 0.9 & & 73.4 & 74.3 \\
\hline 17.2 & 57.1 & & & 57.1 \\
\hline 86.1 & 1.1 & & & 1.1 \\
\hline 68.6 & 0.4 & 37.7 & 0.3 & 38.4 \\
\hline 100.0 & 100.0 & 100.0 & 100.0 & 100.0 \\
\hline 83.3 & 0.7 & & 74.7 & 75.3 \\
\hline 79.8 & 0.7 & & 73.7 & 74.4 \\
\hline 76.5 & 1.6 & & & 1.6 \\
\hline 0.8 & 77.6 & & & 77.6 \\
\hline 56.1 & 24.5 & 0.4 & 1.0 & 25.9 \\
\hline 0.0 & 65.5 & & & 65.5 \\
\hline 84.0 & 1.3 & & 77.6 & 78.9 \\
\hline 1.3 & 75.2 & & & 75.2 \\
\hline 76.5 & 1.0 & 60.9 & 0.4 & 62.2 \\
\hline 77.2 & 1.0 & 60.4 & 0.5 & 61.9 \\
\hline 100.0 & 0.1 & & 2.4 & 2.5 \\
\hline 76.9 & 0.8 & 51.0 & 0.4 & 52.2 \\
\hline 15.9 & 69.0 & & & 69.0 \\
\hline 63.4 & 1.2 & 64.4 & 0.4 & 66.1 \\
\hline 100.0 & 100.0 & 100.0 & 100.0 & 100.0 \\
\hline 76.1 & 0.3 & 37.2 & 0.0 & 37.5 \\
\hline 0.0 & 83.9 & & & 83.9 \\
\hline 0.0 & 81.6 & & & 81.6 \\
\hline 81.0 & 1.3 & 71.4 & 0.4 & 73.1 \\
\hline 83.7 & 1.3 & 73.5 & 0.5 & 75.4 \\
\hline 93.6 & 1.4 & & 86.1 & 87.5 \\
\hline 86.3 & 2.5 & & & 2.5 \\
\hline 80.0 & 1.4 & 73.9 & 0.6 & 75.9 \\
\hline 87.3 & 1.3 & & 90.0 & 91.3 \\
\hline 100.0 & $\overline{0.4}$ & & & 0.4 \\
\hline
\end{tabular}


TABLE 11. (contd)

Sample: PNL Blank Spike + NPH Duplicate No. Compound Narne

42) Acenaphthene

43) 2,4-Dinitrophenol

44) 4-Nitrophenol

45) Dibenzofuran

46) 2,4-Dinitrotoluene

47) Diethylphthalate

48) 4-Chlorophenyl-phenylether

49) Fluorene

50) 4-Nitroaniline

51) *Phenanthrene-d10

52) 4,6-Dinitro-2-methylphenol

53) $\mathrm{N}$-Nitrosodiphenylamine

54) \#2,4,6-Tribromophenol

55) 4-Bromophenyi-phenylether

56) Hexachlorobenzene

57) Pentachlorophenol

58) Phenanthrene

59) Anthracene

60) Di-n-butylphthalate

61) Fluoranthene

62) *Chrysene-d12

63) Pyrene

64) \#Terphenyl-d16.

65) Butylbenzylphthalate

66) $3,3^{\prime}$-Dichlorobenzidine

67) Benzo(a) anthracene

68) Chrysene

69) Bis(2-Ethylhexyl)phthalate

70) *Perylene - d12

71) Di-n-octylphthalate

72) Benzo(b) fluoranthene

73) Benzo (k)fluoranthene

74) Benzo(a)pyrene

75) Indeno $(1,2,3$-cd)pyrene

76) Dibenz $(a, h)$ anthracene

77) Benzo( $(y, h, i)$ perylene

Step P2BN

$\frac{2 \mathrm{BN}}{90.5}$

0.0

85.1

86.8

97.8

84.3
113.8

100.0

0.0
105.2

$\begin{array}{r}0.0 \\ 89.8 \\ \hline 90.7\end{array}$

90.7

0.0

90.2

88.0

89.0

86.8

100.0

86.8

77.6

86.5

86.1

87.6

85.1

82.6

100.0

83.0

102.3

108.2

87.3

97.0

84.3

96.0

P2A

Total

NPH (Paraffin Hydrocarbon, C12-C14)

71.1

28.3

* Compound is an Internal Standard

\# Compound is a Surrogate Spike 
IABLE 12. Percent Recovery Results for Blank Spike, EPA 3630 Cleanup Method, Followed by Methylene Chloride Elution

\begin{tabular}{|c|c|c|c|c|c|c|}
\hline $\begin{array}{l}\text { Sample: EPA Blank Splke NPH (control) } \\
\text { No. Compound Name }\end{array}$ & E2N & E2A & $\mathrm{E} 2 \mathrm{~B}$ & E3N & E3N2 & $\begin{array}{r}\text { Total } \\
\text { Recovery }\end{array}$ \\
\hline 1) 1,4 -Dlchlorobenzene-d4 & 100.0 & 100,0 & 100.0 & 100.0 & 100.0 & 100.0 \\
\hline 2) \#2-Fluorophenol & 0.2 & 75.7 & & & & 75.7 \\
\hline 3) \#Phenol-d5 & 3.0 & 73.5 & 0.3 & & & $73, \overline{8}$ \\
\hline 4) Phenol & 2.8 & 64.8 & 4.3 & & & 69.1 \\
\hline 5) bis $(2$-Chloroethyl)ether & 55.2 & 0.4 & & & 9.2 & 9.6 \\
\hline 6) 2-Chlorophenol & 0.7 & 70.6 & & & & 70.6 \\
\hline 7) 1,3-Dlohlorobenzene & 55,0 & 0.4 & 4.2 & 0.3 & & 4.9 \\
\hline 8) 1,4-Dichlorobenzene & 54.2 & & 4.5 & 0.4 & & 4.8 \\
\hline 9) Benzyl alcohol & 54,3 & 1.8 & 7.8 & & & 9.6 \\
\hline 10) 1,2-Dlchlorobenzene & 53.2 & 0.4 & 4.4 & 1.3 & & 6.1 \\
\hline 11) 2-Mettiylphenol & 18.4 & 53.1 & 1.5 & & & 54,6 \\
\hline 12) bls(2-Chlorolsopropyl)ether & 65.8 & 0.5 & 5.1 & & 14.0 & 19.6 \\
\hline 13) 4-Mathylphenol & 14.1 & 57.6 & 9.1 & & & 58.7 \\
\hline 14) $N$-Nitroso-dl-n-propylamine & 62.0 & 0.7 & 4.5 & & & 5.2 \\
\hline 15) Hexachloroethane & 54.5 & 0.3 & 3.8 & 0.1 & & 4.2 \\
\hline 16) Naphthalene-d8 & 100.0 & 100.0 & 100.0 & 100.0 & 100.0 & 100.0 \\
\hline 17) \#Nitrobenzene-d5 & 61.2 & 0.4 & 5.0 & 5.4 & 8.7 & 19.5 \\
\hline 18) Nitrobenzeno & 61.8 & 0.5 & 5.3 & 7.0 & 9.4 & 22.1 \\
\hline 19) Isophorone & 59.5 & 1.3 & 5.0 & & & 6.3 \\
\hline 20) 2-Nitrophenol & 0.3 & 84.1 & & & & 84.1 \\
\hline 21) 2,4-Dlmethylphenol & 42.4 & 20.4 & 4,0 & 0.4 & & 24.7 \\
\hline 22) Benzolc acid & & 78.9 & & & & 78.9 \\
\hline 23) bls(2-Chloroethoxy) methane & 64.9 & 0.9 & 6.2 & & & 7.1 \\
\hline 24) 2,4-Dlchlorophenol & 0.6 & 82.3 & & & & 82.3 \\
\hline 25) 1,2,4-Trlchlorobenzene & 58.3 & 0.6 & 5.8 & 0.4 & & 6.8 \\
\hline 26) Naphthalene & 62.3 & 1.2 & 6.2 & 36.4 & & 43.8 \\
\hline 27) 4-Chloroanlline & 1.2 & & 87.6 & & & 87.6 \\
\hline 28) Hexachlorobutadlene & 57.5 & 0.5 & 5.4 & 0.3 & & 6.2 \\
\hline 29) 4-Chloro-3-methylphenol & 14.7 & 67.6 & 1.1 & & & 68.7 \\
\hline 30) 2-Methylnaphithalene & 63.0 & 0.8 & 6.6 & 40.2 & & 47.7 \\
\hline 31) "Acenaphthene-d10 & 100.0 & 100.0 & 100,0 & 100,0 & 100.0 & 100.0 \\
\hline 32) Hexachlorocyclopentadlene & 43.9 & & 1.8 & & & 1.8 \\
\hline 33) 2,4,6-Trichlorophenol & & 81.3 & & & & 81.3 \\
\hline 34) 2,4,5-Trichiorophenol & & 74.8 & & & & 74.8 \\
\hline 35) 2-Chloronaphthalene & 62.9 & 1.1 & 6.8 & 29.6 & & 37.4 \\
\hline 36) \#2-Fluoroblphenyl & 63,3 & 1.2 & 6.8 & 43.3 & & 51.3 \\
\hline 37) 2-Nitroanillne & 69.1 & 1.4 & 5.2 & & & 6.6 \\
\hline 38) Dimethylphthalate & 66.6 & 2.2 & 5.8 & & & 7.9 \\
\hline 39) Acenaphthylene & 63.0 & 1.2 & 6.3 & 44.6 & 0.0 & 52.1 \\
\hline 40) 2,6-Dinitrotoluene & 65,5 & 1.3 & 4.3 & & 15.2 & 20.8 \\
\hline 41) 3-Nitroanlline & 38.7 & & 91.6 & 0.3 & & 91.9 \\
\hline
\end{tabular}


IABIE 12. (contd)

\begin{tabular}{|c|c|c|c|c|c|c|}
\hline $\begin{array}{l}\text { Sample: EPA Blank Splke NPH (control) } \\
\text { No. Compound Name }\end{array}$ & E2N & E2A & E2B & E3N & E3N2 & $\begin{array}{r}\text { Total } \\
\text { Becovery }\end{array}$ \\
\hline 42) Acenaphthene & 68.6 & 1.5 & 7.0 & 48.9 & & 57.4 \\
\hline 43) 2,4-DInitrophenol & & 96.6 & & & & 96,6 \\
\hline 44) 4-Nitrophenol & & 85.5 & & & & 85.5 \\
\hline 45) Dlbenzofuran & 66.0 & 1.7 & 7.8 & 49.1 & & 58,6 \\
\hline 46) 2,4-Dinitrotoluene & 66.3 & 2.0 & 4,3 & & 9.8 & 16,2 \\
\hline 47) Dlethylphthalate & 70.8 & 2.3 & 5.7 & 0.2 & & 8,2 \\
\hline 48) 4-Chlorophenyl-phenylether & 42.2 & 1.2 & 4.6 & 35,9 & & 41.8 \\
\hline 49) Fluorene & 66.1 & 1.7 & 7.3 & 53.0 & 0.2 & 62.2 \\
\hline 50) 4-Nitroanilline & 52.1 & & 9.9 & & & 9.9 \\
\hline 51) *Pherianthrone-d10 & 100.0 & 100.0 & 100.0 & 100.0 & 100.0 & 100.0 \\
\hline 52) 4,6-Dinltro-2-methylphenol & & 97.3 & & & & 97.3 \\
\hline 53) $\mathrm{N}$-Nitrosodlphenylamine & 62.4 & 0.9 & 7.0 & 6.9 & 15.4 & 30.2 \\
\hline 54) \#2,4,6-Tribromophenol & & 92.1 & & & & 92.1 \\
\hline 55) 4-Bromophenyl-phenylether & 64.5 & 2.0 & $6, \overline{9}$ & 58,3 & 0.4 & 67.5 \\
\hline 56) Hexachlorobonzene & 64.7 & 2.2 & 6.0 & 0.8 & & 8.9 \\
\hline 57) Pentachlorophenol & & 88.5 & & & & 88.5 \\
\hline 58) Phenanthrene & 70.7 & 2.2 & 6.9 & 61.7 & 0.1 & 70.9 \\
\hline 59) Anthracene & 66.2 & 2.1 & 6.6 & 55.8 & 0.1 & 64.5 \\
\hline 60) Di-n-butylphithalate & 72.0 & 2.7 & 6.1 & 0.1 & 0.0 & 8,8 \\
\hline 61) Fluoranthene & 70.5 & 2.3 & 6.2 & 64.0 & 0.3 & 72.7 \\
\hline 62) *Chrysen $\theta-d 12$ & 100.0 & 100.0 & 100.0 & 100.0 & 100.0 & 100.0 \\
\hline 63) Pyrene & 73.6 & 2.4 & 5.9 & 61.7 & 0.3 & 70.2 \\
\hline 64) \#Terphenyl-d14 & 66.9 & 2.4 & 5.6 & 59.8 & 1.0 & 68.8 \\
\hline 65$)$ Butylbenzylphthalate & 75.7 & 3.0 & 5.9 & & & 8.9 \\
\hline 66) 3,3'-Dlchlorobenzidine & 29.4 & & 9.8 & & & 9.8 \\
\hline 67) Benzo(a)anthracene & 74.2 & 2.5 & 5.8 & 61.9 & 0.7 & 70.9 \\
\hline 68) Chrysene & 76.2 & 2.8 & 6.2 & 66.2 & 0.9 & 76.1 \\
\hline 69) Bis $(2-$ Ethylhexyl)phthalate & 72.8 & 3.0 & 5.9 & 1.2 & 0.1 & 10.2 \\
\hline 70) ॠPerylene-d12 & 100.0 & 100.0 & 100.0 & 100.0 & 100.0 & 100.0 \\
\hline 71) DI-n-octylphthalate & 68.1 & 2.7 & 5.7 & 1.1 & 0,0 & 9.5 \\
\hline 72) Benzo(b)fluoranthene & 73.3 & 2.2 & 5.6 & 57.6 & 0.9 & 66.3 \\
\hline 73) Benzo $(k)$ fluoranthene & 68.1 & 2.8 & 6.1 & 56.9 & 0.9 & 66.7 \\
\hline 74) Benzo(a)pyrene & 67.8 & 2.3 & 5,4 & 55.7 & 0.6 & 64.0 \\
\hline 75) Indeno $(1,2,3-c d)$ pyrene & 73.3 & 2.2 & 5.4 & 61.2 & 0.7 & 69.5 \\
\hline 76) Dibenz.(a,h)anthracene & 65.8 & 2.0 & 4.7 & 58.1 & 0.9 & 65.7 \\
\hline 77) Benzo $(g, h, l)$ perylene & 74.1 & 2.0 & 5.1 & 51.2 & 0.6 & 58.9 \\
\hline
\end{tabular}

\begin{tabular}{|l|l|l|l|l|}
\hline NPH (Paraffin Hydrocarbon, $\mathrm{C12}-\mathrm{C14})$ & & & & \\
\hline
\end{tabular}

- Compound is an Internal Standard

\# Compound is a Surrogate Splke 
TABLE 13. Percent Recovery Results for Blank Sptke with NPH, EPA 3630 Cleanup Method, Followed by Methylene Chloride Elution

\begin{tabular}{|c|c|c|c|c|c|c|}
\hline $\begin{array}{l}\text { Sample: EPA Blank Splke + NPH } \\
\text { No. Compound Name }\end{array}$ & E2N & E2A & $\mathrm{E2B}$ & E3N & E3N2 & $\begin{array}{r}\text { Total } \\
\text { Reoovery }\end{array}$ \\
\hline 1) ${ }^{* 1,4-D l o h l o r o b e n z e n \theta-d 4 ~}$ & 100,0 & 100.0 & 100.0 & 100.0 & 100,0 & 100.0 \\
\hline 2) \#2-Fluorophenol & 1.6 & 68.1 & & & & 68.1 \\
\hline 3) \#Phenol-d5 & 1.6 & 68.8 & & & & 68.8 \\
\hline 4) Phenol & 1.6 & 61.2 & & & & 61.2 \\
\hline 5) bls $(2-$ Chloroethyl)ether & 44.7 & 0.5 & 34.9 & & & 35,4 \\
\hline 6) 2-Chlorophenol & 0.5 & 65.2 & & & & 65.2 \\
\hline 7) 1,3-Dlohlorobenzene & 59.3 & 0.5 & 0,8 & & & 1.3 \\
\hline 8) 1,4-Dichlorobenzene & 62.1 & 0.5 & 0.9 & & & 1.5 \\
\hline 9) Benzyl alcohol & 57.3 & 2.9 & 3.0 & & & 5.9 \\
\hline 10) 1,2-Dlchlorobenzene & 58.3 & 0.5 & 1.0 & & & 1.5 \\
\hline 11) 2-Methylphenol & 10.9 & 55.9 & 0.3 & & & 56,1 \\
\hline 12) bls(2-Chlorolsopropyl)ettier & $74 . \overline{1}$ & 0.8 & 1.4 & & 4.1 & 6,4 \\
\hline 13) 4-Methylphenol & 7.7 & 58.7 & 0.2 & & & 58.8 \\
\hline 14) $\mathrm{N}$-Nitrosc-di-n-propylaming & 64.7 & 1.3 & 1.3 & & & 2.6 \\
\hline 15) Haxachloraethane & 58.0 & 0.4 & 0.8 & & & 1.2 \\
\hline 16) "Naphthalene-d8 & 100.0 & 100,0 & 100.0 & 100.0 & 100.0 & 100.0 \\
\hline 17) \#Nitrobenzene-dS & 61.9 & 0.7 & 1.4 & 5.8 & 23.1 & 31.0 \\
\hline 18) Nitrobenzene & 61.2 & 0.8 & 1.5 & 7.4 & 24.0 & 33.7 \\
\hline 19) Isophorone & 56.3 & 2.1 & $1 . \overline{6}$ & & & 3.7 \\
\hline 20) 2-Nitrophenol & & 72.6 & & & & 72.6 \\
\hline 21) 2,4-Dimethylphenol & 30.8 & 33.6 & 1.1 & 0.1 & & 34.9 \\
\hline 22) Benzolo acld & & 65,6 & & & & 65,6 \\
\hline 23) bis(2-Chloroothoxy)methane & $62 . \overline{8}$ & 1.7 & 2.6 & & & 4.3 \\
\hline 24) 2,4-Dlchlorophenol & 0.4 & 73.0 & & & & 73.0 \\
\hline 25) 1,2,4-Trlchlorobenzene & 59.7 & 1.0 & 2.6 & & & 3.6 \\
\hline 26) Naphthalene & 59.7 & 1.2 & 2.6 & 21.3 & & 25.1 \\
\hline 27) 4-Chloroanillne & & 1.3 & 82.5 & & & 83.8 \\
\hline 28) Hexachlorobutadlene & 57.7 & 0.9 & 2.1 & & & 3,0 \\
\hline 29) 4-Chloro-3-methylphenol & 7.4 & 70,4 & 0.3 & & & 70.7 \\
\hline 30) 2-Methyinaphthalene & 59.2 & 1,9 & 4.4 & 23.9 & & 30.2 \\
\hline 31) "Acenaphthene-d10 & 100.0 & 100.0 & 100.0 & 100.0 & 100.0 & 100.0 \\
\hline 32) Hexachlorocyclopentadiene & 50.0 & 0.5 & 0.7 & & & 1.2 \\
\hline 33) 2,4,6-Trichlorophenol & & 74.7 & & & & 74.7 \\
\hline 34) $2,4,5$-Trlchlorophenol & & 68.3 & & & & 68.3 \\
\hline 35) 2-Chloronaphthalene & 60.7 & 2.0 & 5.4 & 16.4 & & 23.8 \\
\hline 36) \#2-Fluoroblptienyl & 62.5 & 2.2 & 5.6 & 27.5 & & 35.2 \\
\hline 37) 2-Nitroanilline & 68.8 & 2.3 & 3.6 & & & 5.9 \\
\hline 38) Dirnethylphthalate & 65.3 & 3.1 & 3.7 & & & 6.7 \\
\hline 39) Acenaphthylene & 62.6 & 2.2 & 5,2 & 30,4 & & 37.8 \\
\hline 40) 2,6-Dinitrotoluene & 64.0 & 1.8 & 2.7 & & & 4.5 \\
\hline 41) $3-$ Nitroanillne & 100.0 & & 84.1 & & & 84.1 \\
\hline
\end{tabular}


IABLE 13. (contd)

Sample: EPA Blank Splke + NPH

No. Compound Name

42) Aoenaphthene

43) 2,4-Dinitrophenol

44) 4-Nitrophenol

45) Dibenzofuran

46 2,4-Dinitrotoluene

47) Dlethyiphthalate

48) 4-Chlorophenyl-phenylother

$49)$ Fluorene

50) 4-Nitroanillne

51) "phenanthrene-d10

52) 4,6-DInilio-2-methylphono

53) $N-N i t r o s o d i p h e n y l a m i n e$

54) \#2,4,6-7rlbromopherio

55) 4-Bromophenyl-phenylether

56) Hexachlorobenzene

57) Pentachlorophenol

58) Phenanthrene

59) Anthracene

$60)$ DI $-n-$ butylphthalate

61) Fluoranthene

62) Chrysene-d12

63) Pyrene

64 \#Terphenyl-d14

$65)$ Butylbenzylphthalato

66 3,3'-Dlchlorobenzldine

67) Benzo(a)anthracene

68) Chrysone

69) Bis(2-Ethylhexyl)phthalate

70 \#perylene-d12

71) DI-ri-octylphthalate

72) Benzo(b) fluoranthene

73) Benzo (k)fluoranthene

74) Benzo(a)pyrene

$75)$ Indeno $1,2,3$-cd)pyrene

76) Dibenz $(a, h)$ anthracene

77) Benzo $(g, h, 1)$ perylerio

\begin{tabular}{|c|c|c|c|c|c|}
\hline E2N & $E 2 A$ & $E_{22} B$ & $E 3 N$ & EON2 & $\begin{array}{l}\text { Total } \\
\text { Reoovery }\end{array}$ \\
\hline 66.1 & 2,6 & 5.9 & 35.7 & & 44,1 \\
\hline & 86,3 & & & & 86,3 \\
\hline & 77.0 & & & & 77.0 \\
\hline 65.9 & 2.7 & 7.7 & 39.5 & & 49.9 \\
\hline 66,3 & 2.7 & 2.7 & & & 5,4 \\
\hline 69.8 & 3,2 & 3.4 & & & 6.6 \\
\hline & & & 0.6 & & 0,6 \\
\hline 67.3 & 2,8 & 7.3 & 44,9 & & 65.0 \\
\hline 74 ! & 7.1 & 6.2 & & & 7.3 \\
\hline 100,0 & 100,0 & 100,0 & 100.0 & 100,0 & 100,0 \\
\hline & 86.9 & & & & 86,9 \\
\hline 66,7 & 1.4 & 6.5 & 12.2 & 40.9 & 61,0 \\
\hline & 92.6 & & & & 92.6 \\
\hline 65.6 & 3,0 & 8.1 & 47.4 & & 58.6 \\
\hline 65.1 & 3,0 & 4,4 & & & 7.4 \\
\hline & 84.7 & & & & 84.7 \\
\hline 66.9 & 3.3 & 6.9 & 51.5 & & 61.7 \\
\hline 63,6 & 3,0 & 6.9 & 49.8 & & 59.7 \\
\hline 66.6 & 4.8 & 3.4 & 0.0 & & 8.2 \\
\hline 67.1 & 3,4 & 5.0 & 52.9 & & $61 \overline{2}$ \\
\hline 100.0 & 100.0 & 100.0 & 100.0 & 100,0 & 100.0 \\
\hline 68.6 & 3.1 & 4.5 & 51.2 & & 88.8 \\
\hline 60.8 & 3.1 & 4.0 & 45.4 & 0.1 & $52, \overline{6}$ \\
\hline 68.1 & 3.5 & 3.1 & & & $6, \overline{6}$ \\
\hline 34.9 & 1.1 & 5.9 & & & 7.0 \\
\hline 69,3 & 3,2 & 4.1 & 52.8 & & 601 \\
\hline 69.8 & 3.6 & 4.1 & 55.9 & & 63.6 \\
\hline 62.7 & 3.6 & 2.9 & 0.1 & & 6.6 \\
\hline 100.0 & 100.0 & 100.0 & 100,0 & 100.0 & 100,0 \\
\hline 60,0 & 3,5 & 2.7 & & & 6.2 \\
\hline 65.5 & 3,4 & 3.3 & 50.7 & & 57.3 \\
\hline 66.4 & 3.6 & 3.7 & 51.8 & & 59.0 \\
\hline 63.1 & 3.1 & 3.4 & 50.6 & & 57.1 \\
\hline 69.1 & 3.2 & 3.1 & 52.3 & & 58,5 \\
\hline 59.7 & 2.7 & 2.7 & 46.2 & & 61.6 \\
\hline 66.8 & 3.1 & 2.9 & 45.3 & & 51.2 \\
\hline
\end{tabular}

NPH (Paraff In Hydrocarbon, C12-C14)

* Compound is an Internal Standara

\# Compound is a Surrogate Splke 
IABLE 14. Percent Recovery Results for Blank Sptke Dupltcate with NPH, EPA 3630 Cleanup Method, Followed by Methylene Chiloride E1ution

\begin{tabular}{|c|c|c|c|c|c|c|}
\hline $\begin{array}{l}\text { Sample: EPA Blank Splke + NPH Duplioate } \\
\text { No. Compound Name }\end{array}$ & E2N & $E_{2 A}$ & $E 2 B$ & E3N & E3N2 & $\begin{array}{r}\text { Total } \\
\text { Fecovery }\end{array}$ \\
\hline 1) *1, 4-Dlohlorobanzene-d4 & 100,0 & 100.0 & 00.0 & 100,0 & 100.0 & 100,0 \\
\hline 2) \#2-Fluorophonol & 0.2 & 51,4 & & & & $5 \longdiv { 1 . 4 }$ \\
\hline 3 \#Phono-d6 & 1,5 & 64.9 & & & & 64,9 \\
\hline 4) Phenol & 1.5 & 80,8 & & & & 50.8 \\
\hline 5) ble(2-Chloroethyl)ether & 39.6 & 1.0 & 28.4 & 0.3 & & 29.7 \\
\hline 6) 2-Chloropheno & & 69.9 & & & & 51.0 \\
\hline 7) 13-Dlohlorobenzene & 51.5 & 0.8 & 0,5 & 0.8 & & 2.2 \\
\hline 8) 1,4-Dlofilorobenzene & 52.2 & 0,9 & 0.7 & 1.0 & & 2.6 \\
\hline 9) Benzylalcohol & 52.8 & 3,0 & 1.6 & & & 4.6 \\
\hline 10) 12 -Dlchiorobenzeno & 51.5 & 0.8 & 0.6 & 3.1 & & 4.6 \\
\hline 11) 2-Methylphanol & 9.0 & 44.5 & & & & 44.5 \\
\hline 12) bla(2-Chlorolsopropyl)ether & 66.3 & 1,4 & & 9.0 & 16.7 & 26.1 \\
\hline 13) 4-Methy phenol & 6,6 & 47.6 & & & & 47,6 \\
\hline 14$) \mathrm{N}-\mathrm{N}$ troso-dl-n-propylamino & 57.2 & & 0.6 & & & 0,6 \\
\hline 15 Hexachloroethane & $48, \overline{3}$ & 0.5 & 0,6 & & & 1.1 \\
\hline 16) Naphthalen $\theta-\mathrm{d} 8$ & 100.0 & 100.0 & 100.0 & 100.0 & 100,0 & 100,0 \\
\hline 17) \#Nitrobenzen $\theta-\mathrm{d} 5$ & 59.0 & 1.2 & 0.4 & 21.7 & 62,6 & 86.0 \\
\hline 18) Nitrobenzene & 59.3 & 1.4 & 0,5 & $2.4,6$ & 66.8 & 93.3 \\
\hline 19) Isophorone & 59.7 & 3.2 & 0.9 & & & 4.1 \\
\hline 20) 2-Nitrophenol & 0.2 & 56.6 & & & & 56.6 \\
\hline 21) 2,4-Dimathylphenol & 30.7 & 26.0 & 0.4 & 0.1 & & 26.6 \\
\hline 22) Bonzolc acld & & 22.7 & & & & 22.7 \\
\hline 23) bls(2-Chloroethoxy) methene & 65.5 & 2.3 & 0.9 & & & 3.3 \\
\hline 24) 2,4-Dlchlorophenol & 0.4 & 63.1 & & & & 63.1 \\
\hline 25) 1,2,4-Trichlorobenzene & 60.6 & 1.7 & 1.1 & 0.9 & & 3,7 \\
\hline 26) Naphithaleng & 63.0 & 1,9 & 1,1 & 43.7 & & 46.7 \\
\hline 27) 4-Chloloanillne & & 3.0 & 71.1 & & & 74.0 \\
\hline 28) Hexachlorobutadlene & 60.3 & 1.6 & $7 \overline{3}$ & & & 2.8 \\
\hline 29) 4-Chloro-3-methylphenol & 6.6 & 58.2 & 0.0 & & & 58.2 \\
\hline 30) 2-Methylnaphthalene & 65.2 & 2.4 & $\sqrt{.5}$ & 48.9 & & 52.8 \\
\hline 31) "Acenaphthene-dio & 100.0 & 100.0 & 100.0 & 100.0 & 100.0 & 100.0 \\
\hline 32) Hexachlorocyolopentadiene & 36.2 & 0.6 & 0.2 & & & 0.8 \\
\hline 33) 2,4,6-Trlchlorophenol & & 60.0 & & & & 60.0 \\
\hline 34) $2,4,5$-Trlchlorophenol & & 56.5 & & & & 56.5 \\
\hline 35) 2-Chloronaphthalene & 63.9 & 2.7 & 1.6 & 29.7 & & 33.9 \\
\hline 36) \#2-Fluoroblphenyl & 66.2 & 2.7 & 1.7 & 51.1 & & 55.5 \\
\hline 37) $2-$ Nitroanillne & 69.6 & 3.4 & 0.6 & & & 4.0 \\
\hline 38) Dimethylphthalate & 71.6 & 5.4 & 1.6 & & & 7.0 \\
\hline 39) Acenaphthylene & 67.2 & 3,0 & 1.7 & 52.4 & & 57.1 \\
\hline 40) 2,6 -Dinitrotoluene & 65.0 & 2.7 & 0.5 & 1.4 & & 4.6 \\
\hline 41) 3 -Nitroanilline & 97.4 & & 60.6 & 0.1 & & 60.7 \\
\hline
\end{tabular}


IABLE 1.4. (contd)

\begin{tabular}{|c|c|c|c|c|c|c|}
\hline $\begin{array}{l}\text { Sample: EPA Blarik Splke + NPH Duplloate } \\
\text { No, Compound Name }\end{array}$ & E2N & $E 2 A$ & $E 28$ & EON & E3N2 & $\begin{array}{r}\text { Total } \\
\text { Recovery }\end{array}$ \\
\hline 42) Acenaphinene & 70.4 & 3.7 & 2.3 & 57.5 & & 63,5 \\
\hline 43$) 2,4-D$ initrophienal & & 33,9 & & & & 33,9 \\
\hline 44) 4-Nitrophenol & & 53.9 & & & & 53.1 \\
\hline 45) Dibenzofuran & 69.0 & 4.0 & 2.0 & 55.2 & & 61.2 \\
\hline 46) 2,4 -Dinitirotolueng & 64.9 & 42 & 0.6 & & & $4 . \overline{8}$ \\
\hline 47 Dlathylphthalate & 72.5 & 5,7 & 1.5 & 0.0 & & 7.2 \\
\hline 18 4-Chloropheny-phenylether & & & & & & 0.0 \\
\hline 49) Fluorene & 71.4 & 4.3 & 2.2 & 66.5 & & 62.9 \\
\hline 50) 4 -Nitroanilline & 73,2 & 2,0 & 1.5 & & & 3.6 \\
\hline 51) Phenanthreme-d10 & 100.0 & 100,0 & 100.0 & 100,0 & 100.0 & 100,0 \\
\hline 52) 4,6-Dinitro-2-methylphanol & & 53.4 & & & & 53.4 \\
\hline 53) $N-N$ ltrosodlphenylamilno & 79.3 & 4,4 & 22 & 40.8 & 111.3 & 158.7 \\
\hline 54) $2,4,6$-Trlbromophenol & & 73,9 & & & & 73.9 \\
\hline 55) 4-Bromophenyl-phenylettior & 73,1 & 4.6 & 21 & 60,8 & & 67.5 \\
\hline 56) Hexachlorobonzeno & 74.5 & 5,3 & 2.1 & 0.3 & & 7.6 \\
\hline 57 Pentachlorophenol & & 63.0 & & & & 63.0 \\
\hline 58 Phenanthrene & 73.8 & 5.6 & 2.4 & 63.6 & & 71.6 \\
\hline 59) Anthracerie & 70.9 & 5.2 & 21 & 60.0 & & 67.3 \\
\hline 60) DI-n-butylphthalate & 76,4 & 6.5 & 1.5 & 0.0 & & 8.1 \\
\hline 61) Fluoranthene & 70.5 & 5.9 & 2.0 & 64.7 & & 72.6 \\
\hline (62) Chrysene-d12 & 100,0 & 100.0 & 100.0 & 100.0 & 100.0 & 100.0 \\
\hline 63) Pyrene & 70.5 & 5,3 & 1.8 & 64.0 & & 71.2 \\
\hline 64) \#-Terpheny-d14 & 64,4 & 5.5 & 1.9 & 59.5 & 0.5 & 67.4 \\
\hline 65) Butylbenzylphthalate & 70.7 & 5.9 & 1.2 & & & 7.2 \\
\hline 66) 3,3 -Dichlorobenzidine & 45.0 & & 3,2 & & & 3.2 \\
\hline 67) Benzo(a)anthraceno & 71.6 & 5.7 & 1.9 & 64.2 & & $71 . \overline{7}$ \\
\hline 68$)$ Chrysene & 73,9 & 6,4 & $1 . \overline{8}$ & 67.3 & & 75,5 \\
\hline 69 Bls(2-Ethylhexyl)phthalato & 69.6 & 6.2 & 1.3 & 0.1 & & 7.6 \\
\hline 70) Porylene-d12 & 100.0 & 100.0 & 100.0 & 100.0 & 100.0 & 100.0 \\
\hline 71) D|-n-octylphthalate & 70.8 & 6.1 & 1.0 & 0.0 & & 7.1 \\
\hline 72) Berizo(b)fluoranthiene & 72.0 & 5.7 & 1.4 & 58.2 & & 65.4 \\
\hline 73$)$ Benzo $(\mathrm{k})$ fluoranthene & 76.3 & 6,6 & 1.7 & 58.6 & & 66.9 \\
\hline 74) Benzo a)pyrene & 67.2 & 5.7 & 1.4 & 57.0 & & 64.0 \\
\hline 75) Indeno $(1,2,3-c 0)$ pyrene & 74.5 & 5.6 & 1.2 & 57.1 & & 63.9 \\
\hline 76) Dlbenz (a, h) anthracene & 67.2 & 4.9 & 1.0 & 51.8 & & 57.7 \\
\hline 77) Benzo(g h,l)perylene & 71.2 & 5.5 & 1,2 & 55.1 & & 61.8 \\
\hline NPH (Paraffln Hyarocarbon, C12-C14) & 57.6 & 2.5 & 2.3 & & & 4.8 \\
\hline
\end{tabular}

* Compound ls an Internal Standard

\# Compound is a Surrogato Splke 
TABLE 15. Results for Single Shell Tank B110 Using Inttial PNL Cleariup Method

\begin{tabular}{|c|c|c|c|c|c|}
\hline $\begin{array}{l}\text { Sample: Tank B110 } \\
\text { No. Compound Name }\end{array}$ & Step 1 & P2BN & $P 2 A$ & PBBN & $\begin{array}{r}\text { Total } \\
\text { Peoovery }\end{array}$ \\
\hline 1) *1,4-Dlohlorobenzene-d4 & 100.0 & 100.0 & 100.0 & 100.0 & 100.0 \\
\hline 2) \#2-Fluorophenol & 91,1 & 0.1 & 26.2 & & 26.2 \\
\hline 3) \#Phenol-d5 & 103.0 & 1.0 & 32.6 & & 32,6 \\
\hline 4) Phenol & & & & & \\
\hline 5) b $8(2-$ Chloroethyl)ether & & & & & \\
\hline 6) 2 -Chlorophenol & & & & & \\
\hline 7$)$ 1,3-Dichlorobenzene & & & & & \\
\hline 8) 1,4-Dlohlorobenzene & & & & & \\
\hline 9) Bonzyl alcohol & & & & & \\
\hline 10) 1,2 -Dlchlorobenzene & & & & & \\
\hline 11) 2-Methylphenol & & & & & \\
\hline 12) bls(2-Chlorolsopropyl)ether & & & & & \\
\hline 13) 4-Methylpheriol & & & & & \\
\hline 14) $N-N \mid$ troso-d]-n-propylamine & & & & & \\
\hline 15) Hexachioroethane & & & & & \\
\hline 16) "Naphthalene-d8 & 100.0 & 100.0 & 100.0 & 100.0 & 100.0 \\
\hline 17) \#itrobenzene-d5 & 88.4 & 58.9 & 0.3 & & 0.3 \\
\hline 18) Nitrobenzene & & & & & \\
\hline 19) Isophorone & & & & & \\
\hline 20) 2-Nitrophenol & & & & & \\
\hline 21) 2,4-Dimetrylphenol & & & & & \\
\hline 22.) Benzolo acld & & & & & \\
\hline 23) bls(2-Chloroethoxy)methane & & & & & \\
\hline 24) 2,4-Dichlorophenol & & & & & \\
\hline 25) 1,2,4-Trlchlorobenzene & & & & & \\
\hline 26) Naphthalene & & & & & \\
\hline 27) 4-Chloroanilline & & & & & \\
\hline 28) Hexachlorobutadlene & & & & & \\
\hline 29) 4-Chloro-3-methylphenol & & & & & \\
\hline 30) 2-Mlethylnaphthalene & & & & & \\
\hline 31) *Acenaphthene-d10 & 100.0 & 100.0 & 100.0 & 100.0 & 100.0 \\
\hline 32) Hexachlorocyolopentadieno & & & & & \\
\hline 33) 2,4,6-Trichloroprianol & & & & & \\
\hline 34) 2,4,5-Trichlorophenol & & & & & \\
\hline 35) 2-Chloronaphthalene & & & & & \\
\hline 36) \#2-Fluorobiphenyl & 96.2 & 59.6 & 0.8 & 55.4 & 56.2 \\
\hline 37) 2 -Nitroanilline & & & & & \\
\hline 38) Dimethylphthalate & & & & & \\
\hline 39) Acenaphithylene & & & & & \\
\hline 40) $2,6-$ Dinitrotoliene & & & & & \\
\hline 41) 3-Nitroanilline & & & & & \\
\hline
\end{tabular}


IABLE 15. (contd)

Sample: Tank B110

No. Compound Name

42) Acenaphthene

43) 2,4-Dinitrophenol

44) 4-Nitrophenol

45) Dibenzofuran

46) 2,4-Dinitrotoluene

47) Diethylphthalate

48) 4-Chlorophenyl-phenylether

49) Fluorene

50) 4-Nitroaniline

51) *Phenanthrene-d10

52) 4,6-Dinitro-2-rnethylphenol

53) $\mathrm{N}$-Nitrosodiphenylamine

54) \#2,4,6-Tribromophenol

55) 4-Bromophenyl-phenylether

56) Hexachlorobenzene

57) Pentachlorophenol

58) Phenanthrene

59) Anthracene

60) Di-n-butylphthalate

61) Fluoranthene

62) *Chrysene-d12

63) Pyrene

64) \#Terphenyl-d14

65) Butylbenzylphthalate

66) 3,3'-Dichlorobenzidine

67) Benzo(a)anthracene

68) Chrysene

69) Bis(2-Ethylhexyl)phthalate

70) *Perylene - d12

71) Di-n-octylphthalate

72) Benzo(b)fluoranthene

73) Benzo (k)fluoranthene

74) Benzo(a)pyrene

$75)$ Indeno $(1,2,3-\mathrm{co})$ pyrene

76) Dibenz $(a, h)$ anthracene

77) Benzo(g,h,i)perylene

NPH (Paraffin Hydrocarbon, C12-C14)

* Compound is an Internal Standard

\# Compound is a Surrogate Spike

Total Step 1 P2BN P2A P3BN Recover

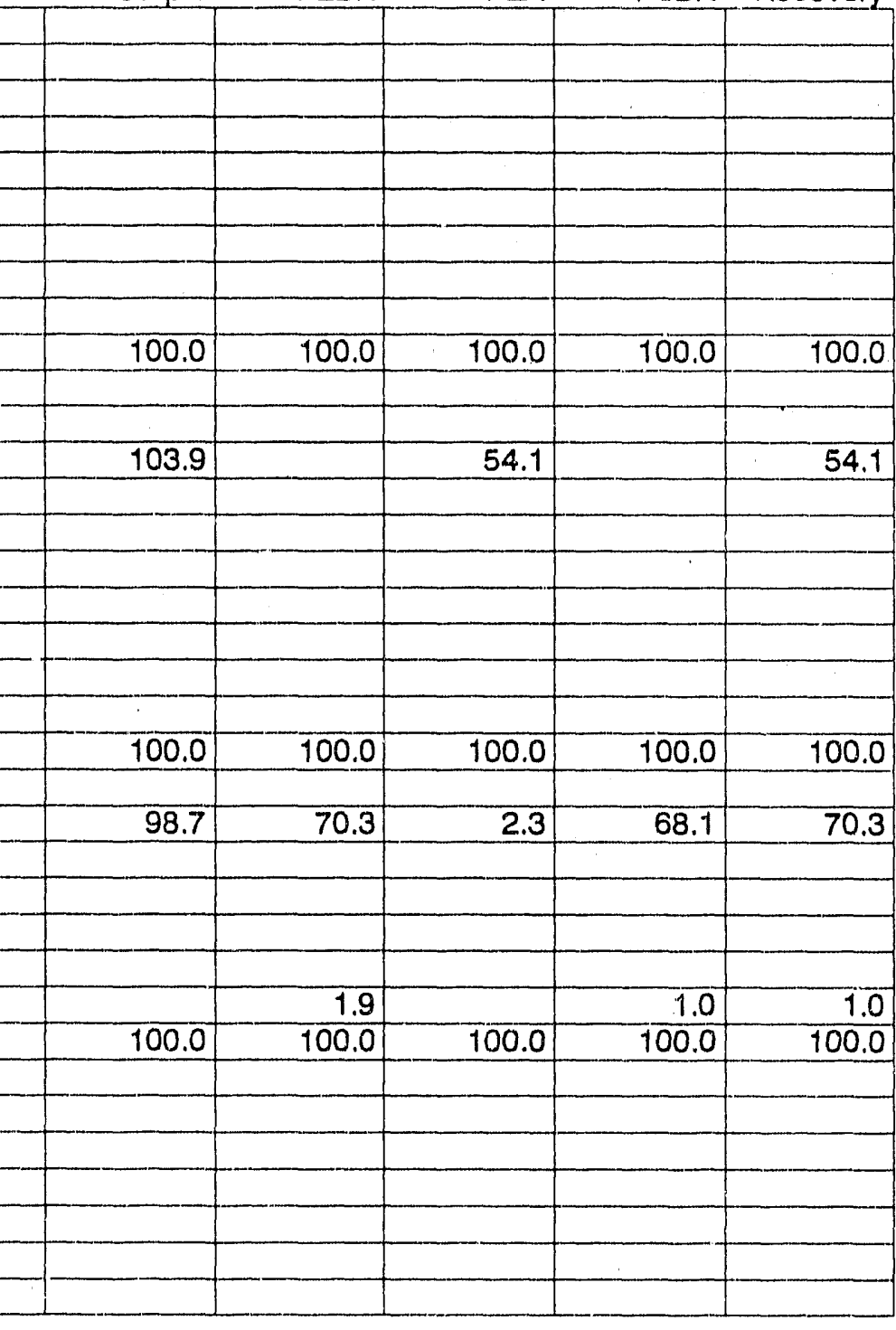

133.0

71.4

1.2

16.8

18.0 
TABLE 16. Percent Recovery Results for Single She11 Tank B110 Matrix Spike, Initial PNL Cleanup Method

Sample: Tank B110 Matrix Spike

No. Compound Name

1) *1,4-Dichlorobenzene-d4

2) \#2-Fluorophenol

3) \#Phenol-d5

4) Phenol

5) bis(2-Chloroethyl)ether

6) 2-Chlorophenol

7) 1,3-Dichlorobenzene

8) 1,4-Dichlorobenzene

9) Benzyl alcohol

10) 1,2-Dichlorobenzene

11) 2-Methylphenol

12) bis(2-Chloroisopropyl)ether

13) 4-Methylphenol

14) N-Nitroso-di-n-propylamine

15) Hexachloroethane

16) *Naphthalene-d8

17) \#Nitrobenzene-d5

18) Nitrobenzene

19) Isophorone

20) 2-Nitrophenol

21) 2,4-Dimethylphenol

22) Benzoic acid

23) bis(2-Chloroethoxy)methane

24) 2,4-Dichlorophenol

25) 1,2,4-Trichlorobenzene

26). Naphthalene

27) 4-Chloroaniline

28) Hexachlorobutadiene

29) 4-Chloro-3-methylphenol

30) 2-Methylnaphthalene

31) *Acenaphthene-d10

32) Hexachlorocyclopentadiene

33) 2,4,6-Trichlorophenol

34) 2,4,5-Trichlorophenol

35) 2--Chloronaphthalene

36) \#2-Fluorobiphenyl

37) 2-Nitroaniline

38) Dimethylphthalate

39) Acenaphthylene

40) 2,6-Dinitrotoluene

41) 3-Nitroaniline

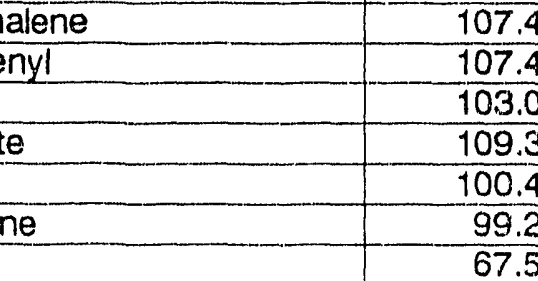

67.5

Step 1 P2BN

100.0

104.3

118.5

108.6

110.3

100.4

98.2

101.5

98.6

99.9

106.0

105.2

97.8

100.0

106.4

106.2

104.0

106.2

99.0

75.8

114.5

111.7

109.5

109.9

136.9

102.9

113.5
93.8

100.0

92.0

106.3

39.9

07.4

07.4

03.0

09.3

00.4

P2BN

$\frac{0.4}{3.6}$

3.6

3.1

59.6

0.8

52.3

51.8

60.5

52.2

20.3

68.4

15.9

65.4

52.4

60.1

56.7
56.5

0.3

40.2

61.0

01.0

0.7
55.8

54.9

56.9

15.3

41.0

100.0

50.2

$\begin{array}{r}\text { P2A } \\ 100.0 \\ \hline 42.6\end{array}$

45.5

39.9

39.9
0.3
43.1

0.2

0.2

0.2
27.8

0.3

0.3

0.2

0.2

0.5

0.4
50.6

0.9

0.9

39.7
0.4

\begin{tabular}{|r}
\hline \\
\hline \\
\hline
\end{tabular}

\begin{tabular}{|r|}
\hline 52.9 \\
\hline 0.3 \\
\hline 0.4
\end{tabular}

\begin{tabular}{|r|r|r|}
\hline 6 & & 50.6 \\
\hline .7 & 0.2 & 1.1 \\
\hline .4 & & 39.7 \\
\hline .3 & & 0.4 \\
\hline .4 & 46.9 & 52.9 \\
\hline .3 & 52.4 & 52.2 \\
\hline .6 & 18.8 & 19.1 \\
\hline .5 & 58.2 & 58.6 \\
\hline .1 & 100.0 & 100.0 \\
\hline .3 & 16.4 & 16.5 \\
\hline 8 & & 53.3 \\
\hline .4 & 61.1 & 51.8 \\
\hline .4 & 61.8 & 61.5 \\
\hline .4 & & 62.2 \\
\hline .6 & & 0.4 \\
\hline .4 & 60.9 & 61.3 \\
\hline .3 & & 0.3 \\
\hline & & 0.0 \\
\hline
\end{tabular}

\begin{tabular}{|r|r|r} 
& P3BN Recovery \\
\hline 100.0 & 100.0 \\
\hline
\end{tabular}

Total

$\begin{array}{r} \\ \hline 55.3 \\ \hline 58.7 \\ \hline 66.6 \\ \hline 61.7 \\ \hline 53.1 \\ \hline 63.5 \\ \hline 73 . \\ \hline\end{array}$

\begin{tabular}{r}
0.3 \\
42.6 \\
0.5 \\
100.0 \\
\hline 0.1 \\
53.3 \\
51.8 \\
0.4 \\
\hline 0.4 \\
\hline 0.4 \\
\hline 0.6 \\
\hline 0.4 \\
0.3
\end{tabular}

\begin{tabular}{r|r|}
\hline 0.0 & 42.6 \\
\hline & 45.5 \\
\hline & 39.9 \\
\hline & 0.3 \\
\hline
\end{tabular}

\begin{tabular}{r|r|}
\hline & 39.9 \\
\hline & 0.3 \\
\hline
\end{tabular}

\begin{tabular}{r|r|}
\hline 31.9 & 32.1 \\
\hline 35.6 & 35.8 \\
\hline
\end{tabular}

\begin{tabular}{r|r}
\hline 36.7 & 0.8 \\
\hline
\end{tabular}

\begin{tabular}{|r|r|}
\hline & 36.9 \\
\hline & 27.9 \\
\hline & 0.1. \\
\hline & 31.8 \\
\hline 13.5 & 0.3 \\
\hline
\end{tabular}

\begin{tabular}{r|r}
13.5 & 13.6 \\
\hline 100.0 & 100.0 \\
\hline
\end{tabular}

\begin{tabular}{|r|r|}
\hline 1.0 & 1.2 \\
\hline 1.4 & 1.9 \\
\hline & 0.4 \\
\hline
\end{tabular}


TABLE 16. (contd)

Sample: Tank B110 Matrix Spike

No. Compound Name

42) Acenaphthene

43) 2,4-Dinitrophenol

44) 4-Nitrophenol

45) Dibenzofuran

46) 2,4-Dinitrotoluene

47) Diethylphthalate

48) 4-Chlorophenyl-phenylether

49) Fluorene

50) 4-Nitroaniline

51) *Phenanthrene-d10

52) 4,6-Diritro-2-methylphenol.

53) $\mathrm{N}$-Nitrosodiphenylamine

54) \#2,4,6-Tribromophenol

55) 4-Bromophenyl--phenylether

56) Hexachlorobenzene

57) Pentachlorophenol

58) Phenanthrene

59) Anthracene

60) Di-n-butylp ithalate

61) Fluoranthene

62) *Chrysene-d12

63) Pyrene

64) \#Terphenyl-d14

65) Butylbenzylphthalate

66) 3,3'-Dichlorobenzidine

67) Benzo(a) anthracene

68) Chrysene

69) Bis (2-Ethylhexyl)phthalate

70) *Perylene-d12

71) Di-n-octylphthalate

72) Benzo(b) fluoranthene

73) Benzo (k)fluoranthene

74) Benzo (a) pyrene

75) Indeno (1,2,3-cd)pyrene

76) Dibenz $(a, h)$ anthracene

77) Benzo $(g, h, i)$ perylene

NPH (Paraffin Hydrocarbon, $\mathrm{C} 12-\mathrm{C} 14)$

- Compound is an Internal Standard

\# Compound is a Surrogate Spike

Step 1

115.1

96.9

107.6

101.4

111.1

69.0

108.4

151.1

100.0

78.7

129.2

102.6

105.3

103.7

89.2

109.9

103.1

107.7

102.6

100.0

107.3

98.2

112.9

78.2

108.0

114.4

116.6

100.0

110.3

108.2

103.2

102.5

107.5

97.5

103.6

168.8

74.7

0.6

4.0

4.6

P2BN P2A P3BN Recovery

\begin{tabular}{r|r|r|r|}
61.5 & 0.5 & 69.1 & 69.5 \\
\hline & 58.5 & & 58.5 \\
\hline
\end{tabular}

62

64.0

36.7

61.7
114.4

100.0

63.7

57.0

60.7

62.2

58.1

59.8

100.0

59.8

61.

25.6

60.5

56.4

100.0
54.7

63.2

48.5

$\frac{55.1}{62.0}$

56.1

60.4

55.5

0.5

0.5

0.3

0.2

100.0

0.

Total 
TABLE 17. Percent Recovery Results for Single She11 Tank B110 Matrix Spike Duplicate, Initial PNL Cleanup Method

Sample: Tank B110 Matrix Spike Duplicate

No. Compound Name

\begin{tabular}{|c|c|}
\hline No. Compound Name & Step 1 \\
\hline 1) *1,4-Dichlorobenzene-d4 & 100.0 \\
\hline 2) \#2-Fluorophenol & 106.3 \\
\hline 3) \#Phenol-d5 & 120.1 \\
\hline 4) Phenol & 111.8 \\
\hline 5) bis(2-Chloroethyl)ether & 111.5 \\
\hline 6) 2-Chlorophenol & 101.8 \\
\hline 7) 1,3-Dichlorobenzene & 101.3 \\
\hline 8) 1,4-Dichlorobenzene & 101.0 \\
\hline 9) Benzyl alcohol & 101.4 \\
\hline 10) 1,2-Dichlorobenzene & 102.0 \\
\hline 11) 2-Methylphenol & 98.7 \\
\hline 12) bis(2-Chloroisopropyl)ether & 115.7 \\
\hline 13) 4-Methylphenol & 102.3 \\
\hline 14) $N$-Nitroso-di-n-propylamine & 103.2 \\
\hline 15) Hexachloroethane & 101.8 \\
\hline 16) *Naphthalene-d8 & 100.0 \\
\hline 17) \#Nitrobenzene-d5 & 104.2 \\
\hline 18) Nitrobenzene & 107.9 \\
\hline 19) Isophorone & 107.1 \\
\hline 20) 2-Nitrophenol & 102.9 \\
\hline 21) 2,4-Dimethylphenol & 92.7 \\
\hline 22) Benzoic acid & 83.1 \\
\hline 23) bis $(2-$ Chloroethoxy) methane & 118.3 \\
\hline 24) 2,4-Dichlorophenol & 112.7 \\
\hline 25) 1,2,4-Trichlorobenzene & 110.7 \\
\hline 26) Naphthalene & 112.1 \\
\hline 27) 4-Chloroaniline & 127.9 \\
\hline 28) Hexachlorobutadiene & 108.8 \\
\hline 29) 4-Chloro-3-methylphenol & 114.6 \\
\hline 30) 2-Methylnaphthalene & 97.2 \\
\hline 31) *Acenaphthene-d10 & 100.0 \\
\hline 32) Hexachlorocyclopentadiene & 83.7 \\
\hline 33) 2,4,6-Trichlorophienol & 104.7 \\
\hline 34) 2,4,5-Trichlorophenol & 96.6 \\
\hline 35) 2-Chloronaphthalene & 104.6 \\
\hline 36) \#2-Fluorobiphenyl & 106.1 \\
\hline 37) 2-Nitroaniline & 98.8 \\
\hline 38) Dimethylphthalate & 104.6 \\
\hline 39) Acenaphthylene & 94.9 \\
\hline 40) 2,6 -Dinitrotoluene & 95.0 \\
\hline 41) 3-Nitroaniline & 61.9 \\
\hline
\end{tabular}

P2BN

100.0

\begin{tabular}{|r|}
0.5 \\
\hline 4.4 \\
\hline
\end{tabular}

5

4.3

77.1

77.1

68.3

69.5

69.5

69.7

24.5

19.4

91.7

69.5
100.0

81.4

81.0

0.4

49.7

22) Benzoic acid

3) bis(2-Chloroethoxy)methane

84.8

0.9

74.5

74.4

100.0

28) Hexachlorobutadiene

29) 4-Chloro-3-mettylphenol

77.3

$\frac{77.3}{18.8}$

54.6

100.0

62.9

32) Hexachlorocyclopentadiene

34) 2,4,5-Trichlorophenol

35) 2-Chloronaphthalene

77

77.0

78.0

83.2

69.9

85.8

100.0

P2A

Total

P2A P3BN Recovery

\begin{tabular}{|r|r|r|}
\hline 57.3 & 0.0 & 57.3 \\
\hline
\end{tabular}

61.7
57.6

\begin{tabular}{|r|r|}
\hline 57.6 & 57.6 \\
\hline 0.6 &
\end{tabular}

58.7

\begin{tabular}{r|}
58.7 \\
\hline 0.4
\end{tabular}

0.4

$-\frac{1.9}{0.4}$

\begin{tabular}{r|}
0.4 \\
.9 \\
0.4 \\
\hline 4.3
\end{tabular}

0.7

50.8

0.8

10

0.3

\begin{tabular}{rr|r}
0.17 .8 & 18.2 \\
\hline 100.0 & 100.0
\end{tabular}

\begin{tabular}{r|r|r}
0.0 & 100.0 & 100.0 \\
\hline 0.5 & & 0.5
\end{tabular}

\begin{tabular}{|r|r|r|}
\hline 1.2 & & 1.2 \\
\hline 71.0 & & 71.0 \\
\hline 9.6 & 0.2 & 9.8 \\
\hline 56.6 & & 56.6 \\
\hline 1.1 & & 1.1 \\
\hline 73.2 & & 73.2 \\
\hline 0.7 & 60.1 & 60.8 \\
\hline 1.1 & 63.2 & 64.3 \\
\hline 0.1 & & 0.1 \\
\hline 0.6 & 25.5 & 26.2 \\
\hline 63.5 & & 63.5 \\
\hline 1.1 & 61.5 & 62.6 \\
\hline 100.0 & 100.0 & 100.0 \\
\hline 0.3 & 13.3 & 13.5 \\
\hline 73.3 & & 78.3 \\
\hline 75.7 & & 75.7 \\
\hline 1.1 & 67.1 & 68.2 \\
\hline 1.1 & 66.3 & 67.5 \\
\hline 1.4 & & 1.4 \\
\hline 2.1 & & 2.1 \\
\hline 1.1 & 62.8 & 63.9 \\
\hline 1.1 & & 1.1 \\
\hline 0.6 & 0.1 & 0.7 \\
\hline & & \\
\hline
\end{tabular}

\begin{tabular}{r|r}
0.6 & 0.6 \\
\hline 12 & 12 \\
\hline
\end{tabular}


IABLE 17. (contd)

Sample: Tank B110 Matrix Spike Duplicate

No. Compound Name

42) Acenaphthene

43) 2,4-Dinitrophenol

44) 4-Nitrophenol

45) Dibenzofuran

46) 2,4-Dinitrotoluene

47) Diethyiphthalate

48) 4-Chlorophenyl-phenylether

49) Fluorene

50) 4-Nitroaniline

51) *Phenanthrene-d10

52) 4,6-Diniltro-2-methylphenol

53) $\mathrm{N}$-Nitrosodiphenylamine

54) \#2,4,6-Tribromophenol

55) 4-Bromophenyl-phenylether

56) Hexachlorobenzene

57) Pentachlorophenol

58) Phenanthrene

59) Anthracene

60) Di-n-butylphthalate

61) Fluoranthene

62) *Chrysene $-d 12$

63) Pyrene

64) \#Terphenyl-d14

65) Butylbenzylphthalate

66) $3,3^{\prime}$-Dichlorobenzidine

67) Benzo (a) anthracene

68) Chrysene

69) Bis(2-Ethylhexyl)phthalate

70) *Perylene-d12

71) Di-n-octylphthalate

72) Benzo(b) fluorantherie

73) Benzo(k)fluoranthene

74) Benzo(a)pyrene

75) indeno (1,2,3-cd)pyrene

76) Dibenz $(a, h)$ anthracene

77) Benzo(g,h,i)perylene

\begin{tabular}{|c|}
- \\
\hline
\end{tabular}

Step $1 \quad$ P2BN

Total

P2A PBBN Recovery

58.2

88.6

93.5

106.8

65.9
105.9

134.1

$\frac{100.0}{77.6}$

126.9

98.3

105.5

100.5

87.3

110.1

100.5

107.5

103.6

100.0

104.3

96.4

105.1

66.2

104.4

112.7

110.1

100.0

109.6

105.1

102.3

98.3

105.4

99.6

106.9

86.1

1

1.4
77.2
82.1

84.4

87.8

90.6

49.3

49.3

156.0

100.0

NPH (Paraffin Hydrocarbon, C12-C14)

192.7

87.8

87.8

78.6
85.8

85

85.2

85.5

82.3

100.0

80.1

73.1

80.8
100.0

\begin{tabular}{r}
81.5 \\
\hline 82.6 \\
74.5
\end{tabular}

74.5

\begin{tabular}{l}
77.8 \\
87.3 \\
\hline 87.5
\end{tabular}

87.5
76.5

87.3

78.8

85.7

82.1

1.6
1.8

2.4

$\frac{1.1}{1.8}$

1.8

100.0

85.5

$\begin{array}{r}1.4 \\ 81.6 \\ \hline\end{array}$

1.9

2.0

\begin{tabular}{|r}
\hline \\
\hline
\end{tabular}

$$
2.0
$$

1.6

2.6

2.1
100.0

2.

2.0

2.4

* Compound is an Internal Standard

\# Compound is a Surrogate Spike 


\section{TABLE 18. Results for Single She11 Tank U110 Using Initial PNL Cleanup Method}

\begin{tabular}{|c|c|c|c|c|c|c|}
\hline \multicolumn{7}{|l|}{$\begin{array}{l}\text { Sample: Tank U110 } \\
\text { No. Compound Name }\end{array}$} \\
\hline 1) $\star 1,4$-Dichlorobenzens-d4 & 100.0 & 100.0 & 100.0 & 100.0 & 100.0 & 100.0 \\
\hline 2) \#2-Fluorophenol & 94.7 & 98.3 & & 56.0 & & 56.0 \\
\hline 3) \#Phenol-d5 & 109.9 & 106.2 & 1.0 & 62.7 & & 62.7 \\
\hline \multicolumn{7}{|l|}{ 4) Phenol } \\
\hline \multicolumn{7}{|l|}{ 5) bis(2-Chloroethyl)ether } \\
\hline \multicolumn{7}{|l|}{ 6) 2-Chlorophenol } \\
\hline \multicolumn{7}{|l|}{ 7) 1,3-Dlchlorobenzene } \\
\hline \multicolumn{7}{|l|}{ 8) 1,4-Dichlorobenzene } \\
\hline \multicolumn{7}{|l|}{ 9) Benzyl alcohol } \\
\hline \multicolumn{7}{|l|}{ 10) 1,2-Dichlorobenzene } \\
\hline \multicolumn{7}{|l|}{ 11) 2-Methylphenol } \\
\hline \multicolumn{7}{|l|}{ 12) bls(2-Chlorolsopropyl)ether } \\
\hline \multicolumn{7}{|l|}{ 13) 4-Methylphenol } \\
\hline \multirow{2}{*}{\multicolumn{7}{|c|}{$\begin{array}{l}\text { 14) } N \text {-Nitroso-di-n-propylamine } \\
\text { 15) Hexachloroethane }\end{array}$}} \\
\hline & \multirow{2}{*}{\multicolumn{6}{|c|}{ 15) Hexachloroethane }} \\
\hline 16) Naphthalene-d8 & 100.0 & 100.0 & 100.0 & & & 100.0 \\
\hline 17) \#Nitrobenzene-d5 & 89.7 & 89.5 & 58.5 & 0.2 & 3.7 & 3.9 \\
\hline \multicolumn{7}{|l|}{ 18) Nitrobenzene } \\
\hline \multicolumn{7}{|l|}{ 19) Isophorone } \\
\hline \multicolumn{7}{|l|}{ 20) 2-Nitrophenol } \\
\hline \multicolumn{7}{|l|}{ 21) 2,4-Dimethylphenol } \\
\hline \multicolumn{7}{|l|}{ 22) Benzolc acld } \\
\hline \multicolumn{7}{|l|}{ 23) bis(2-Chloroethoxy)methan } \\
\hline \multicolumn{7}{|l|}{ 24) 2,4-Dichlorophenol } \\
\hline \multirow{2}{*}{\multicolumn{7}{|c|}{$\begin{array}{l}\text { 25) 1,2,4-Trichlorobenzene } \\
\text { 26) Naphthalene }\end{array}$}} \\
\hline & & & & & & \\
\hline \multicolumn{7}{|l|}{ 27) 4 -Chloroanlline } \\
\hline \multicolumn{7}{|l|}{ 28) Hexachiorobutadiene } \\
\hline \multirow{2}{*}{\multicolumn{7}{|c|}{$\begin{array}{l}\text { 29) 4-Chloro-3-methylphenol } \\
\text { 30) 2-Methylnaphthalerie }\end{array}$}} \\
\hline & & & & \multicolumn{3}{|c|}{ 30) 2-Methylnaphthalerie } \\
\hline 31) Acenaphthene-d10 & 100.0 & 100.0 & 100.0 & 100.0 & 100.0 & 100.0 \\
\hline 32) Hexachlorocyclopentadiene & & & & & & \\
\hline 33) 2,4,6-Trichlorophenol & & & & & & \\
\hline 34) 2,4,5-Trichlorophenol & & & & & & \\
\hline 35) 2-Chloronaphthalene & & & & & & \\
\hline 36) \#2-Fluorobiphenyl & 95.4 & 89.2 & $60 . \overline{7}$ & 0.5 & 57.2 & 57.7 \\
\hline 37) 2 -Nitroaniline & & & & & & \\
\hline 38) Dimethylphthalate & & & & & & \\
\hline 39) Acenaphthyiene & & & & & & \\
\hline 40) 2,6-Dinitrotoluene & & & & & & \\
\hline 41) 3-Nitroaniline & & & & & & \\
\hline
\end{tabular}


TABLE 18. (contd)

Sample: Tank U110

No. Compound Name

42.) Acenaphthene

43) 2,4-Dinitropheno

44) 4-Nitrophenol

$45)$ Dibenzofuran

46) 2,4-Dinitrotoluen $\theta$

47) Dlethylphthalate

48) 4-Chlorophenyl-phenylether

49) Fluorene

50) 4-Nitroanilline

51) *Phenanthrene-d10

52) 4,6-DInitro-2-methylpheno

53) $\mathrm{N}$-Nitrosodiphenylamine

54) \#2,4,6-Trlbromophenol

55) 4-Bromophenyl-phenylether

56) Hexachloroberizene

57) Pentachlorophenol

58) Phenanthrene

59) Arthracone

60) DI-n-butylphthalate

61) Fluorantherie

62) *Chrysen $\theta-\mathrm{d} 12$

63) Pyrene

64) \#Terphenyl-d14

65) Butylbenzylphthalate

66) 3,3'-Dichlorobenzidine

67) Benzo(a)anthracene

68) Chrysene

69) Bls(2-Ethylhexyl)phthalate

70) *Perylene-d12

71) DI-n-octylphthalate

72) Benzo(b)fluoranthene

73) Benzo(k)fluoranthene

74) Benzo(a)pyrene

75) Indeno (1,2,3-cd)pyrene

76) Dibenz $(a, h)$ anthracene

77) Benzo $(g, h, i)$ perylene

Step 1 Re-Step $1 \quad$ P2BN

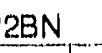

P2A

Total

ecovery

NNPH (Paraffin Hydrocarbon, C12-C14)

* Compound is an Internal Standara

\# Compound is a Surrogate Spike 
TABLE 19. Percent Recovery Results for Single Shell Tank U110 Matrix Spike, Initial PNL Cleanup Method

\begin{tabular}{|c|c|c|c|c|c|c|}
\hline $\begin{array}{l}\text { Sample: Tank U110 Matrlx Splke } \\
\text { No. Compound Name }\end{array}$ & Step 1 & Step 1 & P2BN & P2A & P3BN & $\begin{array}{r}\text { Total } \\
\text { Recovery }\end{array}$ \\
\hline 1) *1,4-Dlchlorobenzene-d4 & 100.0 & 100.0 & 100.0 & 100.0 & 100.0 & 100.0 \\
\hline 2) \#2-Fluorophenol & 107.7 & 110.9 & 0.2 & 50.7 & 0.0 & 50.7 \\
\hline 3) \#Phenol-d5 & 117.9 & 121.6 & 2.2 & 57.7 & & 57.7 \\
\hline 4) Phenol & 114.6 & 104.7 & 3.7 & 53.7 & & 5.3 .7 \\
\hline 5) bls(2-Chloroethyl)ether & 89.7 & 121.4 & 60.8 & 0.7 & & 0.7 \\
\hline 6) 2-Chlorophenol & 96.6 & 101.7 & 0.4 & 48.4 & & 48.4 \\
\hline 7) 1,3-Dlchlorobenzene & 102.7 & 101.9 & 57.7 & 0.5 & 1.1 & 1.6 \\
\hline 8) 1,4-Dlchlorobenzene & 107.0 & 104.4 & 57.0 & 0.5 & 2.4 & 2.9 \\
\hline 9) Bonzylalcohol & 105.6 & 120.2 & 64.1 & 2.4 & & 2.4 \\
\hline 10) 1,2-Dlchlorobenzene & 98.7 & 100.9 & 57.6 & 0.5 & 16.3 & 16.8 \\
\hline 11) 2-Methylphenol & 91.9 & 97.7 & 11.7 & 40.5 & & 40.5 \\
\hline 12) bis(2-Chlorolsopropyl)ether & 116.8 & 131.8 & 83.2 & 0.9 & & 0.9 \\
\hline 13) 4-Methylphenol & 102.9 & 108.0 & 9.3 & 47.3 & & 47.3 \\
\hline 14) $N-N \mid t r o s o-d i-n-p r o p y \operatorname{lam} \ln \theta$ & 118.4 & 138.8 & 72.0 & 1.7 & & 1.7 \\
\hline 15) Hexachloroethane & 100.2 & 105.2 & 57.2 & 0.4 & & 0.4 \\
\hline 16) *Naphthalene-d8 & 100.0 & 100.0 & 100.0 & 100.0 & 100.0 & 100.0 \\
\hline 17) \#Nitrobenzene-d5 & 103.3 & 108.9 & 66.3 & 0.7 & & 0.7 \\
\hline 18) Nitrobenzene & 106.9 & 109.2 & 68.5 & 1.1 & & 1.1 \\
\hline 19) Isophorone & 104.5 & 108.8 & 61.5 & 2.4 & & 2.4 \\
\hline 20) 2-Nitrophenol & 112.3 & 108.9 & 0.2 & 62.0 & & 62.0 \\
\hline 21) 2,4-Dimethylphenol & 57.7 & 56.3 & 15.8 & 7.7 & 0.1 & 7.8 \\
\hline 22) Benzolc acid & 47.8 & 59.9 & & 36.6 & & 36.6 \\
\hline 23) bls(2-Chloroethoxy) methane & 107.5 & 110.3 & 67.8 & 2.1 & & 2.1 \\
\hline 24) 2,4-Dichlorophenol & 108.8 & 103.9 & 0.4 & 63.7 & & 63.7 \\
\hline 25) 1,2,4-Trichlorobenzene & 102.6 & 99.8 & 59.9 & 1.0 & 0.8 & 1.8 \\
\hline 26) Naphthalene & 104.8 & 99.5 & 60.9 & 2.3 & 42.7 & 44.9 \\
\hline 27) 4-Chloroaniline & 65.1 & 69.2 & 36.0 & 0.1 & & 0.1 \\
\hline 28) Hexachlorobutadiene & 101.7 & 99.1 & 61.5 & 0.8 & 0.1 & $0 . \overline{9}$ \\
\hline 29) 4-Chloro-3-methylphenol & 108.0 & 115.4 & 8.7 & 59.5 & & 59.5 \\
\hline 30) 2-Methylnaphthalene & 103.3 & 85.4 & 55.5 & 2.0 & 47.6 & 49.6 \\
\hline 31) *Acenaphthene-d10 & 100.0 & 100.0 & 100.0 & 100.0 & 100.0 & 100.0 \\
\hline 32) Hexachlorocyclopentadlene & 112.5 & 117.3 & 48.1 & 0.6 & & 0.6 \\
\hline 33) 2,4,6-Trichlorophenol & 112.9 & 103.9 & & 67.9 & & 67.9 \\
\hline 34) 2,4,5-Trichlorophenol & 102.5 & 99.1 & & 65.8 & & 65.8 \\
\hline 35) 2-Chloronaphthalene & 104.0 & 98.8 & 60.4 & 2.3 & $51 . \overline{4}$ & 53.7 \\
\hline 36) \#2-Fluoroblphenyl & 107.2 & 102.6 & 63.5 & 2.5 & 52.8 & 55.3 \\
\hline 37) 2 -Nitroaniline & 113.3 & 117.7 & 75.1 & 2.5 & & 2.5 \\
\hline 38) Dimethylphthalate & 113.2 & 109.9 & 67.7 & 3.4 & & 3.4 \\
\hline 39) Acenaphthylene & 86.6 & 79.6 & 50.6 & 1.9 & 43.2 & $\overline{45.1}$ \\
\hline 40) 2,6-Dinitrotoluene & 109.5 & 110.4 & 66.4 & 2.2 & & 2.2 \\
\hline 41) 3-Nitroaniline & 75.3 & 72.7 & 67.9 & 1.0 & 0.1 & 1.1 \\
\hline
\end{tabular}


TABLE 19. (contd)

\begin{tabular}{|c|c|c|c|c|c|c|}
\hline $\begin{array}{l}\text { Sample: Tank U110 Matrix Spike } \\
\text { No. Compound Name }\end{array}$ & Step 1 & $R_{\theta}-S t \theta 01$ & P2BN & P2A & P3BN & $\begin{array}{r}\text { Total } \\
\text { Recovery }\end{array}$ \\
\hline 42) Acenaphthene & 110.7 & 108.9 & 68.4 & 2,8 & 57.9 & 60.7 \\
\hline 43) 2,4-Dinitrophenol & 24.5 & 35.0 & & 62.1 & & 62.1 \\
\hline 44) 4-Nitrophenol & 108.4 & 113,3 & & 75.1 & & 75.1 \\
\hline 45) Dibenzoturan & 109.1 & 106.0 & $\overline{66.6}$ & 2.9 & 56.9 & 59.9 \\
\hline 46) 2,4-Dinitrotoluen $\theta$ & 112.8 & 108.6 & 68.4 & 2.8 & & 2.8 \\
\hline 47) Diethylphthalate & 114.0 & 114.2 & 71.2 & 3.6 & 0.2 & 3.9 \\
\hline 48) 4-Chlorophenyl-phenylether & 69.2 & 67.6 & 40.3 & 1.9 & 38.4 & 40.2 \\
\hline 49) Fluorene & 110.7 & 108.7 & 68.5 & 2.9 & 60.4 & 63.3 \\
\hline 50) $4-$-Nitroanilline & 91.3 & 80.8 & 56.5 & 1.2 & & 1.2 \\
\hline 51) *Phenanthrene-d10 & 100,0 & 100.0 & 100.0 & 100.0 & 100.0 & 100.0 \\
\hline 52) 4,6-Dinitro-2-methylphenol & 43.0 & 51.9 & & 69.3 & & 69.3 \\
\hline 53) $\mathrm{N}$-Nitrosodlphonylarnino & 120.6 & 113.0 & 67.9 & 2.2 & & 2.2 \\
\hline 54) \#2,4,6-Tribromophenol & 110.4 & 100.3 & & 67.9 & & 67.9 \\
\hline 55) 4-Bromopheny-phenylether & 109.4 & 100.4 & 66.5 & 3.0 & 60.4 & 63.4 \\
\hline 56) Hexachlorobenzene & 112.8 & 99.9 & 69.6 & 3.1 & 0.2 & 3.3 \\
\hline 57) Pentachlorophenol & 109.2 & 98.1 & & 82.9 & & 82.9 \\
\hline 58) Phenanthrene & 111.1 & 104.9 & 71.8 & 3.3 & 63.8 & 67.1 \\
\hline 59) Anthracene & 99.0 & 91.2 & 57.9 & 2.3 & 52.4 & $54 . \overline{7}$ \\
\hline 60) Di-n-butylphthalate & 110.3 & 101.6 & 71.5 & 3.8 & 0.0 & 3.8 \\
\hline 61) Fluoranthene & 107.1 & 96,8 & 69.4 & 3.2 & 63.3 & 66.5 \\
\hline 62) *Chrysen $\theta-d 12$ & 100.0 & 100.0 & 100.0 & 100.0 & 100.0 & 100.0 \\
\hline 63) Pyrene & 101.1 & 108.5 & 69.4 & 2.9 & 67.6 & 70.5 \\
\hline 64) \#Terphenyl-d14 & 93.1 & 103.3 & 66.4 & 2.9 & 65.4 & 68.3 \\
\hline 65$)$ Butylbenzylphthalate & 101.6 & 113.0 & 70.1 & 3.3 & & 3.3 \\
\hline 66) 3,3'-Dichlorobenzidine & 55.0 & 51.0 & 48.3 & & & 0.0 \\
\hline 67) Benzo(a)anthracene & 98.1 & 107.5 & 66.7 & 2.8 & 67.4 & 70.2 \\
\hline 68) Chrysent & 95.7 & 108.4 & $70 . \overline{6}$ & 3.1 & 73.3 & 76.4 \\
\hline 69) BIs(2-Ethylhexyl)phthalate & 94.4 & 101.1 & $63 . \overline{3}$ & 3.2 & 0.3 & 3.5 \\
\hline 70) *Perylene-d12 & 100.0 & 100.0 & 100.0 & 100.0 & 100.0 & 100.0 \\
\hline 71) DI-n-octylphthalate & 110.2 & 95.7 & 63.5 & 3.4 & 0.2 & 3.7 \\
\hline 72) Benzo(b)fluoranthene & 118.3 & 111.0 & 67.9 & 3.1 & 61.0 & 64.2 \\
\hline 73) Benzo(k)fluoranthene & 127.2 & 86.0 & 65.6 & 3.2 & 67.3 & 70.5 \\
\hline 74) Benzo(a)pyrene & 99.3 & 89.0 & 53.5 & 2.1 & 52.1 & 54,2 \\
\hline 75) Indeno $(1,2,3-c 0)$ pyrene & 145.3 & 114.5 & 68.7 & 3.0 & 63.8 & 66.9 \\
\hline 76) Dibenz $(a, h)$ anthracene & 107.5 & 98.3 & 62.1 & 2.6 & 58.1 & 60.8 \\
\hline 77) Benzo $(g, h, 1)$ perylene & 120.3 & 114.3 & 66.6 & 2.8 & 60.9 & 63.7 \\
\hline NPH (Paraffin Hydrocarbon, C12-C & 25.3 & 27.8 & 12.9 & 0.3 & & 0.3 \\
\hline
\end{tabular}

* Compound is an Internal Standard

\# Compound is a Surrogate Splke 
TABLE 20. Percent Recovery Results for Single She1l Tank U110 Matrix Spike Duplicate, Inttial PNL Cleanup Method

\begin{tabular}{|c|c|c|c|c|c|c|}
\hline $\begin{array}{l}\text { Sample: Tank U1 } 10 \text { Matrlx Spike Dupllcate } \\
\text { No. Compound Name }\end{array}$ & Slep 1 & $A_{\theta}-\operatorname{St\theta p} 1$ & P2BN & P2A & P3BN & $\begin{array}{r}\text { Total } \\
\text { Recovery }\end{array}$ \\
\hline 1) ${ }^{1}, 4$-Dlchlorobenzene-d4 & 100.0 & 100.0 & 100.0 & 100.0 & 100.0 & 100,0 \\
\hline 2) \#2-Fluorophenol & 98.8 & 101.9 & 0.3 & 46.9 & 0.2 & 47.1 \\
\hline 3) \#Phenol-d5 & 118.7 & 114,9 & 1.9 & 56.2 & & 56,2 \\
\hline 4) Phenol & 117.4 & 108.5 & 2.2 & 57.6 & & 57.6 \\
\hline 5) bls(2-Chloroethyl)ether & 80.2 & 109.2 & 57,0 & 0.2 & & 0.2 \\
\hline 6) 2-Chlorophenol & 94.3 & 97.5 & 0.6 & 48.1 & & 48,1 \\
\hline 7) 1,3-Dlchlorobenzene & 92.3 & 90.3 & 50.7 & 0.2 & 31.9 & 32.1 \\
\hline 8) 1,4-Dlchlorobenzene & 97.4 & 94.2 & 50.3 & 0.2 & 33.7 & 33,9 \\
\hline 9) Benzylalcohol & 103.2 & 114.7 & 62.9 & 0.8 & & 0.8 \\
\hline 10) 12-Dlchlorobenzene & 90.9 & 91.6 & 51.7 & 0.2 & 34,3 & 34.5 \\
\hline 11) 2-Methylphenol & 80.8 & 85.1 & 9.0 & 39.7 & & 39.7 \\
\hline 12) bls(2-Chlorolsopropyl)ether & $110 . \overline{8}$ & 123.7 & 80.1 & 0.2 & & 0.2 \\
\hline 13) 4-Methylphenol & 86.8 & 91.6 & 7.4 & 44,0 & & 44,0 \\
\hline 14) $N-N \mid$ troso-dl-n-propylamine & 115.2 & 128.4 & 73.3 & 0.3 & & 0.3 \\
\hline 15) Hexachloroethane & 91.9 & 94.1 & 50.8 & 0.1 & 13.1 & 13.2 \\
\hline 16) Naphthalene-d8 & 700.0 & 100.0 & 100.0 & 100.0 & 100.0 & 100,0 \\
\hline 17) \#Nitrobenzene-d5 & 97.1 & 104.2 & 66.0 & 0.2 & 0.0 & 0.2 \\
\hline 18) Nitrobenzene & 133.6 & 145.0 & 91.6 & 0.4 & & 0.4 \\
\hline 19) Isophororie & 68.7 & 108.2 & 64.1 & 0.3 & & 0.3 \\
\hline 20) 2 -Niltrophenol & 108.3 & 109.9 & 0.3 & 65.8 & & 65.8 \\
\hline 21) 2,4-Dimethylphenol & 8.2 & 9.1 & 4.3 & 3.0 & & 3.0 \\
\hline 22) Benzolc acld & 37.5 & 40.5 & & 15.6 & & 15,6 \\
\hline 23) bls $(2$-Chloroethoxy)metriane & 104.9 & 107.7 & 70.7 & 0.3 & & 0.3 \\
\hline 24) 2,4-Dlchlorophenol & 103.8 & 103.4 & 0.6 & 65.5 & & 65.5 \\
\hline 25) 1,2,4-Trlchlorobenzene & 97.6 & 97.5 & 59.7 & & 49.3 & 49.3 \\
\hline 26) Naphthalene & 97.4 & 99.2 & 60.6 & 3.2 & 52.0 & 56.2 \\
\hline 27) 4-Chloroanilline & 41.9 & & 1.0 & 0.1 & & 0.1 \\
\hline 28) Hexachlorobutadiene & 95.7 & 95.4 & 62.0 & 0.2 & 18.6 & 18.8 \\
\hline 29) 4-Chloro-3-methylphenol & 103.7 & 115.0 & 7.6 & 62.2 & & 62.2 \\
\hline 30) 2-Methylnaphthalene & 97.3 & 91.5 & 61.2 & 0.3 & 58.6 & 589 \\
\hline 31) "Acenaphthene-d10 & 100.0 & 100.0 & 100.0 & 100.0 & 100.0 & 100.0 \\
\hline 32$)$ Hexachlorocyclopentadiene & 117.7 & 118.8 & 55.9 & 0.1 & 16.7 & 16.7 \\
\hline 33) 2,4,6-Trichlorophenol & 111.8 & 100.6 & & 66.7 & & 66.7 \\
\hline 34) 2,4,5-Trlchlorophenol & 104.3 & 94.9 & & 66.4 & & 66.4 \\
\hline 35) 2-Chloronaphthalene & 101.0 & 96.4 & 62.5 & 0.3 & 61.1 & 61.4 \\
\hline 36) \$2-Fluoroblphenyl & 106.7 & 98.2 & 62,6 & 0.3 & 61.9 & 62.2 \\
\hline 37) $2-$ Nitroanilline & 96.9 & 98.6 & 69.4 & 0.3 & & 0.3 \\
\hline 38) Dimethylphthalate & 115.7 & 109.4 & 72.6 & 0.5 & & 0.5 \\
\hline 39) Acenaphthylene & 70.9 & 65.0 & 42.5 & 0.2 & 41.8 & 42.0 \\
\hline 40) 2,6-Dinitrotoluene & 110.2 & 109.0 & 71.8 & 0.2 & & 0.2 \\
\hline 41) 3 -Nitroanlline & 3.6 & & 5.9 & & & 0.0 \\
\hline
\end{tabular}


TABLF 20. (contd)

\begin{tabular}{|c|c|c|c|c|c|c|}
\hline $\begin{array}{l}\text { Sample: Tank L1 } 10 \text { Matrlx Splko Duplloate } \\
\text { No. Compound Name }\end{array}$ & Step 1 & $B \theta-S t e p 1$ & PRBN & $P 2 A$ & PABN & $\begin{array}{r}\text { Total } \\
\text { Aecovery }\end{array}$ \\
\hline 42) Acenaphtheno & 110.2 & 105,3 & 69.2 & 0.3 & 68.6 & 66,9 \\
\hline 43) 2,4-Dinltrophienol & 42.8 & 46.4 & & 59.2 & & 69,2 \\
\hline 44) 4-Nitrophenol & 131.4 & 133.8 & & 91.9 & & 91,9 \\
\hline 45 Dibenzofuran & 109.4 & 103.7 & 70.2 & 0.4 & 67,3 & 67.7 \\
\hline 46) 2,4-Dinltrotoluene & 119,2 & 109,4 & 74.8 & 0.4 & & 0.4 \\
\hline 47) Dlothylphthalato & Tीव.9 & 107.8 & 74.3 & 0.6 & 0.3 & 0.8 \\
\hline 48$)$ 4-Dhlorophenyl-pitienylether & 69.5 & 66,6 & $4 \sqrt{15}$ & 0.2 & 45.9 & 46.1 \\
\hline 49) Fluorene & 110.6 & 104,9 & 71.4 & 0.4 & 70.5 & 70.9 \\
\hline 50) $4-N|\operatorname{troan}| l|r| \theta$ & 29.9 & 30.3 & 36.1 & & & 0.0 \\
\hline 51) *hananthrene-d10 & 100,0 & 100.0 & 100.0 & 100.0 & 100,0 & 100,0 \\
\hline 52) $4,6-0$ nitro-2-methylphenol & 62.4 & 66,4 & & 73.6 & & 733.6 \\
\hline 53) $N$-Nitrosodiptienylamine & 01.6 & 56.3 & 45.9 & 0.2 & & 0.2 \\
\hline 54 \#2,4,6-..Trlbromophenol & 117.4 & 105,1 & & 72.9 & & 72.9 \\
\hline 55 4-Bromopheny -phenylether & 108.3 & 102.9 & 70.6 & 0.5 & 71.4 & 71.8 \\
\hline 56) Hexachlorobenzene & 112.0 & 99.6 & 74.8 & 0.6 & 42.2 & 42.7 \\
\hline 57) Pentachlorophenol & 119.7 & $1 1 \longdiv { 6 }$ & & 93,4 & & 93.4 \\
\hline 58) Phonanthrene & 108.2 & 102.6 & 75.0 & 0.5 & 74.4 & 749 \\
\hline 59) Arithracent & 51.4 & 49.6 & 33,1 & 0.5 & 329 & 33,4 \\
\hline 60) DI-n-butylonthalate & 106.7 & 103,3 & 75.3 & 0.7 & 0.0 & 0.7 \\
\hline 61) Fluorantheng & 104.8 & 96.3 & 71.9 & 0.5 & 72.7 & 73.2 \\
\hline 62) "Chrysene-d12 & 100.0 & 100,0 & 100.0 & 100.0 & 100,0 & 100.0 \\
\hline 63) Pyreno & 95.8 & 101.6 & 66.0 & 0.5 & 72.4 & 72.9 \\
\hline 64) \#Terphonyl-d14 & 90.0 & 93,8 & 62.0 & 0.6 & 69.4 & 70,0 \\
\hline 65) Butylbenzylphthalate & 99.5 & 105.5 & 68.4 & 0.6 & & 0.6 \\
\hline 66) 3,3'-Dlchlorobenzldine & & & & & & 0.0 \\
\hline 67) Benzo(a)anthracene & 84.8 & 93.0 & 58.1 & 0.5 & 63.8 & 64.3 \\
\hline 68) Chrysene & 99.2 & 102,8 & 68.1 & 0.6 & 76.8 & 77.4 \\
\hline 69) Bis(2-Ethylhexyl)phthalate & 94.5 & 97.1 & 62.0 & 0.7 & 0.1 & 0.9 \\
\hline 70) \#Perylene-d12 & 100.0 & 100.0 & 100.0 & 100.0 & 100.0 & 100.0 \\
\hline 71) DI-n-ootylphthalate & 113.8 & 100.2 & 71.8 & 0.6 & 0.1 & 0.7 \\
\hline 72) Benzo(b)fluoranthene & 119.0 & $106 . \overline{4}$ & 79.0 & 0.5 & 71.2 & 74.7 \\
\hline 73) Benzo (k) fluoranthene & 113.9 & 109.0 & 66.0 & 0.5 & 74.8 & 75.2 \\
\hline 74) Benzo(a)pyrana & 35.7 & 34.6 & 22.6 & 0.1 & 22,4 & 22.5 \\
\hline 75) Indeno $(1,2,3-0$-d)pyreno & 87.7 & 116.1 & 74.2 & 0.5 & 71.6 & 72.1 \\
\hline 76) Dibenz (a,h)anthracene & 105.1 & 102.9 & 65.9 & 0.4 & 65.4 & 65.8 \\
\hline 77) Benzo(g, $h$, l) perylene & 116.6 & 1129 & 73.7 & 0.5 & 70.2 & 70.6 \\
\hline NPH (Parafflin Hydrocarbon, 012-C14) & 20.7 & 23.6 & 11.0 & & 0.8 & 0.8 \\
\hline
\end{tabular}

* Compound ls an Internal Standard

\# Compound is a Surrogate Splke 
TABLE_. Results for Single Shell Tank Bl10 Using Inttial PNL. Cleanup Method

Sample: Tank 1110

No. Compolund Name

1) 1,4-Dlohiolobenzene-d4

\#2-Fluoroptiano

3) \#Prenol-dE

4) Prieno

6) ble(e-Chloroothyl)etrier

6) 2-Chorophenol

3) 13-Dlchlorobenzeria

B) 1,4-Dluhlorobenzene

9) Benzyl alcotio

10) 1,2-Dlohlorobenzena

11) 2-Mettylpheno

12) blo(2-Chlorolsopropyl)ether

13) 4-Methylphenol

14) $N-N[$ troso-0] $\cdots n$-propylamino

15) Hexachloroethane

16) Naphthalene-d8

17) \#itrobenzene-db

18) Nitrobenzene

$19)$ sophorone

20) 2-Nitiophonol

21) 2,4-D methylphenol

22 Benzolo acld

23 blet2-Chloroetnoxy) methane

24 2,4-Dlchlorophenol

25) 1,2,4-Trlchlorobenzene

26) Naphifialene

27) 4-Chloroanil|n日

28) Hexachlorobutadiene

29) 4-Chloro-3-methylphenol

30) 2-Methylnaphthalene

31) *Acenaphthen $\theta-$ d 10

32 Hexachlorocyolapentadlene

33) 2,4,6-Trictilorophenol

34 2,4,5-Trich loropherio

35 2-Chloronaphthaleno

36) \#2-Fluoroblpheny

37) 2-Nitroanillne

38) Dlmethylphthalate

39) Acenaphithylene

40) $2,6-$ - Inltrotoluene

41) 3-Nitroanillire

\begin{tabular}{|c|c|c|c|c|c|}
\hline Step 1 & E2N & E2A & $E 2 B$ & $E B N$ & $\begin{array}{l}\text { Total } \\
\text { Mecovery }\end{array}$ \\
\hline 100.0 & 100.0 & 100,0 & 100.0 & 1000 & 100,0 \\
\hline 98,1 & 0.2 & 49.7 & & & 49.7 \\
\hline \multirow[t]{12}{*}{104.2} & 1,8 & 55.1 & & & 66.1 \\
\hline & & & & & \\
\hline & & & & & \\
\hline & & & & & \\
\hline & & & & & \\
\hline & & & & & \\
\hline & & & & & \\
\hline & & & & & \\
\hline & & & & & \\
\hline & & & & & \\
\hline & & & & & \\
\hline & & & & & \\
\hline \multirow{16}{*}{$\frac{100.0}{02.6}$} & & & & & \\
\hline & 100.0 & 1000 & 100.0 & 100.0 & 100.0 \\
\hline & 50.1 & 0.4 & 0.3 & & 0.6 \\
\hline & & & & & \\
\hline & & & & & \\
\hline & & & & & \\
\hline & & & & & \\
\hline & & & & & \\
\hline & & & & & \\
\hline & & & & & \\
\hline & & & & & \\
\hline & & & & & \\
\hline & & & & & \\
\hline & & & & & \\
\hline & & & & & \\
\hline & & & & & \\
\hline \multirow[t]{5}{*}{100,0} & 100,0 & 100.0 & 100.0 & 1000 & 100.0 \\
\hline & & & & & \\
\hline & & & & & \\
\hline & & & & & \\
\hline & & & & & \\
\hline 95.4 & 55.6 & 0.7 & 1.4 & 19,8 & 21.9 \\
\hline & - & & & & \\
\hline & & & & & \\
\hline & & & & & \\
\hline & & & & & \\
\hline
\end{tabular}


IABLEL21. (contd)

\begin{tabular}{|c|c|c|c|c|c|c|}
\hline \multirow{2}{*}{\multicolumn{7}{|c|}{$\begin{array}{l}\text { Sampla: Tank B110 } \\
\text { No, Cornpound Name } \\
\text { 42) Aoenaphiheno }\end{array}$}} \\
\hline & & & & & & \\
\hline 43 2,4-Dinitrophend & & & & & & \\
\hline 44) 4 -Nilroptienol & & & & & & \\
\hline 46$)$ Dbenzofuran & & & & & & \\
\hline 46$) 2,4$-Dinitrotoluene & & & & & & \\
\hline 47 Dletrylphthalate & & & & & & \\
\hline 48 4-Chorapieny-prienylether & & & & & & \\
\hline 49$)$ Fluorene & & & & & & \\
\hline 50) 4-Nitroanlline & & & & & & \\
\hline S1) Ephenanthreno-d10 & 100,0 & 100.0 & 100.0 & 100,0 & 100.0 & 100.0 \\
\hline 32$) 4,0-0$ Intro-e-methylphenol & & & & & & \\
\hline 63) $N$-Nitrosodiphenylamine & & & & & & \\
\hline 64) \#2,4,6-Tribromophenol & 110.8 & & 69.8 & & & 69.8 \\
\hline 65) 4-Bromophenyl-phenylether & & & & & & \\
\hline 56) Hexachlorobenzene & & & & & & \\
\hline 57 Pentaohlorophenol & & & & & & \\
\hline 58 Phemanthrera & & & & & & \\
\hline 50) Anthrageno & & & & & & \\
\hline 60) DI-n-butylphthalate & & & & & & \\
\hline 61) Fluoranthene & & & & & & \\
\hline 62 Chrysene-c12 & 100.0 & 100,0 & 100,0 & 100.0 & 100.0 & 100.0 \\
\hline 63) Pyrene & & & & & & \\
\hline 64) \#-Terpheny-d14 & 86.9 & 66.6 & 1,7 & 4.2 & 30.2 & 42.1 \\
\hline 65) Butylbenzylphthalate & & & & & & \\
\hline 66) 3,3-Dlohlorobenzldine & & & & & & \\
\hline 67) Banzo(a)anthraceno & & & & & & \\
\hline 68) Chlysent & & & & & & \\
\hline 69) Bls $(2-E$ thy hexyl)phithalate & & 2,1 & & & & \\
\hline 70) tperylene-di2 & 100.0 & 100.0 & 100.0 & 100.0 & 100.0 & 100.0 \\
\hline 77 DT-n-ootylphthalato & & & & & & \\
\hline 72 Benzo(b)fluoranthene & & & & & & \\
\hline 73 Benzo(k)fluoranthene & & & & & & \\
\hline 74 Benzo(a)pyrene & & & & & & \\
\hline 75 Indeno $(1,2,3-c$ d)pypene & & & & & & \\
\hline 76 Dlbenz a,h)anthracene & & & & & & \\
\hline 77) Benzo $(g, h, i)$ perylene & & & & & & \\
\hline NPH (ParaftIn Hydrocarbon, $\mathrm{C12}-\mathrm{C} 12$ & 170,8 & 85.9 & 1.5 & 4,4 & & 8.9 \\
\hline
\end{tabular}

* Compound ls ari Internal Standard

* Compound lo a Surrogate Splke 
IABLEZ22. Percent Recovery Results for Single Shell Tank B110 Matrix Sptke, EPA 3630 Cleanup Method

\begin{tabular}{|c|c|c|c|c|c|c|}
\hline \multirow[t]{2}{*}{$\begin{array}{l}\text { Sample: Tank B } 10 \text { Matrlx Splke } \\
\text { No. Compound Narne }\end{array}$} & Step 1 & E2N & E2A & $E 2 E$ & E3N & $\begin{array}{r}\text { Total } \\
\text { Recovery }\end{array}$ \\
\hline & 100.0 & 100.0 & 100,0 & 100.0 & 100.0 & 100.0 \\
\hline 2) \#2-Fluorophenol & 99.0 & 3.6 & 70.4 & & 0,0 & 70,4 \\
\hline 3) \#phend-d5 & 109,9 & 2.1 & 76.0 & 0.1 & & 75,1 \\
\hline 4) Phanol & 105,0 & 23 & 64.8 & & & 64,8 \\
\hline 5) bla(2-chloroethyl)ether & 103,6 & 64,8 & 0.4 & 50.5 & & 50.8 \\
\hline 6) 2-Chlorophenol & 94,8 & 0,4 & 68.9 & & & 68.9 \\
\hline 3) 1,3-0lohlorobenzent & 93.8 & 51.8 & 0.3 & 2,6 & 0.5 & 3,3 \\
\hline 8) 1,4-Dlofilorobenzeno & 98.4 & 54.1 & 0,3 & 2.5 & 0.9 & 3.7 \\
\hline 3) Benzy alooho & 97.5 & 58.1 & 7.7 & 5.5 & & 7,2 \\
\hline 10) 1,2-Dlchlorobenzone & 93.0 & 52.5 & 0.3 & 2.7 & 4,4 & 7,4 \\
\hline 11) 2-Mothylphend & 96.8 & 11,8 & 64.6 & 0.7 & & 65.3 \\
\hline 12) bls(2-Chlorolsopropyl)other & 106,8 & 68.2 & & 3.3 & 0,1 & 3,4 \\
\hline 13) 4-Mettiylphenol & 97.2 & 9,2 & 60.8 & 0.5 & & 61.3 \\
\hline (14) $N-N i t r o s o-d-n-p r o p y l a m i n \theta$ & 96.8 & 63,0 & 0.6 & 2.9 & & 3.5 \\
\hline 15) Hexaohlorogthane & 93,3 & 55.1 & 0.2 & 2.6 & & 2.7 \\
\hline 16$)$ Naphthalane-de & 100,0 & 100,0 & 100,0 & 100.0 & 100.0 & 100.0 \\
\hline 17) \#Ntroberzen $\theta-$ dS & 99.9 & 67.1 & 0.4 & 3.2 & 12.2 & 15.7 \\
\hline 18) Nitrobenzene & 102.3 & 62,3 & 0,4 & 3.2 & 13.6 & 17.2 \\
\hline 19) lsophorone & 98.7 & 60.4 & 0.9 & 3.5 & & 4.4 \\
\hline 20) 2-Nitrophenol & 98.1 & 0.2 & 74.9 & & & 74.9 \\
\hline 21 2,4-Dimethylphenol & 91.5 & 32.2 & 18.8 & 2.0 & 0.3 & 21.1 \\
\hline 22) Benzolo aold & 71,9 & & 64.7 & & & 64.7 \\
\hline 23) bls(2-Chloroethoxy)methano & 109.3 & 66.6 & 0.7 & 4.0 & & 4.7 \\
\hline 24) 2,4-Elohlorophenol & 110.0 & 0.5 & 77.3 & & & 77.3 \\
\hline 25) 1, 2, - Trlohlorobenzene & 99.5 & 59.7 & 0.5 & 3,6 & 0,6 & 4.7 \\
\hline 26) Naphithalene & 106.5 & 63,4 & $\overline{1.1}$ & 4.0 & 51.7 & 56.8 \\
\hline 27) 4-Chloroanilline & 114,7 & & & 51.2 & & 51.2 \\
\hline 28) Hexachlorobutadieno & 106,2 & 61,3 & 0.5 & 3,8 & & 4.3 \\
\hline 29) 4-Chlaro-3-methylphenol & 107.2 & 9,6 & 70.8 & 0.4 & & 71.2 \\
\hline 30) 2-Methylnaphthalene & 90.1 & 62.7 & 0.7 & 4,1 & 54.2 & 59.0 \\
\hline 31) *Aoernaphthene-d10 & 100.0 & 100.0 & 100.0 & 100.0 & 100.0 & 100,0 \\
\hline 32) Hexachlorocyolopentadiene & 80.5 & 48.4 & & 1.2 & & $\sqrt{1.2}$ \\
\hline 33) 2,4,6-Trlahlorophenol & 101.8 & & 83.0 & & & 83.0 \\
\hline 34) $2,4,5$-Trlकhorophend & 91.5 & & 75.7 & & & 75.7 \\
\hline 35) 2-Chloronafinthalone & 99.2 & 62.9 & 0.8 & 3.9 & 48.7 & 53.4 \\
\hline 36) \#2-Fluoroblphenyl & 103.2 & 64.9 & 0.9 & 4.2 & 54.7 & 59.9 \\
\hline 37) $2-N \mid \operatorname{troanillin} \theta$ & 95.5 & 54.0 & 1.3 & 3.2 & & 4.5 \\
\hline 38) Dimethylphthalate & 102.2 & 68.3 & 1.5 & 4.2 & & 5.7 \\
\hline 39) Aoeriaphthylene & 96,2 & 62.6 & & 3.8 & 52,3 & 56.2 \\
\hline 40) 2,6-Dinitrotoluene & 87.4 & 67.6 & 0.9 & 2.7 & & $3 . \overline{6}$ \\
\hline 41) 3 -Nltroanillne & 61,8 & 20.4 & & 16.3 & & 16,3 \\
\hline
\end{tabular}




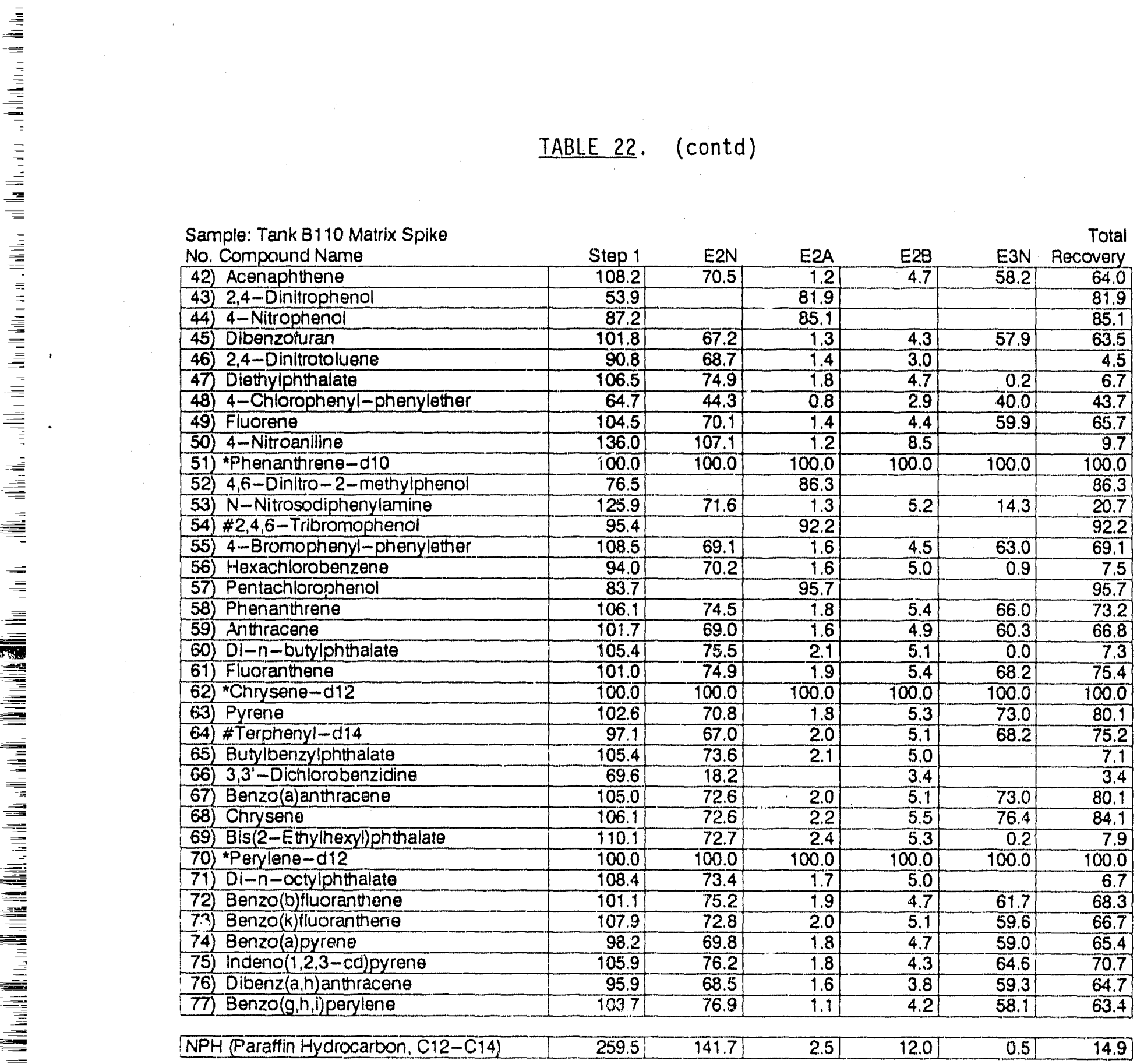

* Compourid is an intemal Standard

\# Compound is a Surrogate Spike 
IABLE 23. Percent Recovery Results for Single Shell Tank B110 Matrix Spike Duplicate, EPA 3630 Cleanup Method

\begin{tabular}{|c|c|c|c|c|c|c|}
\hline $\begin{array}{l}\text { Sarnple: Tank } 8110 \text { Matrix Spike Duplicate } \\
\text { No. Compound Name }\end{array}$ & Step 1 & E2N & E2A & $\mathrm{E} 2 \mathrm{~B}$ & E3N & $\begin{array}{r}\text { Total } \\
\text { Recovery }\end{array}$ \\
\hline 1) ${ }^{1,4}$-Dichlorobenzene-d4 & 100.0 & 100.0 & 100.0 & 100.0 & 100.0 & 100.0 \\
\hline 2) \#2-Fluorophenol & 102.9 & 0.2 & 63.9 & & 0.0 & 64.0 \\
\hline 3) \#Phenol-d5 & 118.7 & 2.4 & 69.8 & & & 69.8 \\
\hline 4) Phenol & 110.0 & 2.6 & 62.0 & & & 62.0 \\
\hline 5) bls(2-Chloroethyl)ether & 110.8 & 51.7 & 1.0 & 44.6 & & 45.6 \\
\hline 6) 2-Chlorophenol & 103.3 & 0.6 & 66.2 & & & 66.2 \\
\hline 7) 1,3-Dichlorobenzene & 99.7 & 48.4 & 0.8 & 0.3 & 3.7 & 4.8 \\
\hline 8) 1,4-Dichlorobenzene & 102.2 & 49.3 & 0.8 & 0.3 & 5.0 & 6.2 \\
\hline 9) Benzyl aicohol & 103.4 & 53.5 & 2.9 & 1.4 & & 4.2 \\
\hline 10) 1,2-Dichlorobenzene & 102.6 & 49.3 & 0.8 & 0.3 & 8.5 & 9.6 \\
\hline 11) 2-Methylphenol & 100.2 & 13.7 & 50.9 & & & 50.9 \\
\hline 12) bls(2-Chlorolsopropyl)ether & 115.5 & 63.7 & 1.1 & 0.5 & 0.2 & 1.8 \\
\hline 13) 4-Methylphenol & 106.3 & 10.8 & 56.4 & & & 56.4 \\
\hline 14) $N$-Nitroso-di-n-propylamine & 100.2 & 58.9 & 1.5 & 0.4 & & 1.9 \\
\hline 15) Hexachloroethane & 97.5 & 50.2 & 0.7 & 0.3 & 0.5 & $\overline{1.5}$ \\
\hline 16) *Naphthalene-d8 & 100.0 & 100.0 & 100.0 & 100.0 & 100.0 & 100.0 \\
\hline 17) \#Nitrobenzen $\theta-d 5$ & 102.6 & 62.3 & 0.9 & 0.3 & 12.2 & 13.4 \\
\hline 18) Nitrobenzene & 104.6 & 61.7 & 1.1 & 0.3 & 13.9 & 15.3 \\
\hline 19) Isophorone & 103.4 & 57.3 & 2.1 & 0.6 & & 2.7 \\
\hline 20) 2-Nitrophenol & 101.8 & 0.4 & 71.8 & & & 71.8 \\
\hline 21) 2,4-Dimethylphenol & 92.2 & 34.6 & 17.8 & 0.3 & 0.3 & $\overline{18.4}$ \\
\hline 22) Benzoic acid & 74.2 & & 53.5 & & & 53.5 \\
\hline 23) bis(2-Chloroethoxy)methane & 113.4 & 64.0 & 1.8 & 0.7 & & 2.5 \\
\hline 24) 2,4-Dichlorophenol & 108.9 & 0.6 & 72.9 & & & 72.9 \\
\hline 25) 1,2,4-Trichlorobenzene & 106.9 & 59.0 & 1.2 & 0.7 & 5.1 & 6.9 \\
\hline 26) Naphthalene & 110.3 & 61.8 & 1.4 & 0.7 & 41.2 & 43.3 \\
\hline 27) 4-Chloroaniline & 123.1 & & & 47.6 & & 47.6 \\
\hline 28) Hexachlorobutadiene & 106.6 & 56.8 & 1.1 & 0.8 & 0.7 & 2.6 \\
\hline 29) 4-Chloro-3-methylphenol & 111.2 & 11.4 & 66.0 & & & 66.0 \\
\hline 30) 2-Methylnaphthalene & 94.6 & 61.3 & 1.8 & 1.0 & 45.3 & 48.1 \\
\hline 31) *Acenaphthene-d10 & 100.0 & 100.0 & 100.0 & 100.0 & 100.0 & 100.0 \\
\hline 32) Hexachlorocyclopentadiene & 84.7 & 45.1 & 0.6 & 0.2 & & 0.8 \\
\hline 33) 2,4,6-Trichlorophenol & 106.4 & & 78.1 & & & 78.1 \\
\hline 34) 2,4,5-Trichlorophenol & 94.4 & & 71.6 & & & 71.6 \\
\hline 35) 2-Chloronaphthalene & 103.8 & 60.6 & $1 . \overline{9}$ & 1.2 & 43.2 & 46.3 \\
\hline 36) \#2-Fluoroblohenyl & 105.4 & 61.4 & 2.1 & 1.3 & 46.9 & 50.2 \\
\hline 37) 2-Nitroaniline & 99.7 & 57.4 & 2.8 & 0.8 & & 3.6 \\
\hline 38) Dimethylphttialate & 104.8 & 63.9 & 3.3 & 1.6 & & 4.9 \\
\hline 39) Acenaphthylene & 98.5 & 59.2 & 2.0 & 1.4 & 44.9 & 48.3 \\
\hline 40) 2,6-Dinitrotoluene & 90.5 & 63.6 & 2.3 & 0.8 & & 3.1 \\
\hline 41) 3-Nitroaniline & 64.7 & 22.2 & 0.5 & 13.4 & & 14.0 \\
\hline
\end{tabular}


TABLE 23. (contd)

Sample: Tank B110 Matrlx Splke Duplicate

No. Compound Name

Step

42) Acenaphthene

43) 2,4-Dinitrophenol

44) 4-Nitrophenol

45) Dibenzofuran

46) 2,4-Dinitrotoluene

47) Diethylph thalate

48) 4-Chlorophenyl-phenylether

49) Fluorene

50) 4-Nitroanilline

51) *Phenanthren $\theta-d 10$

52) 4,6-Dinitro-2-methylpheno

53) $\mathrm{N}-\mathrm{N}$ ltrosodiphenylamine

54) \#2,4,6-Tribromophenol

55) 4-Brornophenyl-phenylether

56) Hexachlorobenzene

57) Pentachlorophenol

58) Phenanthrene

59) Anthracene

60) $D I-n$-butylphthalate

61) Fluoranthene

62) *Chryseno-d12

63) Pyrene

64) \#Terphenyl-d14

65) Butylbenzylphtnalate

66) 3,3'-Dichlorobenzidine

67) Benzo fadanthracene

68) Chrysenta

69) Bis(2-Ethylhexyl)phthalate

70 *Perylen $\theta-d 12$

71) Di-n-octylphthalate

72) Benzo(b)fluoranthene

73) Benzo (k)fluoranthene

74) Benzo (a)pyrene

$75)$ Indeno $(1,2,3$-cd)pyren $\theta$

76) Dihenz $(a, h)$ anthracen $\theta$

\begin{tabular}{|l|l}
\hline 77$)$ Eerso (g,h,iperylene & 109
\end{tabular}

111.6

E2N

E2A

E2B E3N Recovery

Total

$\frac{54.1}{89.7}$

89.7
106.5

63.7

$91.0 \quad 65$

$107.7 \quad 68.6$

$68.3-41.5$

$107.9-65.9$

140.8

100.0
76.4

76.4

100.0

102.2

104.8

99.2

88.7

109.7

103.0

107.8

100.0

103.6

97.8

105.6

70.5

106.8

113.3

110.7

100.0

107.9

105.4

101.0

106.6

100.0

09.1

69.0

69.0

65.0

65.5

69.0

69.0

70.5

100.0

72.5

73.2

22.4

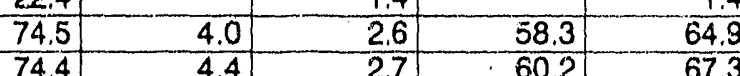

NPH (Paraffin Hydrocarbon, C12-C14)

232.1

72.8

100.0

100.0

5.1

70.8

\begin{tabular}{r|r}
70.8 & 3.7
\end{tabular}

\begin{tabular}{r|r}
64.2 & 4.3 \\
\hline 67.8 & -3.7
\end{tabular}

\begin{tabular}{r|r}
67.8 & 3.7 \\
70.7 & 3.8
\end{tabular}

70.7

64.0

70.7

$\begin{array}{r}3.8 \\ 3.8\end{array}-2.2$

$3.4-1.6$

3.8

\begin{tabular}{r|r}
\hline 51.6 & 56.2 \\
\hline & 86.7
\end{tabular}

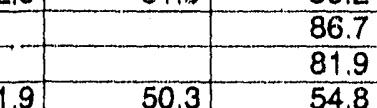

* Compound is an Internal Standard

\# Compound is a Surragate Spike 
TABLE 24. Comparison of the Effect of NPH in Blank Spikes on Cleanup Method Recoveries Including Methylene Chloride Elution

Sample: Blank Splke

No. Compound Name

1) *1,4-Dichlorobenzene-d4

\#2-Fluoropheno

3) \#Phenol-d5

4) Phenol

5) bis(2-Chioroethyl)etter

6) 2-Chlorophenol

7) 1,3-Dir hiorobenzene

8) 1,4-Dichlorobenzene

9) Benzyl alcohol

10) 1,2-Dichlorobenzene

11) 2-Methylphenol

12) bis(2-Chloroisopropyl)ether

13) 4-Methylphenol

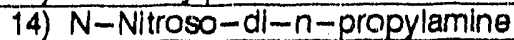

15) Hexachloroethane

16) *Naphthalen $\theta-\mathrm{d} 8$

17) \#Nitrobenzene-d5

18) Nitrobenzene

19) Isophorone

20) 2-Niti'cphenol

21) 2,4-Dimethylphenol

22) Benzoic acid

23) bis(2-Chloroethoxy)methane

24) 2,4-Dichlorophenol

25) 1,2,4-Trichlorobenzene

26) Naphthalene

27) 4-Chloroaniline

28) Hexachlorobutadiene

29) 4-Chloro-3-methylpheno

30) 2--Methylnaphthalene

31) *Acenaphthen $\theta-d 10$

32) Hexachlorocyclopentadiene

33) 2,4,6-Trichlorophenol

34) 2,4,5-Trichlorophenol

35) 2-Chloronaphthalene

36) \#2-Fluorobiphenyl

37) 2-Nitroaniline

38) Dimethylphthalate

39) Acenaphthylene

40) 2,6-Dinitrotoluene

41) 3-Nitroaniline

\begin{tabular}{|c|c|c|c|c|c|}
\hline \multicolumn{3}{|c|}{ PNL with P3BN2 step } & \multicolumn{3}{|c|}{ EPA with E3N2 step } \\
\hline $\mathrm{MS}+\mathrm{NPH}$ & MS only & NPHEffect & $\mathrm{MS}+\mathrm{NPH}$ & MS only & NPHEffect \\
\hline 100.0 & 100.0 & 0.0 & 100.0 & 100.0 & 0.0 \\
\hline 63.1 & 55.9 & 7.2 & 59.7 & 75.7 & -16.0 \\
\hline 66.1 & 58.7 & 7.4 & 61.9 & 73.8 & -11.9 \\
\hline 59.4 & 54.4 & 5.0 & 56.0 & 69.1 & -13.1 \\
\hline 50.4 & 38.2 & 12.2 & 32.6 & 9.6 & 23.0 \\
\hline 65.5 & 57.2 & 8.2 & 58.6 & 70.6 & -12.0 \\
\hline 45.7 & 34.4 & 11.3 & 1.7 & 4.9 & -3.1 \\
\hline 47.4 & 38.1 & 9.3 & 2.0 & 4.8 & -2.8 \\
\hline 3.7 & 1.7 & 2.0 & 5.3 & 9.6 & -4.3 \\
\hline 47.0 & 38.0 & 9.0 & 3.0 & 6.1 & -3.0 \\
\hline 58.0 & 55.3 & 2.7 & 50.3 & 54.6 & -4.3 \\
\hline 73.5 & 58.5 & 15.0 & 16.2 & 19.6 & -3.4 \\
\hline 57.2 & 48.6 & 8.5 & 53.2 & 58.7 & -5.5 \\
\hline 2.3 & 0.9 & 1.4 & 1.6 & 5.2 & -3.6 \\
\hline 42.9 & 15.7 & 27.2 & 1.2 & 4.2 & -3.1 \\
\hline 100.0 & 100.0 & 0.0 & 100.0 & 100.0 & 0.0 \\
\hline 72.3 & 57.8 & 74.5 & 58.5 & 19.5 & 39.0 \\
\hline 74.9 & 60.4 & 14.5 & 63.5 & 22.1 & 41.4 \\
\hline 3.5 & 1.5 & 2.0 & 3.9 & 6.3 & -2.4 \\
\hline 78.2 & 67.9 & 10.4 & 64.6 & 84.1 & -19.5 \\
\hline 36.4 & 38.7 & -2.3 & 30.7 & 24.7 & 6.0 \\
\hline 63.6 & 52.7 & 10.9 & 44.1 & 78.9 & -34.8 \\
\hline 79.9 & 66.5 & 13.3 & 3.8 & 7.1 & -3.3 \\
\hline 74.9 & 70.9 & 4.1 & 68.0 & 82.3 & -14.2 \\
\hline 64.9 & 49.2 & 15.7 & 3.6 & 6.8 & -3.2 \\
\hline 66.3 & 55.3 & 11.0 & 35.9 & 43.8 & -7.9 \\
\hline 2.5 & 1.8 & 0.7 & 78.9 & 87.6 & -8.6 \\
\hline 56.2 & 20.6 & 35.6 & 2.9 & 6.2 & -3.3 \\
\hline 70.1 & 61.9 & 8.2 & 64.4 & 68.7 & -4.2 \\
\hline 70.1 & 61.5 & 8.6 & 41.5 & 47.7 & -6.2 \\
\hline 100.0 & 100.0 & 0.0 & 100.0 & 100.0 & 0.0 \\
\hline 41.8 & 14.8 & 27.1 & 1.0 & 1.8 & -0.8 \\
\hline 83.9 & 71.7 & 12.2 & 67.4 & 81.3 & -13.9 \\
\hline 81.6 & 68.7 & 12.9 & 62.4 & 74.8 & -12.4 \\
\hline 75.8 & 63.0 & 12.7 & 28.8 & 37.4 & -8.6 \\
\hline 80.0 & 64.6 & 15.4 & 45.4 & 51.3 & -5.9 \\
\hline 85.1 & 67.5 & 17.6 & 4.9 & 6.6 & -1.6 \\
\hline 5.4 & 2.8 & 2.5 & 6.9 & 7.9 & -1.1 \\
\hline 78.1 & 64.4 & 13.6 & 47.5 & 52.1 & -4.6 \\
\hline 89.3 & 70.6 & 18.7 & 4.6 & 20.8 & -16.3 \\
\hline 8.1 & 3.8 & 4.3 & 72.4 & 91.9 & -19.5 \\
\hline
\end{tabular}


TABLE 24. (contd)

\begin{tabular}{|c|c|c|c|c|c|c|}
\hline \multirow{2}{*}{$\begin{array}{l}\text { Sample: Blank Spike } \\
\text { No. Compound Name }\end{array}$} & \multicolumn{3}{|c|}{ PNL with P3BN2 step } & \multicolumn{3}{|c|}{ EPA with E3N2 step } \\
\hline & $\mathrm{MS}+\mathrm{NPH}$ & MS only & NPHEffoct & $\mathrm{MS}+\mathrm{NPH}$ & MS only & NPHEffeCt \\
\hline 42) Acenaphthene & 86.7 & 71.6 & 15.1 & 53.8 & 57.4 & $-3 . \overline{6}$ \\
\hline 43) 2,4-Dinitrophenol & 84.8 & 78.9 & 6.0 & 60.1 & 96.6 & -36.5 \\
\hline 44) 4-Nitrophenol & 82.0 & 75.8 & 6.2 & 65.1 & 85,5 & -20.5 \\
\hline 45) Dibenzofuran & 86.1 & 69.7 & 16.4 & 55.5 & 58.6 & -3.1 \\
\hline 46) 2,4-Dinitrotoluene & 87.1 & 69.5 & 17.6 & 5.1 & 16.2 & -11.1 \\
\hline 47) Dlethylphthalate & 5.9 & 3.5 & 2.4 & 6.9 & 8.2 & -1.2 \\
\hline 48) 4-Chlorophenyl-phenylether & 0.4 & 47.0 & -46.6 & 0.3 & 41.8 & -41.5 \\
\hline 49) Fluorens & 89.9 & 72.9 & 17.0 & 58.9 & 62.2 & -3.3 \\
\hline 50) 4-Nitroanlline & 37.9 & 26.1 & 11.8 & 5.4 & 9.9 & -4.5 \\
\hline 51) *Phenanthren $\theta-d 10$ & 100.0 & 400.0 & 0.0 & 100.0 & 100.0 & 0.0 \\
\hline 52) 4,6-Dinitro-2-methylphenol & 88.0 & 85.1 & 2.9 & 70.1 & 97.3 & -27.1 \\
\hline 53) $\mathrm{N}$-Nitrosodiphenylamine & 79.7 & 53.5 & 26.2 & 109.8 & 30.2 & 79.6 \\
\hline 54) \#2,4,6-Tribromophenol & 89.9 & 85.0 & 5.0 & $83 . \overline{3}$ & 92.1 & -8.8 \\
\hline 55) 4-Bromophenyl-phenylether & 89.3 & 71.6 & 17.7 & 63.0 & 67.5 & -4.5 \\
\hline 56) Hexachlorobenzene & 90.3 & 39.9 & 50.4 & 7.5 & 8.9 & -1.4 \\
\hline 57) Pentachloropheriol & 96.4 & 95.2 & 1.2 & 73.9 & 88.5 & -14.7 \\
\hline 58) Phenanthrene & 90.9 & 75.3 & 15.6 & 66.7 & 70.9 & -4.2 \\
\hline 59) Anthracene & 87.9 & 70.9 & 17.0 & 63.5 & 64.5 & -1.1 \\
\hline 60) Di-n-butylphthalate & 5.9 & 3.5 & 2.3 & 8.1 & 8.8 & -0.7 \\
\hline 61) Fluoranthene & 88.0 & 73.7 & 14.3 & 66.9 & 72.7 & -5.8 \\
\hline 62) *Chrysene-d12 & 100.0 & 100.0 & 0.0 & 100.0 & 100.0 & 0.0 \\
\hline 63) Pyrene & 87.1 & 80.0 & 7.1 & 65.0 & 70.2 & -5.2 \\
\hline 64) \#Terphenyl-d14 & 83.7 & 77.1 & 6.6 & 60.0 & 68.8 & -8.8 \\
\hline 65) Butylbenzylphthalate & 5.8 & 3.3 & 2.5 & 6.9 & 8.9 & -2.0 \\
\hline 66) $3,3^{\prime}$-Dichlorobenzidine & 2.7 & 0.0 & 2.7 & 5.1 & 9.8 & -4.7 \\
\hline 67) Benzo(a)anthracene & 89.6 & 81.3 & 8.2 & 65.9 & 70.9 & -5.0 \\
\hline 68) Chrysene & 94.4 & 86.9 & 7.5 & 69.5 & 76.1 & -6.5 \\
\hline 69) Bis (2-Ethylhexyl)phthalate & 37.1 & 66.2 & -29.1 & 7.1 & 10.2 & -3.1 \\
\hline 70) *Perylent -d12 & 100.0 & 100.0 & 0.0 & 100.0 & 100.0 & 0.0 \\
\hline 71) Di-n-octylphthalate & 19.2 & 30.1 & -10.9 & 6.6 & 9.5 & -2.8 \\
\hline 72) Benzo(b)fluoranthene & 87.7 & 76.2 & 11.6 & 61.3 & 66.3 & -5.0 \\
\hline 73) Benzo (k)fluoranthene & 92.1 & 81.2 & 10.9 & 63.0 & 66.7 & -3.7 \\
\hline 74) Benzo(a)pyrene & 86.9 & 76.1 & 10.8 & 60.5 & 64.0 & -3.5 \\
\hline 75) Indeno $(1,2,3-c d)$ pyrene & 90.4 & 76.9 & 13.4 & 61.2 & 69.5 & -8.3 \\
\hline 76) Dibenz $(a, h)$ anthracene & 80.4 & 71.6 & 8.9 & 54.6 & 65.7 & -11.0 \\
\hline 77) Benzo(g,h,l)perylene & 87.3 & 75.2 & 12.2 & 56.5 & 58.9 & -2.5 \\
\hline NPH (Paraffin Hydrocarbon, C12- & 57.4 & 0.1 & 27.3 & 5.7 & & -2.5 \\
\hline
\end{tabular}

* Compound is an Internal Standara

\# Compound is a Surrogate Splke 


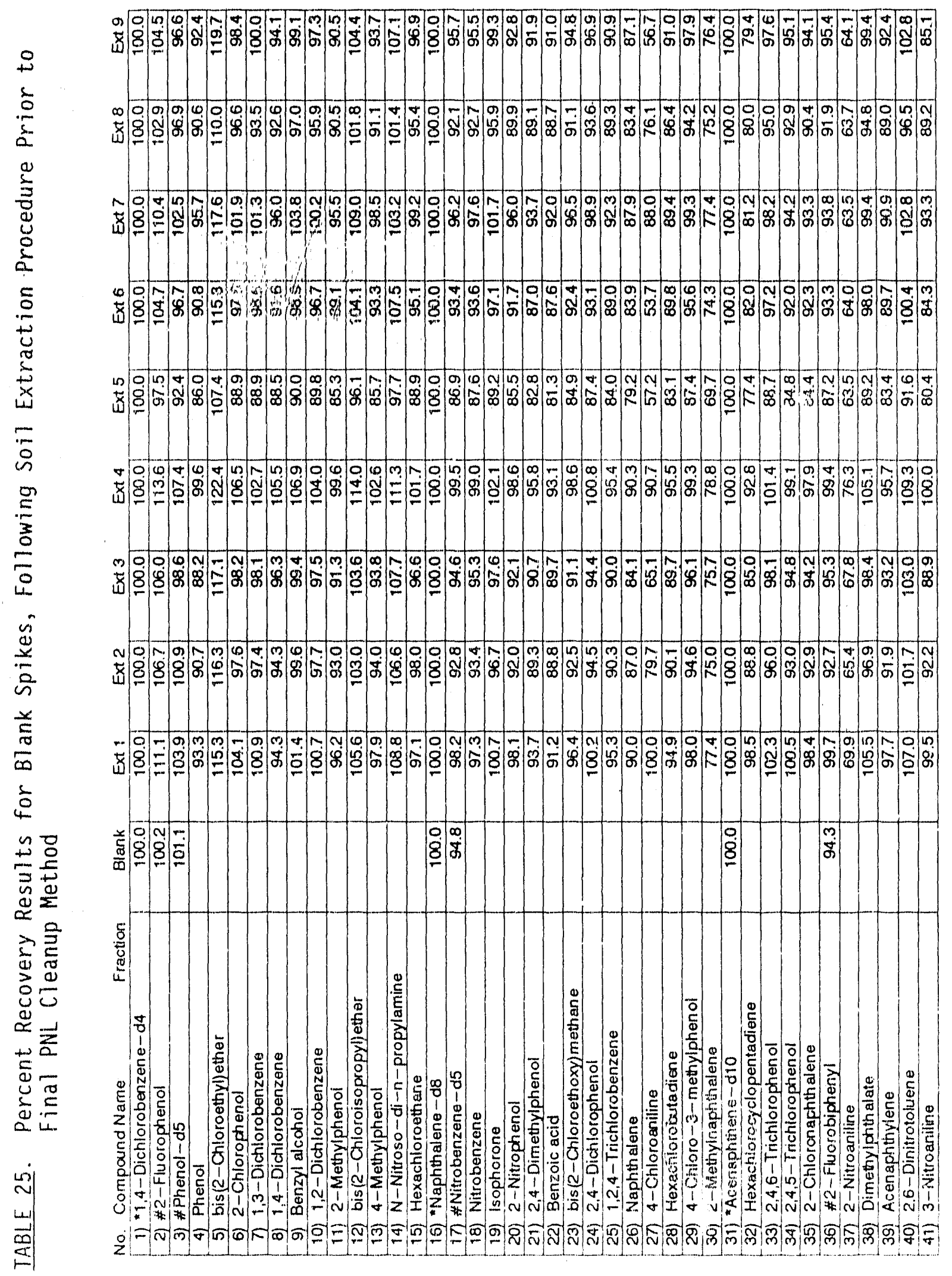




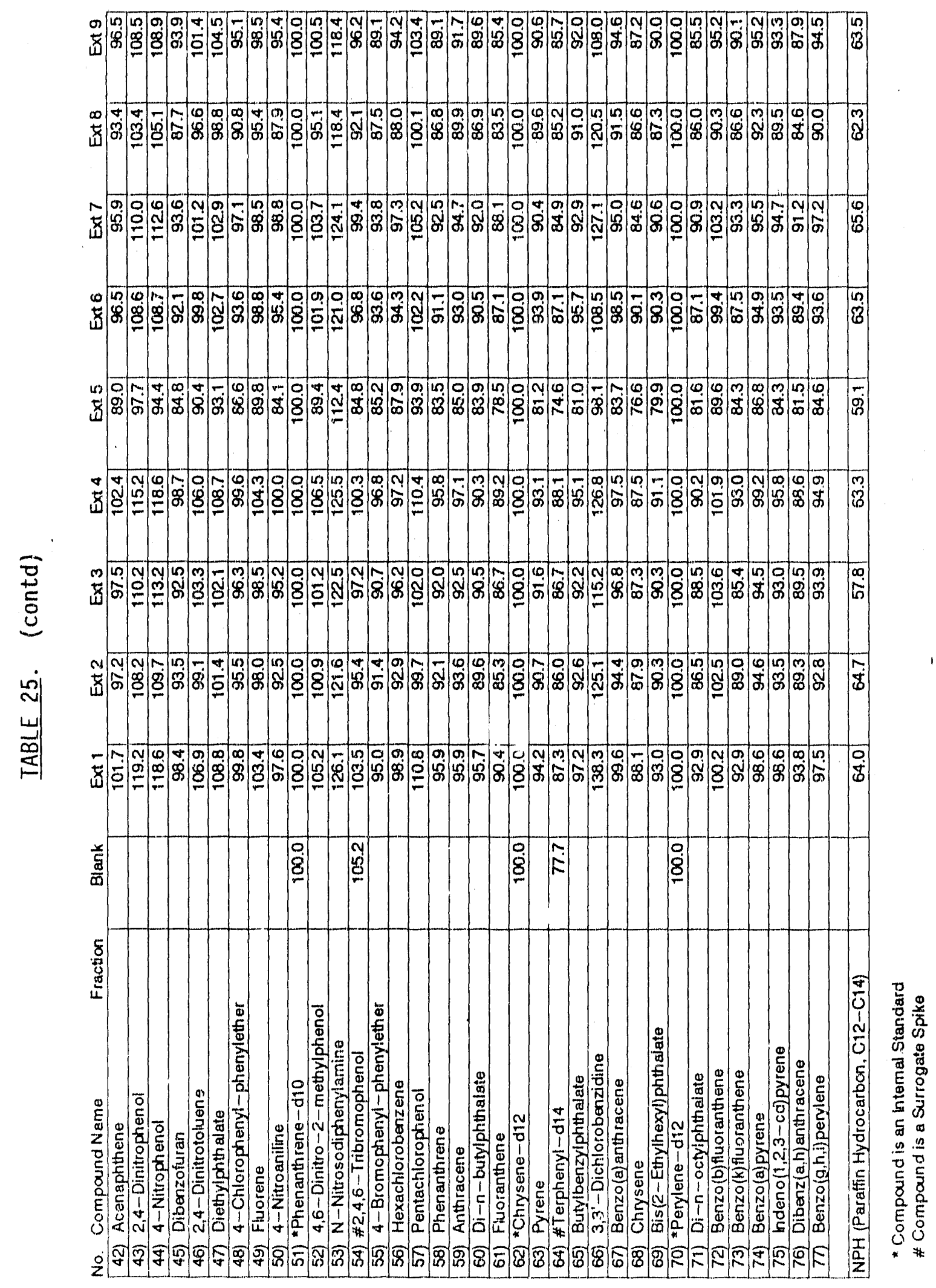


TABLE 26. Percent, Accuracy, and Control Limit Data from Blank Spikes Following Sol1 Extraction Procedure and Prior to Final PNL Cleanup Method

\begin{tabular}{|c|c|c|c|c|}
\hline No. Compound Name & $\begin{array}{r}\text { Mean } \\
\text { Recovery }\end{array}$ & $\mid-1)$ & $-3 \times S T D$ & $+3 \times S T D$ \\
\hline 1) ${ }^{* 1,4-D \mid c h l o r o b e n z e n e-d 4}$ & 100.0 & 0.0 & 100.0 & 100.0 \\
\hline 2) \#2-Fluorophenol & 105.8 & 5.0 & 90.8 & 120.7 \\
\hline 3) \#Phenol-d5 & 99.7 & 4.3 & 86.7 & 112.7 \\
\hline 4) Phenol & 91.9 & 4.0 & 79.9 & 103.9 \\
\hline 5) bis $(2-$ Chloroethyl)ether & 115.7 & 4.6 & & \\
\hline 6) 2-Chlorophenol & 98.9 & 5.0 & 83.8 & 114.0 \\
\hline 7) 1,3-Dichilorobenzene & 97.9 & 4.3 & & \\
\hline 8) 1,4-Dichlorobenzene & 94.8 & 4.7 & 80.8 & 108.8 \\
\hline 9) Benzyl alcohol & 99.6 & 4.7 & & \\
\hline 10) 1,2-Dichlorobenzene & 97.7 & 3.9 & & \\
\hline 11) 2-Methylphenol & 92.3 & 4.3 & & \\
\hline 12) bis(2-Chloroisopropyl)ether & 104.6 & 4.9 & & \\
\hline 13) 4-Methylphenol & 94.5 & 4.8 & & \\
\hline 14) $N$-Nitroso-di-n-propylamine & 105.7 & 4.2 & 93.1 & 118.3 \\
\hline 15) Hexachloroethane & 96.5 & 3.5 & & \\
\hline 16) *Naphthalene - d8 & 100.0 & 0.0 & 100.0 & 100.0 \\
\hline 17) \#Nitrobenzene-d5 & 94.4 & 3.5 & 83.9 & 104.9 \\
\hline 18) Nitrobenzene & 94.7 & 3.4 & & \\
\hline 19) Isophorone & 97.8 & 3.9 & & \\
\hline 20) 2-Nitrophenol & 93.0 & 4.1 & & \\
\hline 21) 2,4-Dimethylphenol & 90.4 & 3.9 & & \\
\hline 22) Benzolic acid & 89.3 & 3.5 & & \\
\hline 23) bis(2-Chloroethoxy)methane & 93.1 & 4.1 & & \\
\hline 24) 2,4-Dichlorophenol & 95.5 & 4.2 & & \\
\hline 25) 1,2,4-Trichlorobenzene & 90.7 & 3.5 & 80.4 & 101.1 \\
\hline 26) Naphthalene & 85.9 & 3.6 & & \\
\hline 27) 4-Chloroaniline & 74.1 & 16.8 & & \\
\hline 28) Hexachlorobutadiene & 90.0 & 3.8 & & \\
\hline 29) 4-Chloro-3-methylphenol & 95.8 & 3.7 & 84.8 & 106.9 \\
\hline 30) 2-Methylnaphthalene & 75.5 & 2.6 & & \\
\hline 31) *Acenaphthene-d10 & 100.0 & 0.0 & 100.0 & 100.0 \\
\hline 32) Hexachlorocyclopentadiene & 85.0 & 7.0 & & \\
\hline 33) 2,4,6-Trichlorophenol & 97.2 & $3 . \overline{9}$ & & \\
\hline 34) 2,4,5-Trichlorophenol & 94.0 & 4.5 & & \\
\hline 35) 2-Chioronaphthalene & 93.1 & 4.1 & & \\
\hline 36) \#2-Fluorobipheny! & 94.3 & 3.6 & 83.4 & 105.2 \\
\hline 37) 2-Nitroaniline & 66.5 & 4.3 & & \\
\hline 38) Dimethylphthalate & 98.5 & 5.0 & & \\
\hline 39) Acenaphthylene & 91.5 & 4.1 & & \\
\hline 40) 2,6-Dinitrotoluene & 101.7 & 5.2 & & \\
\hline 41) 3-Nitroaniline & 90.3 & 6.7 & & \\
\hline
\end{tabular}


TABLE 26. (contd)

\begin{tabular}{|c|c|c|c|c|}
\hline No. Compound Name & $\begin{array}{l}\text { Mean } \\
\text { Recovery }\end{array}$ & $v-1)$ & $-3 \times S T D$ & $+3 \times S T D$ \\
\hline 42) Acenaphthene & 96.7 & 4.0 & 84.6 & 108.7 \\
\hline 43) 2,4-DInitrophenol & 109.0 & 6.2 & & \\
\hline 44) 4-Nitrophenol & 110.0 & 7.4 & 87.9 & 132.0 \\
\hline 45) Dibenzofuran & 92.8 & 4.5 & & \\
\hline 46) $2,4 \ldots$ Dinitrotoluene & 100.5 & 5.0 & 85.5 & 115.5 \\
\hline 47) Diethylphthalate & 102.5 & 4.8 & & \\
\hline 48) 4-Chlorophenyl-phenylether & 94.9 & 4.2 & & \\
\hline 49) Fluorene & 98.3 & 4.2 & & \\
\hline 50) 4-Nitroaniline & 94.1 & 5.2 & & \\
\hline 51) *Phenanthrene-d10 & 100.0 & 0.0 & 100.0 & 100.0 \\
\hline 52) 4,6 -Dlinitro-2-methylphenol & 100.5 & 5.3 & & \\
\hline 53) $\mathrm{N}$-Nitrosodiphenylamine & 121.1 & 4.3 & & \\
\hline 54) \#2,4,6-Tribromophenol & 97.1 & 5.8 & 79.7 & 114.5 \\
\hline 55) 4-Bromophenyl-phenylether & 91.4 & 3.7 & & \\
\hline 56) Hexachlorobenzene & 94.1 & 3.9 & & \\
\hline 57) Pentachlorophenol & 103.1 & 5.3 & 87.2 & 119.0 \\
\hline 58) Phenanthrene & 91.0 & 4.0 & & \\
\hline 59) Anthracene & 92.6 & 3.6 & & \\
\hline 60) Di-n-butylphthalate & 89.9 & 3.2 & & \\
\hline 61) Fluoranthene & 86.0 & 3.5 & & \\
\hline 62) *Chrysene-d12 & 100.0 & 0.0 & 100.0 & 100.0 \\
\hline 63) Pyrene & 90.6 & 3.9 & 78.9 & 102.2 \\
\hline 64) \#Terphenyl-d14 & 84.3 & 4.5 & 70.9 & 97.7 \\
\hline 65) Butylbenzylphthalate & 92.2 & 4.6 & & \\
\hline 66) $3,3^{\prime}$-Dichlorobenzidine & 118.6 & 12.4 & & \\
\hline 67) Benzo(a)anthracene & 94.6 & 4.8 & & \\
\hline 68) Chrysene & 86.2 & 3.9 & & . \\
\hline 69) Bis(2-Ethylhexyl)phthalate & 89.2 & 3.8 & & \\
\hline 70) *Perylene-d12 & 100.0 & 0.0 & 100.0 & 100.0 \\
\hline 71) Di-n-octylphthalate & 87.7 & 3.4 & & \\
\hline 72) Benzo(b) fluoranthene & 98.4 & 5.4 & & \\
\hline 73) Benzo(k)fluoranthene & 89.1 & 3.4 & & \\
\hline 74) Benzo(a)pyrene & 94.6 & 3.6 & & \\
\hline 75$)$ Indeno $(1,2,3$-cd)pyrene & 92.9 & 4.0 & & \\
\hline 76) Dibenz $(a, h)$ anthracene & 88.4 & 3.6 & & \\
\hline 77) Benzo $(g, h, i)$ perylene & 93.2 & 4.0 & & \\
\hline NPH (Paraffin Hydrocarbon, $\mathrm{C} 12-\mathrm{C} 14)$ & 62.6 & 2.6 & & \\
\hline
\end{tabular}

* Compound is an Internal Standard

\# Compound is a Surrogate Spike 


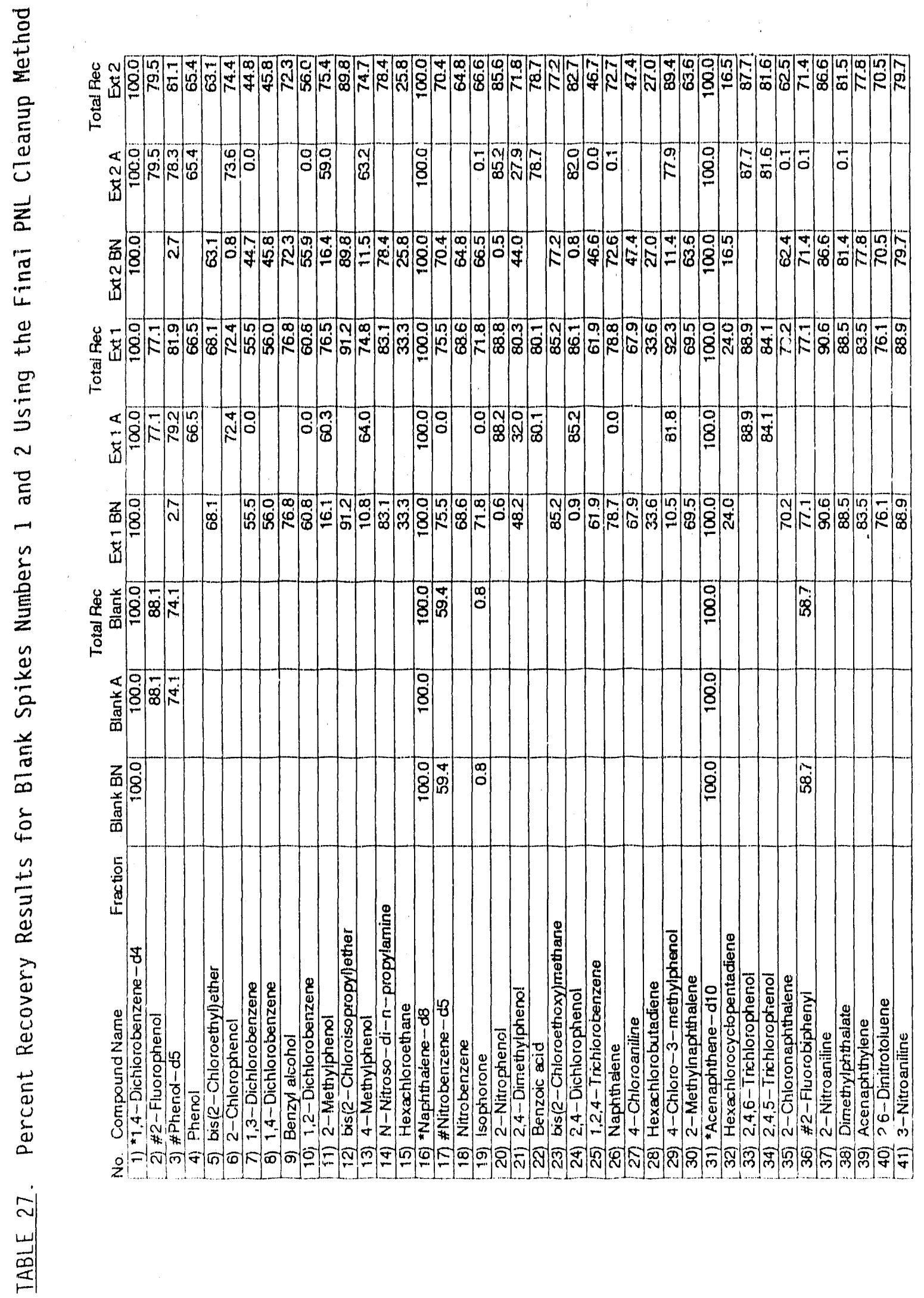




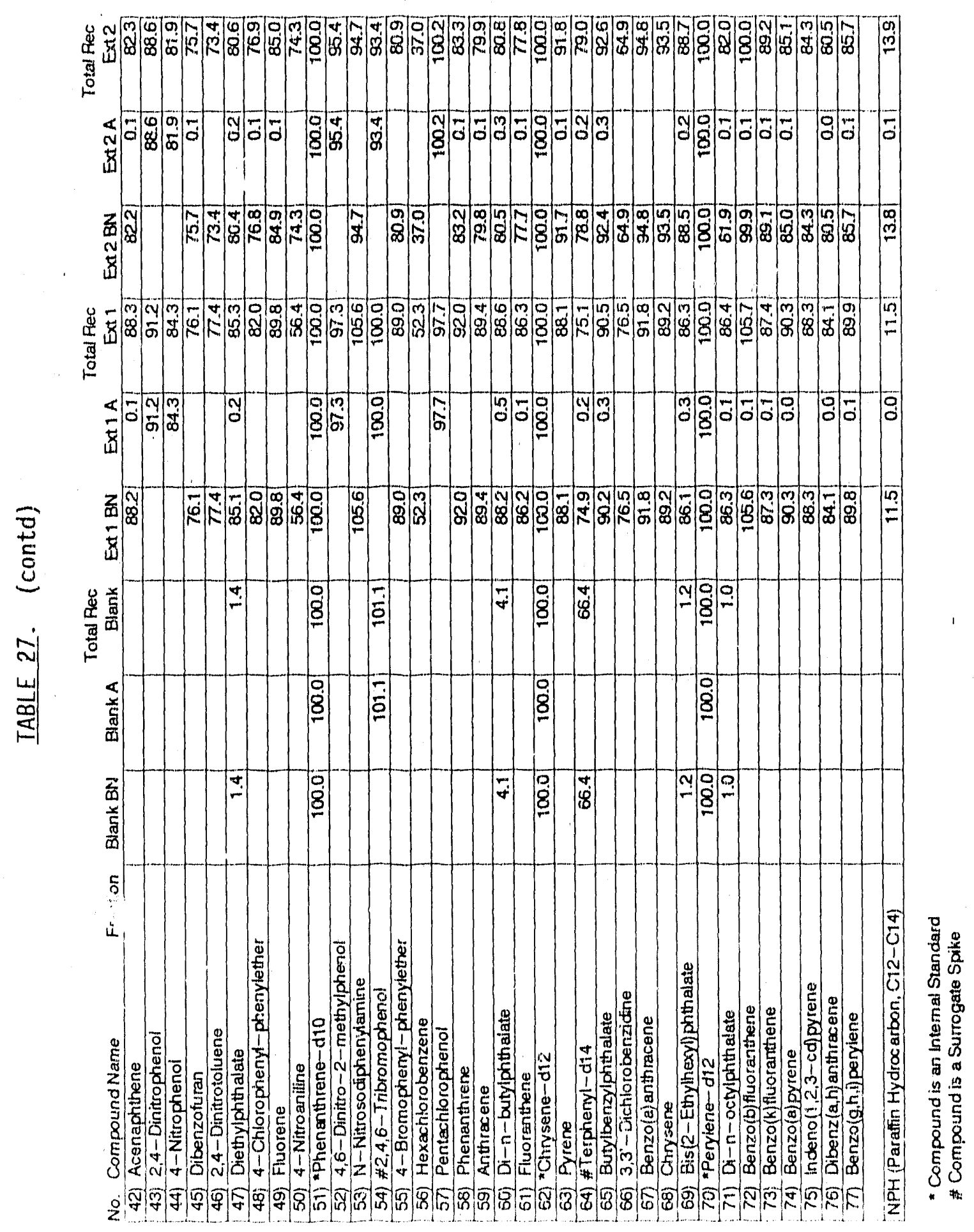




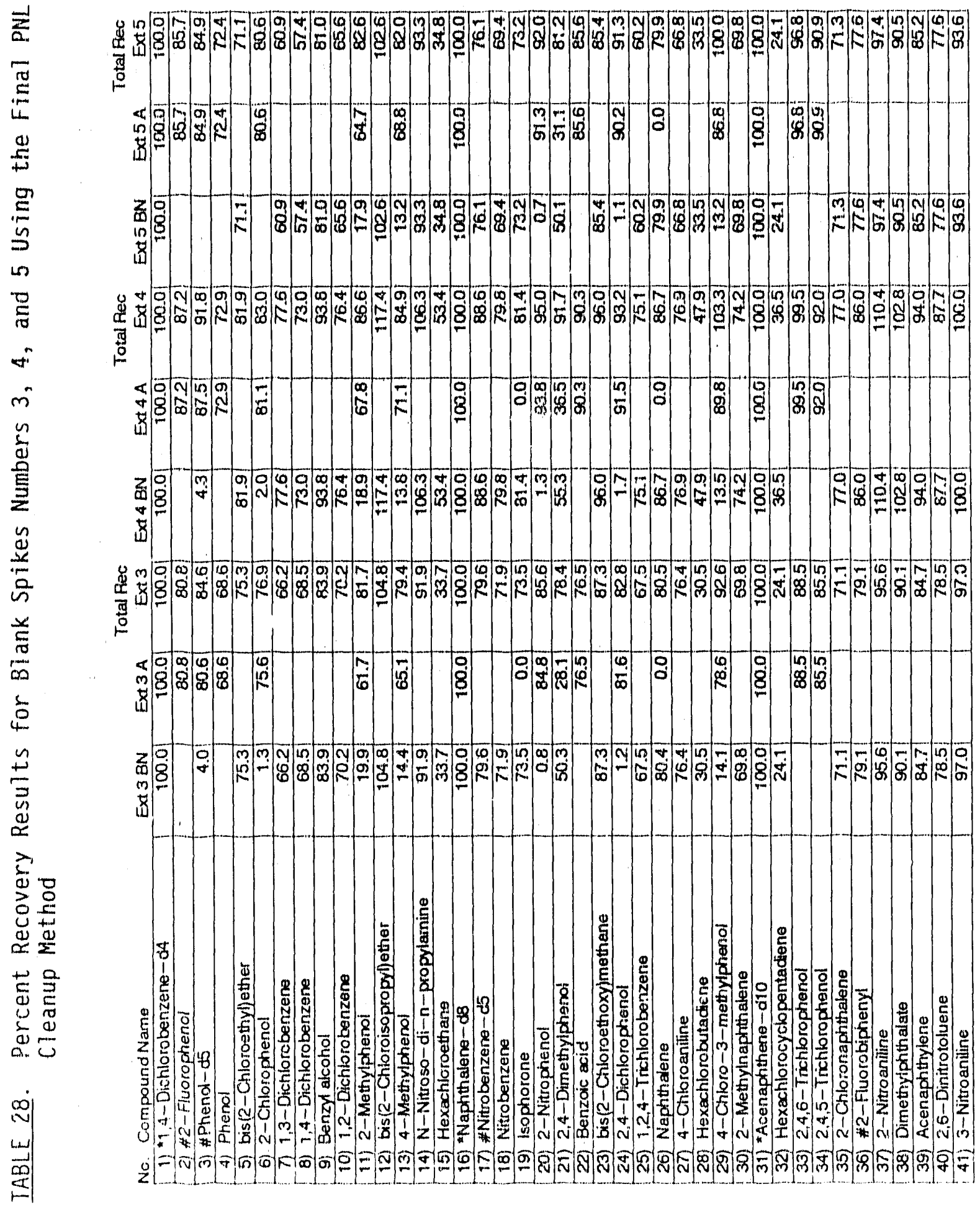



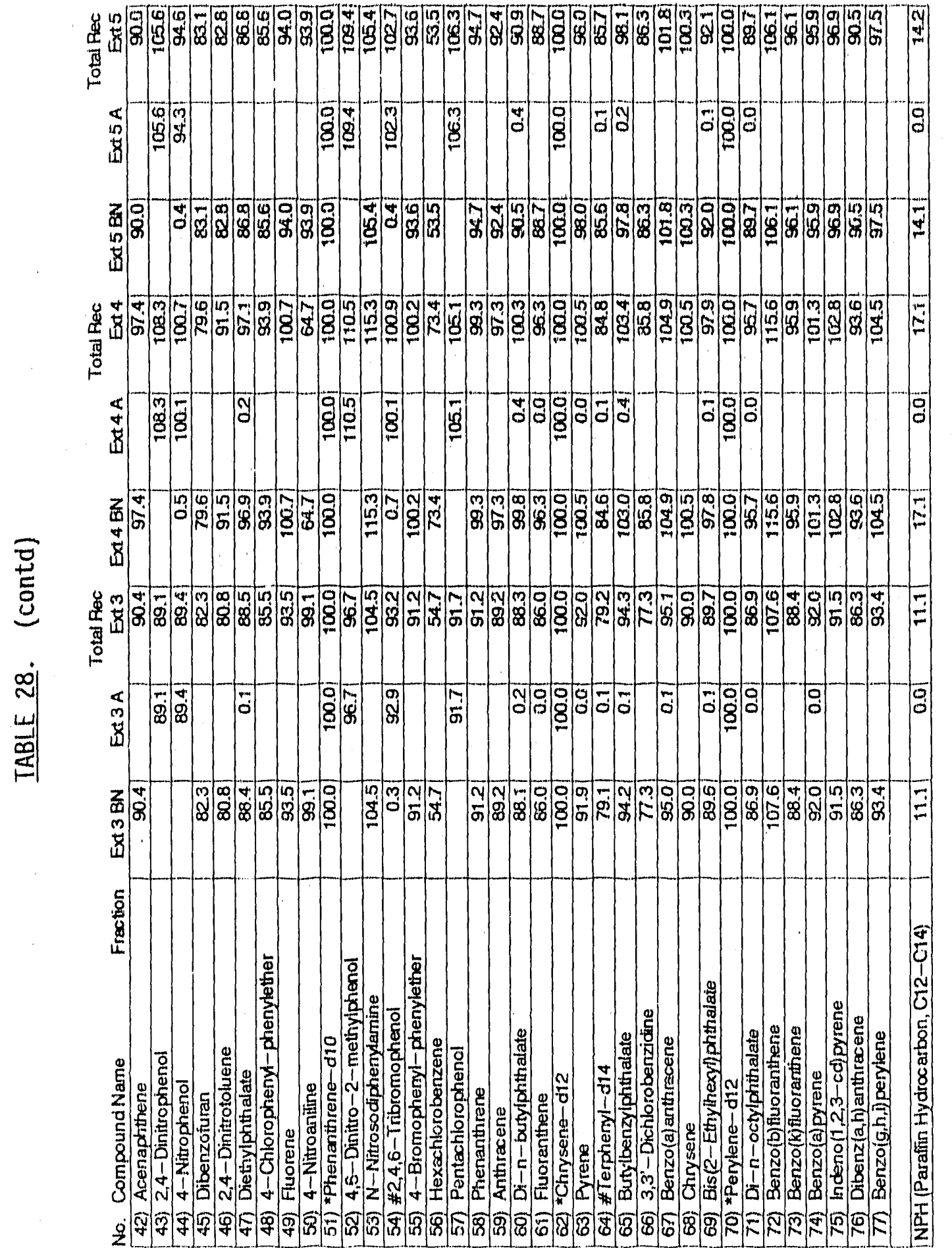
焉

量

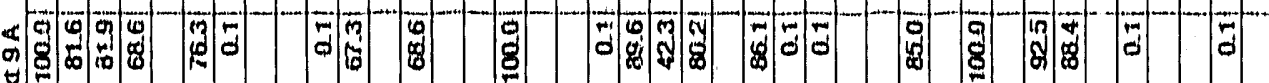
㟔

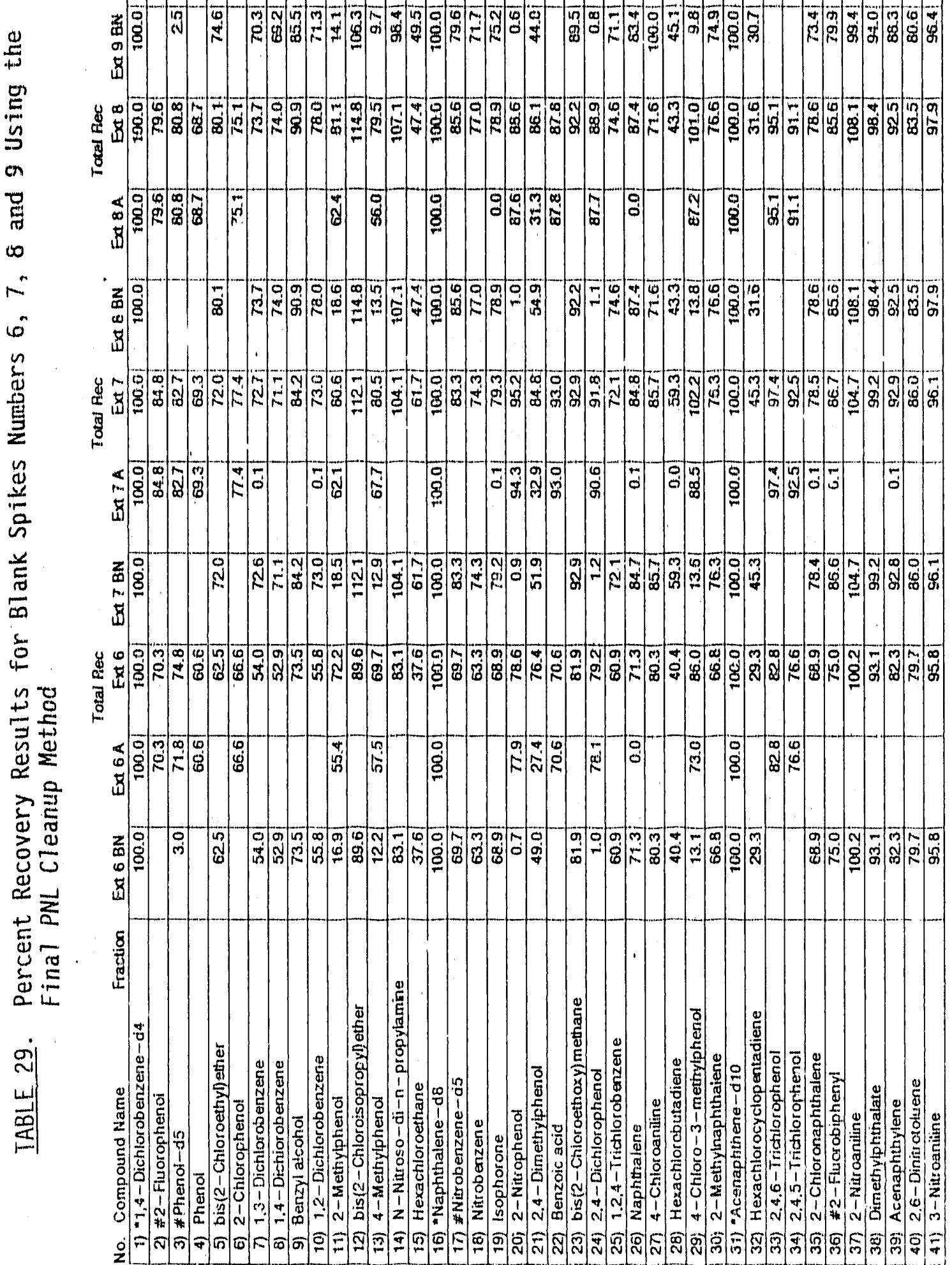


造

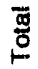

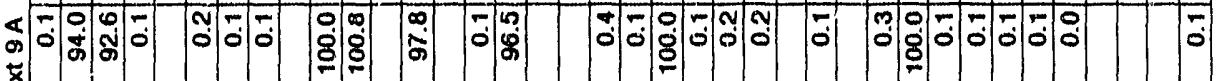

岗.

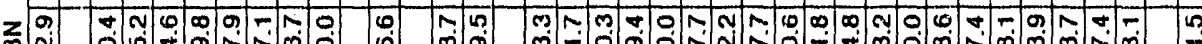

竞

9 0 - 0 m

$\underset{0}{0}$

这

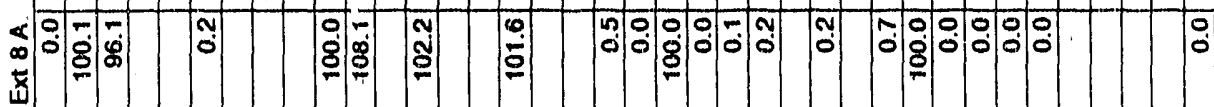

z nீ m

茨.

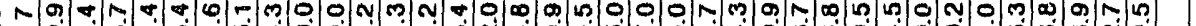

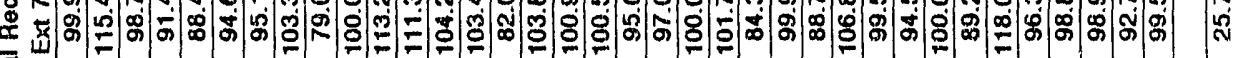

可

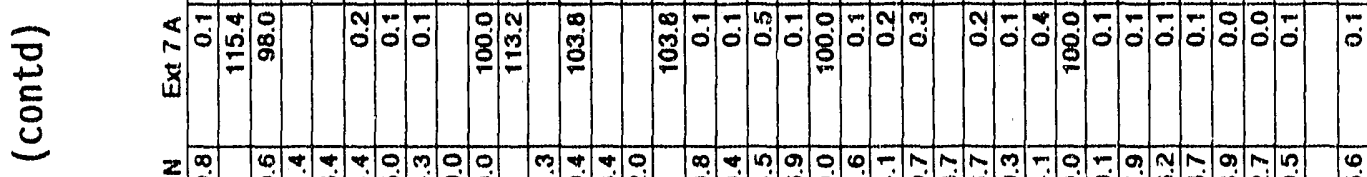

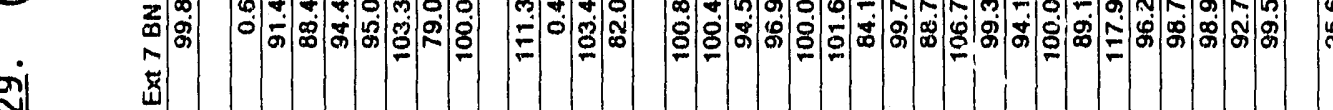

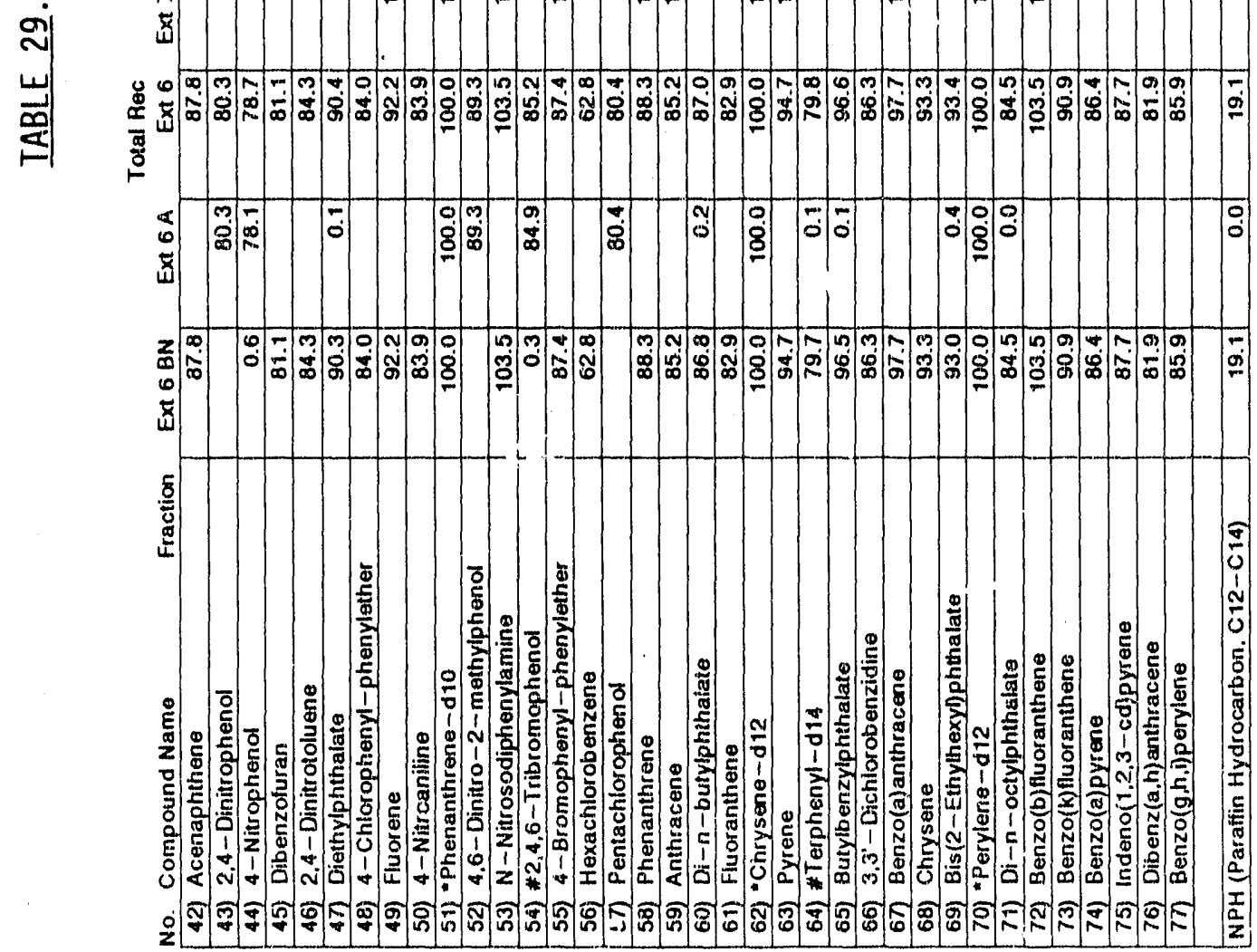

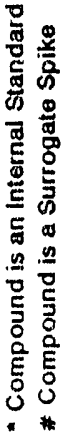


TABLE 30. Precision Accuracy, and Control Limit Data from Blank Spikes Using the Final PNL Cleanup Method

\begin{tabular}{|c|c|c|c|c|}
\hline No. Compound Name & $\begin{array}{r}\text { Mean } \\
\text { Recovery }\end{array}$ & STD $(N-1)$ & $-3 \times S T D$ & $+3 \times S T D$ \\
\hline 1) *1,4-Dichlorobenzene-d4 & 100.0 & 0.0 & 100.0 & 100.0 \\
\hline 2) \#2-Fluorophenol & 81.5 & 5.3 & 65.4 & 97.5 \\
\hline 3) \#Phenol-d5 & 82.1 & 5.1 & 66.8 & 97.4 \\
\hline 4) Phenol & 68.1 & 3.7 & 56.9 & 79.3 \\
\hline 5) bis $(2-$ Chloroethylether & 72.1 & 6.8 & & \\
\hline 6) 2--Chlorophenol & 75.9 & 4.7 & 61.7 & 90.0 \\
\hline 7) 1,3-Dichlorobenzene & 64.0 & 10.9 & & \\
\hline 8) 1,4-Dichlorobenzene & 63.1 & 10.2 & 32.5 & 93.7 \\
\hline 9) Benzyl alcohol & 82.4 & 7.3 & & \\
\hline 10) 1,2 -Dichlorobenzene & 67.5 & 8.4 & & \\
\hline 11) 2-Methylphenol & 79.8 & 4.3 & & \\
\hline 12) bis(2-Chloroisopropyl)ether & 103.2 & 10.8 & & \\
\hline 13) 4-Methylphenol & 78.2 & 4.5 & & \\
\hline 14) N-Nitroso-di-n-propylamine & 94.0 & 10.8 & 61.7 & 126.3 \\
\hline 15) Hexachloroethane & 41.9 & 11.6 & & \\
\hline 16) *Naphthalene - d8 & 100.0 & 0.0 & 100.0 & 100.0 \\
\hline 17) \#Nitrobenzene-d5 & 76.8 & 8.6 & 50.9 & 102.7 \\
\hline 18) Nitrobenzene & 71.2 & 5.4 & & \\
\hline 19) Isophorone & 74.3 & 4.9 & & \\
\hline 20) 2-Nitrophenol & 88.8 & 5.2 & & \\
\hline 21) 2,4-Dimethylphenol & 81.9 & 6.0 & & \\
\hline 22) Benzoic acid & 82.5 & 7.2 & & \\
\hline 23) bis(2-Chloroethoxy)methane & 87.5 & 5.9 & & \\
\hline 24) 2,4-Dichlorophenol & 87.0 & 4.8 & & \\
\hline 25) 1,2,4-Trichlorobenzene & 65.6 & 9.2 & 38.1 & 93.1 \\
\hline 26) Naphthalene & 80.6 & 5.7 & & \\
\hline 27) 4-Chloroaniline & 74.8 & 14.4 & & \\
\hline 28) Hexachlorobutadiene & 40.1 & 10.1 & & \\
\hline 29) 4-Chloro-3-methylphenol & $\overline{95.7}$ & 6.1 & 77.3 & 114.1 \\
\hline 30) 2-Methylnaphthalene & 71.3 & 4.5 & & \\
\hline 31) *Acenaphthene - -d10 & 100.0 & 0.0 & 100.0 & 100.0 \\
\hline 32) Hexachlorocyclopentadiene & 29.1 & 8.4 & & \\
\hline 33) 2,4,6-Trichlorophenol & 92.1 & 5.5 & & \\
\hline 34) 2,4,5-Trichlorophenol & 87.0 & 5.4 & & \\
\hline 35) 2-Chloronaphthalene & 72.4 & 5.2 & & \\
\hline 36) \#2-Fluorobiphenyl & 77.7 & 8.3 & 52.7 & 102.7 \\
\hline 37) 2-Nitroaniline & 99.2 & 7.8 & & \\
\hline 38) Dimethylphthalate & 93.1 & 6.4 & & \\
\hline 39) Acenaphthylene & 86.8 & 5.5 & & \\
\hline 40) 2,6-Dinitrotoluene & 80.0 & 5.3 & & \\
\hline 41) 3-Nitroaniline & 93.9 & 6.2. & & \\
\hline
\end{tabular}


TABLE 30. (contd)

No. Compound Name

Recovery STD $(\mathrm{N}-1)$-3XSTD +3XSTD

42) Acenaphthene

43) 2,4-Dinitrophenol

44) 4-Nitrophenol

45) Dibenzofuran

4E) 2,4-Dinitrotoluene

47) Diethylphthalate

48) 4-Chlorophenyi-phenylether

49) Fluorene

50) 4-Nitroaniline

51) *Phenanthrene-d10

52) 4,6-Dinitro-2-methylphenol

53) $\mathrm{N}$-Nitrosodiphenylamine

54) \#2,4,6-Tribromophenol

55) 4-Bromophenyl-phenylether

56) Hexachlorobenzene

57) Pentachlorophenol

58) Phenanthrene

59) Anthracene

60) Di-n-butylphthalate

61) Fivoranthene

62) *Chrysene-d12

63) Pyrene

64) \#Terphenyl-d14

65) Butylbenzylphthalate

66) 3,3'-Dichlorobenzidine

67) Benzo(a)anthracene

68) Chrysene

69) Bis(2-Ethylhexyl)phthalate

70) *Perylene-d12

71) Di-n-octylphthalate

72. Benzo(b)flucranthene

73) Benzo(k)fluoranthene

74) Benzo(a)pyrene

75) Indeno $(1,2,3$-cd)pyrene

76) Dibenz $(a, h)$ anthracene

77) Benzo $(g, h, i)$ perylene

NPH (Paraffin Hydrocarbon, C12-C14)

91.9

97.0

90.9

81.7

83.5

89.6

87.1

95.4

80.6

100.0

102.3

106.4

98.1

93.1

61.2

98.1

93.5

91.3

90.7

88.7

100.0

97.0

81.0

98.0

82.8

101.0

96.8

93.0

100.0

88.3

108.7

91.5

93.7

93.8

87.8

94.4

16.8

\begin{tabular}{|r|r|r|}
\hline 5.7 & 74.9 & 108.9 \\
\hline 11.2 & & \\
\hline 7.8 & 67.6 & 114.2 \\
\hline 6.0 & & \\
\hline 5.7 & 66.4 & 100.6 \\
\hline
\end{tabular}

5.1

6.1

6.2

15.2

\begin{tabular}{rr|r}
\hline 0.0 & 100.0 & 100.0
\end{tabular}

8.3

5.8

\begin{tabular}{|l|l|l|}
\hline 5.9 & 80.4 & 115.8 \\
\hline
\end{tabular}

6.9

13.4

8.1

5.6

6.4

5.6

6.3

\begin{tabular}{|r|r|r|}
\hline 0.0 & 100.0 & 100.0 \\
\hline 6.2 & 78.5 & 115.5 \\
\hline 7.1 & 59.6 & 102.
\end{tabular}

\begin{tabular}{|l|l|l|}
\hline 7.1 & 59.6 & 102.4 \\
\hline
\end{tabular}

5.5

8.3

6.9

6.4

4.5

$\begin{array}{lll}0.0 & 100.0 & 100.0\end{array}$

4.1

6.0
3.7

3.7

$\frac{5.7}{6.4}$

5.0

4.8

* Compound is an Internal Standard

\# Compound is a Surrogate Spike 
TABLE 31. Recovery Loss from the Use of Final PNL Cleanup Procedure

\begin{tabular}{|c|c|c|c|c|}
\hline No. Compound Name & $\begin{array}{r}\text { Mean Before } \\
\text { Cleanup }\end{array}$ & $\begin{array}{r}\text { Mean After } \\
\text { Cleanup }\end{array}$ & Difference & $\begin{array}{l}\text { Percent } \\
\text { Change }\end{array}$ \\
\hline 1) *1,4-Dichloroberizene-d4 & 100.0 & 100.0 & 0.0 & 0.0 \\
\hline 2) \#2-Fluorophenol & 105.8 & 81.5 & -24.3 & -23.0 \\
\hline 3) APhenol-d5 & 99.7 & 82.1 & -17.6 & -17.7 \\
\hline 4) Phenol & 91.9 & 68.1 & -23.8 & -25.9 \\
\hline 5) bis(2-Chloroethyl)ether & 115.7 & 72.1 & -43.6 & -37.7 \\
\hline 6) 2-Chlorophenol & 98.9 & 75.9 & -23.0 & -23.3 \\
\hline 7) 1,3-Dichlorobenzene & 97.9 & 64.0 & -34.0 & -34.7 \\
\hline 8) 1,4-Dichlorobenzene & 94.8 & 63.1 & -31.7 & -33.4 \\
\hline 9) Benzyl alcohol. & 99.6 & 82.4 & -17.1 & -17.2 \\
\hline 10) 1,2-Dichlorobenzene & 97.7 & 67.5 & -30.3 & -31.0 \\
\hline 11) 2-Methylphenol & 92.3 & 79.8 & -12.5 & -13.6 \\
\hline 12) bis(2-Chloroisopropyl 3 ther & 104.6 & 103.2 & -1.5 & -1.4 \\
\hline 13) 4-Methylphenol & 94.5 & 78.2 & -16.3 & -17.3 \\
\hline 14) N-Nitroso-di-n-propylamine & 105.7 & 94.0 & -11.7 & $-\$ 1.1$ \\
\hline 15) Hexachloroethane & 96.5 & 41.9 & -54.6 & -56.6 \\
\hline 16) *Naphthalene-d8 & 100.0 & 100.0 & 0.0 & 0.0 \\
\hline 17) \#Nitrobenzene-d5 & 94.4 & 76.8 & -17.6 & -18.7 \\
\hline 18) Nitrobenzene & 94.7 & 71.2 & -23.5 & -24.8 \\
\hline 19) Isophorone & 97.8 & 74.3 & -23.5 & -24.0 \\
\hline 20) 2-Nitrophenol & 93.0 & 88.8 & -4.1 & -4.4 \\
\hline 21) 2,4-Dimethylphenol & 90.4 & 81.9 & -8.5 & -9.4 \\
\hline 22) Benzoic acid & 89.3 & 82.5 & -6.7 & -7.5 \\
\hline 23) bis (2-chloroethoxy)methan $\theta$ & 93.1 & 87.5 & -5.7 & -6.1 \\
\hline 24) 2,4-Dichlorophenol & 95.5 & 87.0 & -8.5 & -8.9 \\
\hline 25) 1,2,4-Trichlorobenzene & 90.7 & 65.6 & -25.1 & -27.7 \\
\hline 26) Naphthalene & 85.9 & 80.6 & -5.2 & -6.1 \\
\hline 27) 4-Chloroaniline & 74.1 & 74.8 & 0.7 & $0 . \dot{9}$ \\
\hline 28) Hexachlorobutadiene & 90.0 & 40.1 & -49.9 & -55.5 \\
\hline 29) 4-Chloro-3-methylphenol & 95.8 & 95.7 & -0.1 & -0.1 \\
\hline 30) 2-Methylnaphthalene & 75.5 & 71.3 & -4.3 & -5.6 \\
\hline 31) *Acenaphthene-d10 & 100.0 & 100.0 & 0.0 & 0.0 \\
\hline 32) Hexachlorocyclopentadiene & 85.0 & 29.1 & -55.9 & -65.8 \\
\hline 33) 2,4,6-Trichlorophenol & 97.2 & 92.1 & -5.0 & -5.2 \\
\hline 34) 2,4,5-Trichlorophenol & 94.0 & 87.0 & -7.1 & -7.5 \\
\hline 35) 2-Chloronaphthalene & 93.1 & 72.4 & -20.7 & -22.2 \\
\hline 36) \#2-Fluorobiphenyl & 94.3 & 77.7 & -16.6 & -17.6 \\
\hline 37) 2-Nitroaniline & 66.5 & 99.2 & 32.8 & 49.3 \\
\hline 38) Dimethylphthalate & 98.5 & 93.1 & -5.4 & -5.5 \\
\hline 39) Acenaphthylene & 91.5 & 86.8 & -4.7 & -5.2 \\
\hline 40) 2,6-Dinitrotoluene & 101.7 & 80.0 & -21.7 & -21.3 \\
\hline 41) 3-Nitroaniline & 90.3 & 93.9 & 3.6 & 4.0 \\
\hline
\end{tabular}


TABLE 31. (contd)

\begin{tabular}{|c|c|c|c|c|}
\hline No. Compound Name & $\begin{array}{r}\text { Mean Before } \\
\text { Cleanupp } \\
\end{array}$ & $\begin{array}{r}\text { Mean After } \\
\text { Cleanup }\end{array}$ & Difference & $\begin{array}{l}\text { Percent } \\
\text { Change }\end{array}$ \\
\hline 42) Acenaphthene & 96.7 & 91.9 & -4.8 & -5.0 \\
\hline 43) 2,4-Dinitrophenol & 109.0 & 97.0 & -12.0 & -11.0 \\
\hline 44) 4-Nitrophenol & 110.0 & 90.9 & -19.1 & -17.4 \\
\hline 45) Dibenzofuran & 92.8 & 81.7 & -11.1 & -12.0 \\
\hline 46) 2,4-Dinitrotoluene & 100.5 & 83.5 & -17.0 & -16.9 \\
\hline 47) Diethylphthalate & 102.5 & 89.6 & -12.9 & -12.6 \\
\hline 48) 4-Chlorophenyl-phenylether & 94.9 & 87.1 & -7.8 & -8.2 \\
\hline 49) Fluorene & 98.3 & 95.4 & -2.9 & -3.0 \\
\hline 50) 4-Nitroaniline & 94.1 & 80.6 & -13.5 & -14.4 \\
\hline 51) *Phenanthrene-d10 & 100.0 & 100.0 & 0.0 & 0.0 \\
\hline 52) 4,6-Dinitro-2-methylphenol & 100.5 & 102.3 & 1.8 & 1.8 \\
\hline 53) $\mathrm{N}$-Nitrosodiphenylamine & 121.1 & 106.4 & -14.7 & -12.1 \\
\hline 54) \#2,4, 6-Tribromophenol & 97.1 & 98.1 & 1.0 & 1.1 \\
\hline 55) 4-Bromophenyl-phenylether & 91.4 & 93.1 & 1.6 & 1.8 \\
\hline 56) Hexachlorobenzene & 94.1 & 61.2 & -32.8 & $-34,9$ \\
\hline 57) Pentacinlorophenol & 103.1 & 98.1 & -4.9 & -4.8 \\
\hline 58) Phenanthrene & 91.0 & 93.5 & 2.5 & 2.7 \\
\hline 59) Anthracene. & 92.6 & 91.3 & -1.2 & -1.3 \\
\hline 60) Di-n-butyiphthalate & 89.9 & 90.7 & 0.8 & 0.9 \\
\hline 61) Fluoranthene & 86.0 & 88.7 & 2.7 & 3.1 \\
\hline 62) *Chrysene-d12 & 100.0 & 100.0 & 0.0 & 0.0 \\
\hline 63) Pyrene & 90.6 & 97.0 & 6.4 & 7.1 \\
\hline 64) \#Terphenyl-d14 & 84.3 & 81.0 & -3.3 & -3.9 \\
\hline 65) Butylbenzylphthalate & 92.2 & 98.0 & 5.8 & 6.3 \\
\hline 66) 3,3'-Dichlorobenzidine & 118.6 & 82.8 & -35.8 & -30.2 \\
\hline 67) Benzo(a)anthracene & 94.6 & 101.0 & 6.3 & 6.7 \\
\hline 68) Chrysene & 86.2 & 96.8 & 10.5 & 12.2 \\
\hline 69) Bis(2-Ethylhexyl)phthalate & 89.2 & 93.0 & 3.8 & 4.3 \\
\hline 70) *Perylene-d12 & 100.0 & 100.0 & 0.0 & 0.0 \\
\hline 71) Di-n-octylphthalate & 87.7 & 88.3 & 0.6 & 0.7 \\
\hline 72) Benzo(b)fluoranthene & 98.4 & 108.7 & 10.3 & 10.4 \\
\hline 73) Benzo(k)fluoranthene & 89.1 & 91.5 & 2.4 & 2.7 \\
\hline 74) Benzo(a)pyrene & 94.6 & 93.7 & -0.9 & -1.0 \\
\hline 75) Indeno $(1,2,3-c d)$ pyrene & 92.9 & 93.8 & 0.9 & 1.0 \\
\hline 76) Dibenz $(a, h)$ anthracene & 88.4 & 87.8 & -0.6 & -0.7 \\
\hline 77 Benzo $(g, h, i)$ perylene & 93.2 & 94.4 & 1.2 & 1.3 \\
\hline NPH (Paraffin Hydrocarbon, $\mathrm{C} 12-\mathrm{C}$ & 62.6 & 16.8 & -45.8 & -73.2 \\
\hline
\end{tabular}

* Compound is an Internal Standard

\# Compound is a Surrogate Spike 

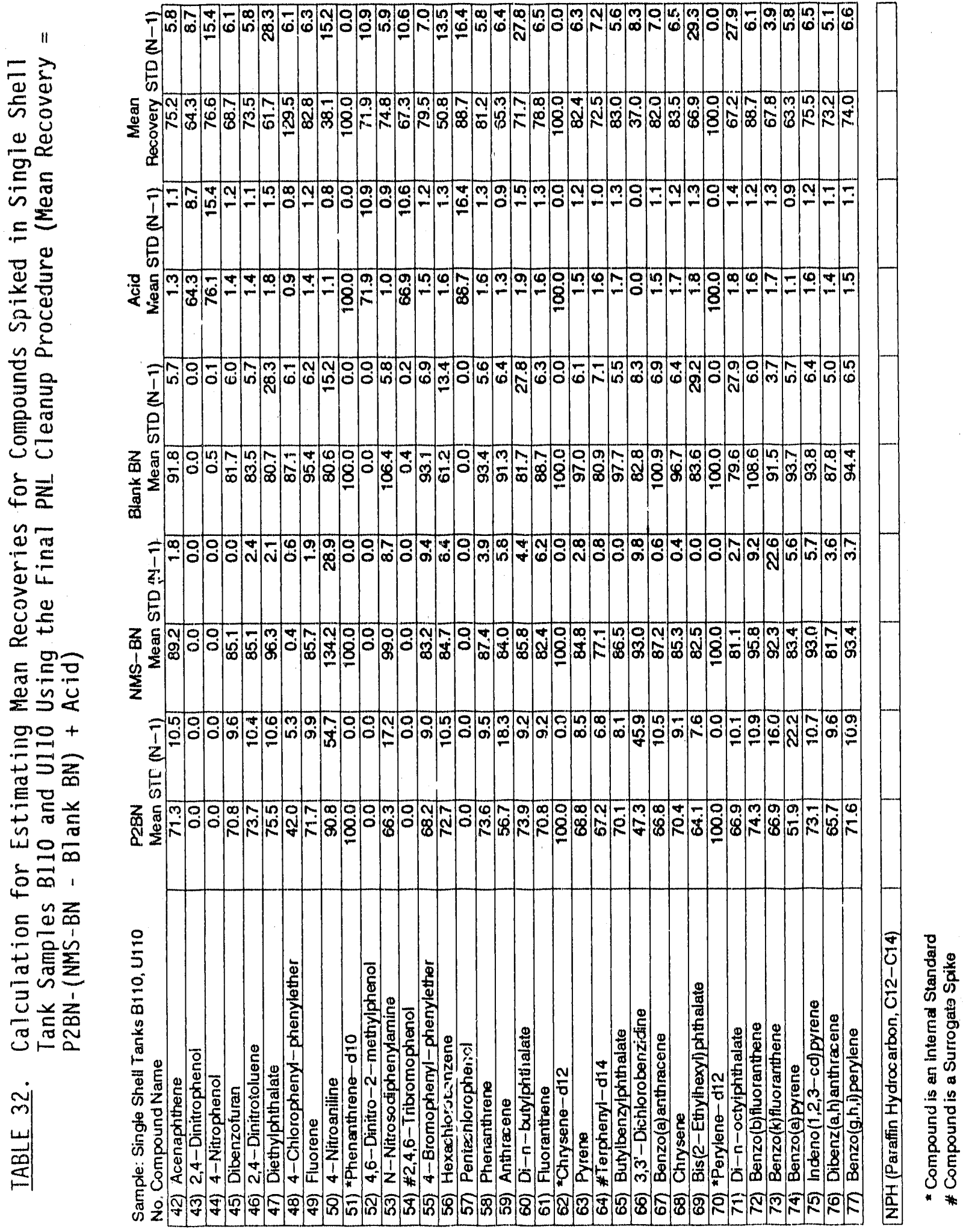


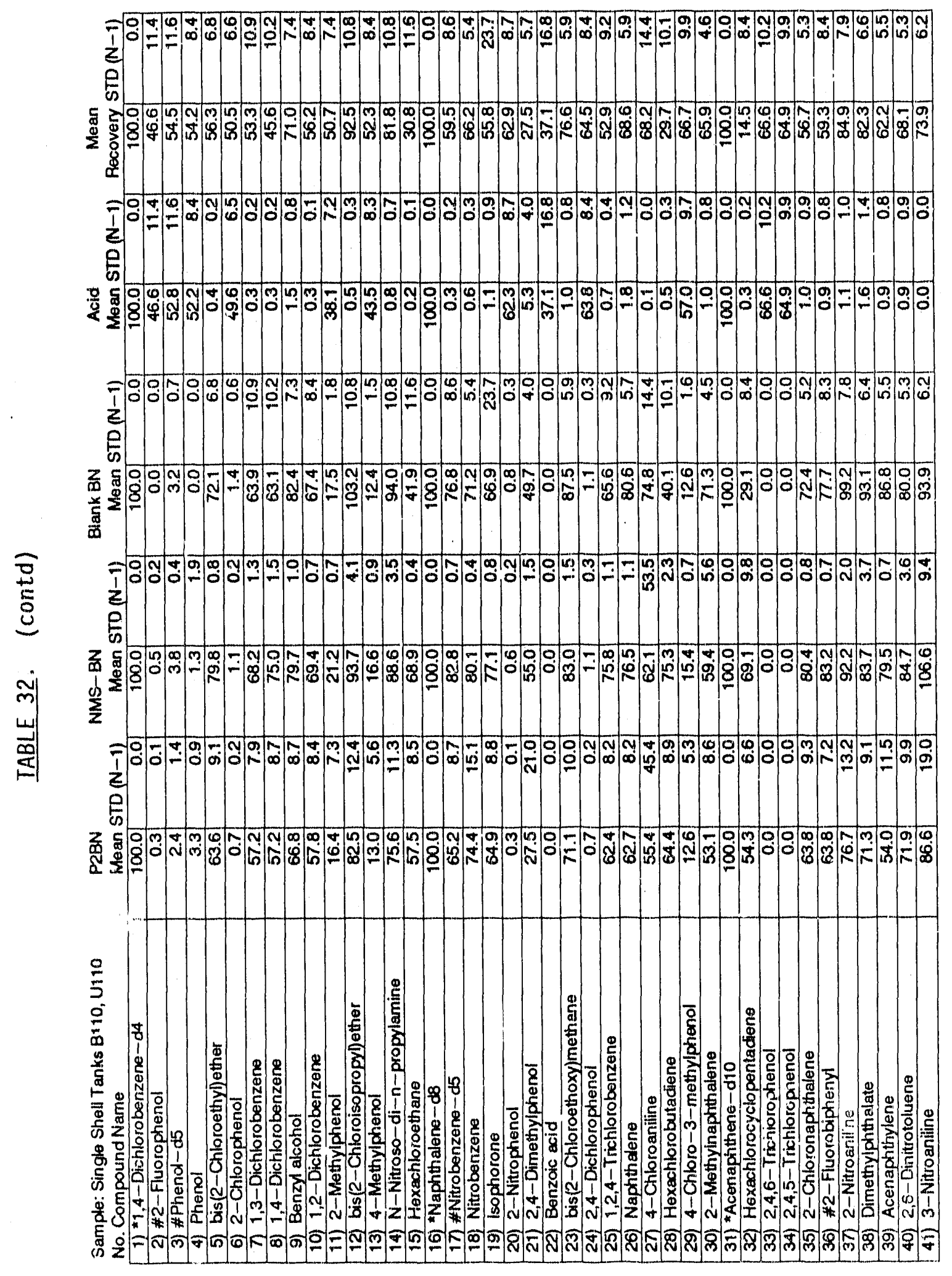


TABLE 33. Estimated Precision, Accuracy and Control Limits for SST Semivolatile Organic Analysis

\begin{tabular}{|c|c|c|c|c|}
\hline $\begin{array}{l}\text { Sample: Single Shell Tanks B110, U110 } \\
\text { No. Compound Name }\end{array}$ & $\begin{array}{r}\text { Mean } \\
\text { Recovery }\end{array}$ & STD $(N-1)$ & $-3 \times S T D$ & $+3 \times S T D$ \\
\hline 1) $* 1,4-$ Dichlorobenzene-d4 & 100.0 & 0.0 & 100.0 & 100.0 \\
\hline 2) \#2-Fluorophenol & 46.6 & 11.4 & 12.3 & 80.9 \\
\hline 3) \#Phenol-d5 & 54.5 & 11.7 & 19.3 & 89.7 \\
\hline 4) Phenol & 54.2 & 8.7 & 28.2 & 80.2 \\
\hline 5) bis (2-Chloroethyl)ether & 56.3 & 11.3 & & \\
\hline 6) 2-Chlorophenol & 50.5 & 6.6 & 30.8 & 70.2 \\
\hline 7) 1,3-Dichlorobenzene & 53.3 & 13.4 & & \\
\hline 8) 1,4-Dichlorobenzene & 45.6 & 13.3 & 5.6 & 85.6 \\
\hline 9) Benzyl alcohol & 71.0 & 11.4 & & \\
\hline 10) 1,2-Dichlorobenzene & 56.2 & 11.9 & & \\
\hline 11) 2-Methylphenol & 50.7 & 10.3 & & \\
\hline 12) bis (2-Chloroisopropyl)ether & 92.5 & 15.9 & & \\
\hline 13) 4-Methylphenol & 52.3 & 10.1 & & \\
\hline 14) N-Nitroso-di-n-propylamine & 81.8 & 15.2 & 36.1 & 127.4 \\
\hline 15) Hexachloroethane & 30.8 & 14.4 & & \\
\hline 16) *Naphthalene-d8 & 100.0 & 0.0 & 100.0 & 100.0 \\
\hline 17) \#Nitrobenzene-d5 & 59.5 & 12.2 & 22.9 & 96.1 \\
\hline 18) Nitróbenzene & 66.2 & 16.1 & & \\
\hline 19) Isophorone & 55.8 & 25.3 & & \\
\hline 20) 2-Nitrophenol & 62.9 & 8.7 & & \\
\hline 21) 2,4-Dimethylphenol & 27.5 & 21.7 & & \\
\hline 22) Benzoic acid & 37.1 & 16.8 & & \\
\hline 23) bis (2-Chloroethoxy)methane & 76.6 & 11.5 & & \\
\hline 24) 2,4-Dichlorophenol & 64.5 & 8.4 & & \\
\hline 25) 1,2,4-Trichlorobenzerie & 52.8 & 12.3 & 15.9 & 89.6 \\
\hline 26) Naphthalene & 68.6 & 10.0 & & \\
\hline 27) 4-Chloroaniline & 68.1 & 68.7 & & \\
\hline 28) Hexachlorobutadiene & 29.7 & 13.3 & & \\
\hline 29) 4-Chloro-3-methylphenol & 66.7 & 11.2 & 33.1 & 100.3 \\
\hline 30) 2-Methylrıaphthalene & 65.9 & 9.2 & & \\
\hline 31) *Acenaphthene-d10 & 100.0 & 0.0 & 100.0 & 100.0 \\
\hline 32) Hexachlorocyclopentadiene & 14.5 & 8.4 & & \\
\hline 33) 2,4,6-Trichlorophenol & 66.6 & 10.2 & & \\
\hline 34) 2,4,5-Trichlorophenol & 64.9 & 9.9 & & \\
\hline 35) 2-Chloronaphthalene & 56.7 & 10.7 & & \\
\hline 36) $\hbar^{4}$ 2-Fluorobiphenyl & 59.3 & 11.0 & 26.2 & 92.3 \\
\hline 37) 2-Nitroaniline & 84.9 & 15.3 & & \\
\hline 38) Dimethylphthalate & 82.3 & 10.6 & & \\
\hline 39) Acenaphthylene & 62.2 & 12.8 & & \\
\hline 40) 2,6-Dinitrotoluene & 68.1 & 10.6 & & \\
\hline 41) 3-Nitroaniline & 74.0 & 20.3 & & \\
\hline
\end{tabular}


TABLE 33. (contd)

Sample: Single Shell Tanks B110, U110

No. Compound Name

42) Acenaphthene

43) 2,4-Dinitrophenol

44) 4-Nitrophenol

45) Diberizofuran

46) 2,4-Dinitrotoluene

47) Diethylphthalate

48) 4-Chloropheny-phenylether

49) Fluorene

50) 4-Nitroaniline

51) *Phenanthren $\theta-d 10$

52) 4,6-Dinitro-2-methylphenol

53) $\mathrm{N}$-Nitrosodiphenylamine

54) \#2,4,6-Tribromophenol

55) 4-Bromophenyl-phenylether

56) Hexachlorobenzene

57) Pentachlorophinnol

58) Phenanthrene

59) Anthracene

60) Di-n-hutylphthalate

6i) Fluoranthene

62) *Chrysen $\theta-d 12$

63) Pyrene

64) \#Terphenyl-d14

65) Butylbenzylphthalate

66) 3,3'-Dichlorobenzidine

67) Benzo(a)anthracene

68) Chrysene

69) Bis(2-Ethylhexyl) phthalate

70) *Perylene-d12

71) Di-n-octylphthalate

72) Benzo(b) fluoranthene

73) Benzo(k) fluorantitene

74) Benzo(a)pyrene

75) Indeno (1,2,3-cd)pyrene

76) Dibenz $(a, h)$ anthracene

77) Benzo $(g, h, i)$ perylene

Mean

Recovery STD $(\mathrm{N}-1) \quad$-3XSTD +3XSTD

75.

64.3

76.6

68.7

73.5

61.7

129.5

82.8

37.9

100.0

71.9

$1-174$

74.8

67.3

79.5

88.7

81.2

65.3

78.8

100.0

82.4

72.5

83.0

37.0

82.0

83.5

66.9

100.0

67.2

88.7

67.8

63.3

75.5

73.2

74.0

\begin{tabular}{|r|r|r|}
\hline-1$)$ & $-3 \times S T D$ & $+3 X S T D$ \\
\hline 8.7 & 39.7 & 110.6 \\
\hline 15.4 & 30.4 & 122.8 \\
\hline 11.4 & &
\end{tabular}

11.7

$\frac{11.7}{30.2}$

30.2

11.6

\begin{tabular}{|r|r|r|}
\hline 60.0 & & \\
\hline 0.0 & 100.0 & 100.0 \\
\hline
\end{tabular}

NPH (Paraffin Hydrocarbon, C12-C14)

* Compound is an Internal Standard

\# Compound is a Surrogate Spike 


\section{APPENDIX A}

PNL-AL0-120, PROCEDURE FOR EXTRACTION OF SINGLE SHELL TANK SAMPLES FOR THE ANALYSIS OF SEMIVOLATILE ORGANIC COMPOUNDS 
TITLE: PNL-ALO-120, PROCEDURE FOR EXTRACTION OF SINGLE SHELL TANK SAMPLES FOR THE ANALYSIS OF SEMIVOLATILE ORGANIC COMPOUNUS

\section{APPLICABILITY}

This procedure describes the method used for the extraction of single shell tank samples and the subsequent preparation of the extract for the analysis of semivolatile organic compounds. This procedure may be used to support work performed in compliance with EPA Contract Laboratory Program (CLP) or other projects/programs as elected.

\section{DEFINITIONS}

None

\section{RESPONSIBLE STAFF}

Cognizant Scientist

Analyst

\section{PROCEDURE}

1.0 This procedure is for extracting nonvolatile and semivolatile organic compounds from solids such as soils, sludges and other waste samples including single shell tank samples. The sonication process ensures intimate contact of the sample matrix with the extraction solvent. The method is divided into two sections based on the expected concentration of organics in the sample. The low concentration method (individual extractable organic components $\leq 20 \mathrm{mg} / \mathrm{Kg}$ ) uses a larger sample size and a more rigorous extraction procedure. The high concentration method ( $>20$ $\mathrm{mg} / \mathrm{Kg}$ individual extractable organic components) uses a smaller sample, less extraction solvent and is much simpler to perform. The procedure provides for adjustment of $\mathrm{pH}$ for samp?es which do not meet the pH 5-11 extraction criteria. Extracts are filiered and evaporated to a volume suitable for optional GPC cleanup procedures followed by screening and/or analyses by GC/FID or GC/MS.

\subsection{Apparatus}

a) Mortar and pestie.

b) 1-mm standard sieve.

c) Sonicator - Ultrasonic cell disrupter: Heat Systems - U1trasonic, Inc. Model W-385 (475 watt) sonicator or equivalent (power wattage must be a minimum of 375 with pulsing capability) using a no. 208 or no. $3053 / 4$ " disrupter horn for low level analyses, and a no. 200 $1 / 2$ " tapped disrupter horn and no. 419 1/8" standard taper microtip probe.

d) Oven, drying.

e) Desiccator. 
f) Pasteur pipets, 1-ml.

g) Beakers $400-\mathrm{ml}$.

h) Glass scintillation vials, $\rightarrow 20-\mathrm{ml}$, with screw cap and Teflon liners.

i) Buchner funnel

j) Filter paper, Whatman no. 41 or equivalent.

k) Glass conical funnel

1) Filter flask, 500-ml.

m) Concentrator tube, 10-m1 graduated (Kontes K-570050-1025 or equivalent).

n) Evaporator flask, 500-ml (Kontes K-570001-0500 or equivalent).

o) Snyder columns, three-bal1 macro (Kontes K-503000-0121 or equivalent) and two-ball micro.

p) Water bath evaporator, 8-position S-evap, SS boiling water bath Organomation Associates Catalog \#12020 or equivalent.

q) N-evap, 12 position with SS water bath Organomation Assoctates Catalog \#11155 or equivalent.

r) Boiling Chips, 10/40 mesh (silitcon carbide or equivalent) solvent extracted or fired at $450^{\circ} \mathrm{C}$ for 24 hours, cooled in a desiccator and stored in vial with screw cap.

s) Balance, capable of accurately weighing $0.01 \mathrm{gram}$.

t) Spatula, stainless steel or Teflon.

u) Drying column, 20-mm ID. Pyrex ${ }^{R}$ chromatographic column with Pyrex ${ }^{R}$ glass wool at bottom. Note, the glass column may also be fitted with a Teflon stopcock and a fritted glass disc to retain the drying compound, however they are difficult to decontaminate after highly contaminated extracts have been passed through them. Use a smal1 pad of glass wool to retain the drying compound. Prewash the glass wool with $50 \mathrm{ml}$ of acetone followed with $50 \mathrm{ml}$ of elution solvent prior to packing the column with the drying compound.

v) Centrifuge.

w) $\mathrm{pH}$ meter. 


\subsection{Reagents}

a) Sodium sulfate, powdered anhydrous and reagent grade, heated at $450^{\circ} \mathrm{C}$ for 8 hours, cooled in a desiccator and stored in a glass bottle. Baker anhydrous powder, catalog \#73898 or equivalent.

b) Extraction solvents.

c) 1:1 sulfuric acid $\left(\mathrm{H}_{2} \mathrm{SO}_{4}\right)$ and water

d) $10 \mathrm{~N} \mathrm{NaOH}$

\subsection{Standards}

a) Base/neutral acid surrogate solution.

Surrogate standards are added to all samples, blanks, matrix spikes, matrix spike cuplicates, and calibration soiutions; the compounds specified are:

\section{Base Neutrals $(100 \mu \mathrm{g} / 1.0 \mathrm{ml})$ nitrobenzene - $d_{5}$ terphenyl - d 14 2-fluorobiphenyi optional - 1 additional}

\author{
Acids $(200 \mu \mathrm{g} / 1.0 \mathrm{ml})$ \\ plienol $-d_{5}$ \\ 2-fluorophénol \\ 2, 4,6-tribromopheno 1 \\ optiona? - 1 additional
}

b) Matrix standard spiking solutions.

Base/Neutrals $(100 \mu \mathrm{g} / 1.0 \mathrm{ml})$

1,2,4-trichlorobenzene acenaphthene

2,4-dinitrotoluene

pyrene

N-nitroso-di-n-propylamine

1,4-dichlorobenzene
Acids $(200 \mu \mathrm{g} / 1.0 \mathrm{ml})$

pentachlorophenol

phenol

2-chlorophenol

4-ch 1 oro-3-methylphenol

4-nitrophenol

Prepare separate surrogate and matrix spiking solutions at a concentration of $100 \mu \mathrm{g} / 1.0 \mathrm{ml}$ for base/neutral and $200 \mu \mathrm{g} / 1.0 \mathrm{ml}$ for acids in methanol. Store the solutions at $4^{\circ} \mathrm{C}\left( \pm 2^{\circ} \mathrm{C}\right)$ in amber glass Teflon-sealed containers. The solutions must be replaced after twelve months, or sooner, if comparison with quality control check samples indicate a problem.

\subsection{Safety}

a) Observe the general laboratory safety rules.

b) Follow the requirements of applicable RWPs.

c) Observe regulations for handling, storage and disposal of hazardous organic chemicals. 


\subsection{Quality Control}

a) A reagent blank shall be subjected to exactly the same procedure for each set of samples.

b) A matrix spike and matrix spike duplicate [see section 4.4 . (b)] shall be performed on one sample from each set of samples having the same sample matrix and/or to one sample from each group of 20 samples received as a group and/or originating from the same sample matrix. The matrix spike and matrix spike duplicate samples shall be subjected to the same procedure.

c) Base/neutral and acid surrogate spikes [see section 4.4 (a)] shall be added to all blanks, samples, and matrix spiked samples.

\subsection{Analysis}

\subsection{Initial Sample Preparation}

a) Verify that the sample has been received and logged into the Chemistry and Analys is Section per PNL-SA-34.

b) Grind and/or mix the sample choroughly.

c) Weigh out a subsample, (weight shall be specified by the cognizant scientist) for $\mathrm{pH}$ measurement - typically 1 to 2 grams.

d) Add $1 \mathrm{ml}$ of water per each gram of sample and stir for 1 hour.

e) Determine pH of sample with glass electrode and $\mathrm{pH}$ meter while stirring.

Proceed with steps $f$ and $g$ only if the $\mathrm{pH}$ is outside the 5-11 range:

f) Add 1:1 $\mathrm{H}_{2} \mathrm{SO}_{4}$ or $10 \mathrm{~N} \mathrm{NaOH}$ dropwise until the $\mathrm{pH}$ is within the 5-11 range. Allow to stir for 5 minutes. Due to sample variability it is recommended the sample be adjusted to $\mathrm{pH} 7$ 8.

g) Record sample weight, volume of water added to sample, $\mathrm{pH}$, and volume of $1: 1 \mathrm{H}_{2} \mathrm{SO}_{4}$ or $10 \mathrm{~N} \mathrm{NaOH}$ added on the Shielded Analytical Laboratory (SAL) sample preparation instruction sheet (Refer to PNL-SA-50).

h) Dispose of the sample used for the $\mathrm{pH}$ and acid/base adjustment measurement.

\subsection{Extraction of Sample for Medium Level Semivolatile Organics}

a) Weigh out a subsample for extraction. The weight shall be specified by the Cognizant Scientist and shall be recorded on 
the SAL sample prepaiation. (EPA suggested weight for the sample is 1 gram). (Refer to PNL-SA-50)

b) Add sample to a $20-\mathrm{ml}$ vial. Vial shall be cleaned per PNL-SA41 and just prior to the extraction the vial should be rinsed with methylene chloride. If required, add a proportional amount of acid or base as calculated in steps $4.7 .1 \mathrm{f}-\mathrm{h}$. Mix thoroughly into soil

c) Add anhydrous sodium sulfate at twice the weight of the combined sample and $\mathrm{pH}$ adjustment reagents. For 1 gram of sample, add 2 grams of powdered anhydrous zodium sulfate to the sample and mix well.

d) Add $1 \mathrm{ml}$ of the surrogate spike solution to the vial. [See standard section $\$ .4$ (a)]

e) If the sample is chosen for matrix spike or matrix spike duplicate add $1 \mathrm{ml}$ of the matrix standard spiking solution. [See standard section 4.4 (b)]

f) Add whatever volume of methylene chloride needed to bring the final solution volume to $10.0 \mathrm{ml}$ (considering the added volume of surrogate and matrix spike).

g) Disrupt the sample with the $1 / 8$ inch tapered microtip ultrasonic probe for 2 minutes at an output control setting 5 in the continuous mode. Note: before extraction mix the sample and make sure any lumps of the sodium sulfate are broken up and are free flowing using a clean glass rod or a clean spatula.

h) Loosely pack a disposable pipet with 2-3 cm glass wool and 2-3 $\mathrm{cm}$ of granular anhyrous sodium sulfate on top. Do not prerinse the column.

i) Filter the extract through the glass wool and collect $5.0 \mathrm{mi}$ in a concentrator tube. Centrifugation may also be used in place of or in conjunction with step $(j)$.

j) P1ace a micro-snyder column on the concentrator tube. Prewet the Snyder balls by adding a small amount of methylene chloride through the top of the condenser. Gently heat the tube in about a $70-90^{\circ} \mathrm{C}$ water bath. At the proper rate of distillation the balls of the column will chatter continuously; but the chamber will not flood with vapor.

k) When the volume in the concentrator tube is below $-0.5 \mathrm{ml}$ remove the apparatus from the water bath and allow to cool $\sim 10$ minutes. Optionally, the concentrator tube may be placed in an $\mathrm{N}$-evaporator with the water bath at $\left(35^{\circ} \mathrm{C}\right)$ and evaporate the solvent to $0.5 \mathrm{ml}$ using a gentle stream of nitrogen gas. The internal walls of the concentrator tube should be washed down with a small volume of methylene chloride during the operation.

$$
\text { A. } 5
$$


During the evaporation, the tube solvent level must be kept below the water level of the bath. The extract should never be allowed to become dry.

1) Transfer the $0.5 \mathrm{ml}$ concentrate to a $2 \mathrm{ml}$ screw top vial and bring to $1.0 \mathrm{ml}$ from rinsate of the ampule using quantitative transfer. Label the vial with sample ID.

m) Submit the sample for GC/FID screening or GS/MS screening or GS/MS analysis.

n) Fill out the organic extraction bench sheet (PNL-SA-45) and submit it with the sample.

\subsection{Extraction of Sample for Low Level Semivolatile Organics}

a) Weigh out a sample specified by the cognizant scientist. (Note: EPA methodology recommends a $30 \mathrm{gram}$ sample for the low level extraction. Record the sample weight on the SAL sample preparation instruction sheet (Refer to PNL-SA-50).

b) Add the sample to a clean $400 \mathrm{ml}$ beaker. Beaker stiould be cleaned per PNL-SA-41 and just prior to the extraction the beaker should be rinsed with methylene chloride.

c) If the sample pH determined in steps 4.7 .1 a-e is outside the allowable range of $\mathrm{pH} 5-11$, add a proportional amount of $1: 1$ $\mathrm{H}_{2} \mathrm{SO}_{4}$ or $10 \mathrm{~N} \mathrm{NaOH}$ as determined in steps $4.7 .1 \mathrm{f}$-h to obtain ph 7-8. Mix thoroughly.

d) Add 2 times the combined sample and $\mathrm{pH}$ adjustment reagents weight of sodium sulfate. For example, add 60 grams of anhydrous powdered sodium sulfate for a 29 gram sample with 1 gram of acid added.

e) Add $100 \mathrm{ml}$ of $1: 1$ methylere chloride-acetone or a volume equal to 3.3 times the sample weight to the beaker.

f) Add $1.0 \mathrm{ml}$ of the surrogate spike to the beaker [see standards section (a)]

g) If the sample is designated for matrix spiking or matrix spike duplicate, add $1 \mathrm{ml}$ of the matrix standard spiking solution [ see standards section (c)].

h) If the sample extract is to be cleaned up using Gel Permeation Chromatography (GPC) add a volume of spiking solutions specified by the cognizant scientist. Record the volume and source of the spiking solutions on the organic extraction bench sheet (PNL-SA-45).

i) Place the bottom surface of the $3 / 4$ inch sonicator disruptor horn about $1 / 2$ inch below the surface of the solvent. Sonicate for $11 / 2$ minutes with the $W-385$ using no. $2083 / 4$ inch

\section{A. 6}


standard disruptor horn with the output control knob set at 10 (or no. $3053 / 4$ inch tapped high gain Q disruptor horn at 5) and mode switch on "1 sec pulse" and \% duty cycle at $50 \%$.

j) Decant the filter extract through a \#41 filter paper approximately half filled with granular sodium sulfate in a glass conical funnel. Centrifugation may also be used instead of or in conjunction with the above filtration. A sodium sulfate drying column must be used if the funnel drying method is not employed.

k) Repeat the above extraction with 2 more $100 \mathrm{ml}$ or a volume equal to 3.3 times the sample weight portions of the $1: 1$ methylene chloride-acetone mixture.

1) Transfer the extracts to a Kuderna-Danish (K-D) concentrator consisting of a $10 \mathrm{ml}$ concentrator tube, a 3 ball Snyder column and $500 \mathrm{mi}$ evaporative flask. The concentrator should be cleaned per PNL-SA-41 and just prior to assembly should be rinsed with methylene chloride. One or two clean boiling chips should be added to the concentrator tube during the assembly to prevent bumping.

m) P1ace the K-D apparatus on a hot water bath $\left(80\right.$ to $\left.90^{\circ} \mathrm{C}\right)$ so that the concentrator tube is partially immersed in the hot water and the entire lower rounded surface of the flask is bathed with hot steam. Prewet the Snyder balls by adding a small amount of methylene chloride through the top of the condenser. At the proper rate of distillation the balls of the column will chatter continuousiy; but the chamber will not flood with vapor.

n) When the volume in the concentrator tube is below $\sim 5 \mathrm{ml}$ remove the K-D apparatus from the water bath and allow to cool -10 minutes.

0) If screening is required, make the volume of the residue to 10 $\mathrm{ml}, 1 \mathrm{ml}$ of the solution in the concentrator tube can be transferred to an autosampler and/or $2 \mathrm{ml}$ vial and submitted for GC/FID screening or GC/MS screening. Normally low level extracts are concentrated as follows:

p) Place a micro-snyder column on the concentrator tube. Prewet the Snyder balls by adding a small amount of methylene chloride through the top of the condenser. Gently heat the tube in about a $70-90^{\circ} \mathrm{C}$ water bath. At the proper rate of distillation the balls of the column will chatter continuously; but the chamber will not flood with vapor.

q) When the volume in the concentrator tube is below $\sim 0.5 \mathrm{ml}$ remove the apparatus from the water bath and allow to cool -10 minutes. Optionally, the concentrator tube may be placed in an $\mathrm{N}$-evaporator with the water bath at $\left(35^{\circ} \mathrm{C}\right)$ and evaporate the solvent to $0.5 \mathrm{ml}$ using a gentle stream of nitrogen gas. The

\section{A. 7}


internal walls of the concentrator tube should be washed down with a small volume of methylene chloride during the operation. During the evaporation, the tube solvent level must be kept below the water level of the bath. The extract should never be allowed to become dry.

r) Transfer the $0.5 \mathrm{ml}$ concentrate to a $2 \mathrm{ml}$ screw top vial and bring the volume to $1.0 \mathrm{ml}$ using rinsates from the concentrator ampule, use quantitative transfer. Label the vial with sample ID.

Submit the sample for GC/FID screening, GC/MS screening or GC/MS analysis.

s) Fill out the organic extraction bench sheet (PNL-SA-45) and submit it with the sample.

\section{REFERENCES}

USA EPA Contract Laboratory Program, Statement of Work for Organic Analysis, $2 / 88$.

USA EPA Test Methods for Evaluating Solid Waste, SW-846, 3rd Edition. 


\section{APPENDIX B}

PNL-ALO-122, CLEANUP OF HYDROCARBON CONTAINING SAMPLES FOR THE ANALYSIS OF SEMIVOLATILE ORGANIC COMPOUNDS, ReVision 1.0 


\section{PNL TECHNICAL PROCEDURE}

TITLE: PNL-ALO-122, CLEANUP OF HYDROCARBON CONTAINING SAMPLES FOR THE ANALYSIS OF SEMIVOLATILE ORGANIC COMPOUNDS, REVISYON 1.0

\section{APPLICABILITY}

This procedure is useful for the analys is of semivolatile compounds in samples containing interfering quantities of normal hydrocarbons. This procedure was developed to remove normal paraffin hydrocarbons (NPH), typically $C_{12}$ through $C_{14}$, often used as a non-miscible hyárostatic fluid for obtaining radioactive tank waste samples on the Hanford sita. Previously, diluting the sample to reduce the quantity injected into the analytical system has been the only available method of dealing with high concentration levels of hydrocarbons. often the dilution required may be several orders of magnitude which also dilutes the analytes of interest and affects detection limits substantially. Use of this procedure in conjunction with most GC/MS methods does not recluire dilution and allows the stated detection limits to remain unchanged. Any sample matrix containing excessive quantities of normal hydrocarbons, such as waters, sorbents, or soils, are applicable to this procedure.

\section{DEFINITIONS}

NPH (Normal Paraffin Hydrocarbons)

CRQL (Contract Required Quantitation Limits)

\section{RESPONSIBLE SI HFF}

Cognizant Scientist

Analyst

\section{PROCEDURE}

\subsection{ST,OPE AND APPLICATION}

1.1 This method is employed following extraction of a waste or soil using other procedures such as PNL-ALO-120 or -344. In this procedure, residues from PNL-ALO-120 or -344 are partitioned into their base-neutral and acidic components. The base-neutral fraction is cleaned using a silica gel column. Analysis of the cleaned fraction is per other methods such as PNL-ALO-345. Unless specifically mentioned within this procedure, there are no further deviations of the methods used to perform extractions or to analyze residues from this preparation.

1.2 This method may be used for the analysis of radioactive storage tank wastes which contain hydrocarbons in excessive quantity. This procedure does not allow for distinction between hydrocarbons introduced during the sampling process as contamination and those which are native to the tank wastes. An attempt to quantify any 


\section{PNL TECHNICAL PROCEDURE}

compound not included in the Semivolatiles Target Compound List (TCL) following use of this procedure without further validation is inappropriate.

1.3 This procedure may be used to reilove 011 from various matrices, such as soil. Before using this procedure, validation employing matrix spikes and establishing control limits for each distinct matrix should be considered.

\subsection{SUMMARY OF METHOD}

An aliquot of the sample residue produced from a preceding extraction procedure is added to a volume of reagent water. The $\mathrm{pH}$ is adjusted to $>11$ and the base-neutral fraction is extracted into methylene chloride. The $\mathrm{pH}$ of the aqueous phase is aga, $n$ adjusted to $<2$ and the acid fraction is extracted into methylene chloride. Following concentration and exchange into cyclohexane, the base-neutral fraction is eluted through a silica gel (an amorphous silica with weakly acidic properties) column. The hydrocarbon fraction is discarded, followed by collection of the analyte fraction of interest. After concentration, the residue is now ready for GC/MS analysis along with the acid fraction.

\subsection{INTERFERENCES}

3.1 Method interferences may be caused by contaminated solvents, reagents, or glassware. Particular care should be taken when plastic labware of any kind is in the laboratory. Substantial quantities of various phthalate esters, used as plasticisers, can contaminate the sample even from a small amount of solvent exposed to plastic surfaces.

3.2 If pre-packed silica cartridges are substituted for glass columns, it is imperative that the manufacturers lot is checked for contamination. Phthalate contamination is a primary obstacle using prepacked commercial columns.

\subsection{APPARATUS AND MATERIALS}

4.1 Balance: Analytical, capable of accurately weighing 0.01 gram.

4.2 Vials: Pre-cleaned $1.5 \mathrm{~mL}$ amber screw top with teflon lined closures.

4.3 Pipets: Pasteur type or equivalent. 


\section{PNL TECHNICAL PROCEDURE}

\subsection{Kuderna-Danish (KD) Apparatus:}

4.4.1 Concentrator tube: 10 or $25 \mathrm{~mL}$ with ground glass fitting and stopper.

4.4.2 Evaporation flask: KD type, $500 \mathrm{~mL}$.

4.4.3 Snyder columns: Three ball macro and two ball micro or equivalent.

4.7 Oven: Capable of maintaining $150^{\circ} \mathrm{C}$.

4.8 Water bath: Capable of temperature control and boiling water.

4.9 Boiling chips: Cleaned silicon carbide or equivalent.

4.10 Erlenmeyer flasks: $250 \mathrm{~mL}$ and as needed.

4.11 Beakers: $500 \mathrm{~mL}$ and as needed.

4.12 Chromatographic column: $250 \mathrm{~mm}$ long $\times 10 \mathrm{~mm} \mathrm{I.D.} \mathrm{with} \mathrm{pyrex} \mathrm{glass}$ wool plug and Teflon stopcock or equivalent, including commercially available pre-packed cartridges.

4.13 Muffle furnace: Capable of maintaining $400^{\circ} \mathrm{C}$ for several hours.

4.14 Separatory funnel: $125 \mathrm{~mL}$ with teflon stopcock.

4.15 Culture tube: Disposable $16 \times 150 \mathrm{~mm}$ with screw top teflon lined closures.

4.16 Centrifuge: Bench top with openings capable of holding $16 \times 150 \mathrm{~mm}$ culture tubes and achieving $2000 \mathrm{rpm}$.

\subsection{REAGENTS}

5.1 Sodium Sulfate: Granular, anhydrous purified by heating at $400^{\circ} \mathrm{C}$ for at least 4 hours.

5.2 Water: In which an interferant is not observed at or above the CRQL for each parameter of interest.

5.3 Sodium hydroxide solution: $10 \mathrm{~N}$, dissolve 40 grams sodium hydroxide into reagent water and dilute to $100 \mathrm{~mL}$.

5.4 Sulfuric acid solution: A.C.S grade or equivalent sulfuric acid and reagent grade water $1 / 1(\mathrm{~V} / \mathrm{V})$. 


\section{PNL TECHNICAL PROCEDURE}

5.5 Solvents: Cyclohexane, pentane, methylene chloride (pesticide quality or equivalent).

5.6 Sllica gel: $100 / 200$ mesh activated at $140^{\circ} \mathrm{C}$ fo. at least 16 hours in a beaker covered with foil. The stlica gel should not exceed a $5 \mathrm{~cm}$ depth.

\subsection{SAMPLE COLLECTION, PRESERVATION, AND HANDI.ING}

6.1 Samples to be used for this procedure shall follow guidelines provided by the appropriate procedure and matrix, for example PNL. ALO-344.

\subsection{PROCEDURE}

7.1 Acid/Base-neutral partitioning: Proceed to 7.2 when cleaning water extracted residues.

7.1.1 Quantitatively transfer the sample extract obtained from the appropriate extraction procedure such as PNL-ALO-344 or -120 into a clean $16 \times 150 \mathrm{~mm}$ culture tube. Add $10 \mathrm{~mL}$ of reagent water.

7.1.2 Check the $\mathrm{pH}$ of the sample with wide range $\mathrm{pH}$ paper and adjust to $\mathrm{pH}>11$ with sodium hydroxide solution.

7.1.3 Add $5 \mathrm{~mL}$ of methylene chloride to the culture tube. Extract the sample by shaking the culture tube for two minutes with periodic venting to release excess pressure.

7.1.4 Allow the organic layer to separate from the water phase for a minimum of 10 minutes. If the emulsion interface between layers is more than one-third the volume of the solvent layer, then centrifuging should be employed to mechanically aid the phase separation. If centrifuging is unsuccessfuT, the contents of the culture tube may be transferred to a $125 \mathrm{~mL}$ separatory funnel followed by an addition of $50 \mathrm{~mL}$ reagent water. All subsequent extractions are then performed using $20 \mathrm{~mL}$ of methylene chloride.

7.1.5 Collect the lower methylene chloride layer containing the base-neutral fraction using a pasteur pipet and transfer to a concentrator tube. 


\section{PNL TECHNICAL PROCEDURE}

Repeat steps 7.1.3 through 7.1.5 two additional times. Save the base-neutral fraction for solvent exchange, Section 7.2.

7.1.6 Adjust the aqueous phase to $\mathrm{pH}<2$ using the sulfuric acid solution. Perform three methylene chloride extractions as described in steps 7.1 .3 through 7.1 .5 collecting the actd fraction of the partition.

7.1.7 Add a botling chip and concentrate the collected acid fractions using a inicro KD apparatus. For analysis using PNL-ALO-345, when the apparent volume of the residue reaches $0.5 \mathrm{~mL}$, about 5-20 minutes, remove the $\mathrm{KD}$ apparatus and allow tt to drain and cool for at least 10 minutes.

7.1.8 Remove the micro Snyder column and rinse its lower joint into the concentrator tube with a minimum amount of methylenê chloride. Further, centrifuging may be required to remove small particulates in the residue if the radioactivity level exceeds desired levels. Adjust the volume to $1.0 \mathrm{~mL}$. Transfer the residue to a $1.5 \mathrm{~mL}$ amber screw top vial. Cap with a teflon lined closure. Proceed with the appropriate analysis on the alitd residue.

\subsection{Solvent exchange:}

7.2.1 Transfer the sample extract obtained from the base-neutral partitioning procedure to a KD concentrator tube. Add a boiling chip.

7.2.2 Add $4 \mathrm{~mL}$ of cyclohexane and attach a micro Snyder column to the concentrator tube.

7.2 .3 Place the assembled micro $\mathrm{KD}$ apparatus in bolling water so that the concentrator tube is partially immersed in the water.

7.2.4 When the apparent volume of the cyclohexane residue reaches $0.5 \mathrm{~mL}$, about 5-20 minutes, remove the $\mathrm{KD}$ apparatus and allow it to drain and cool for at least 10 minutes.

7.2.5 Remove the micro Snyder column and rinse its lower joint into the concentrator tube with a minimum amount of cyclohexane. Adjust the volume to about $2 \mathrm{~mL}$. 


\section{PNL TECHNICAL PROCEDURE}

\subsection{Column cleanup:}

7.3.1 Prepare a slurry of 10 grams of activated silica gel in methylene chloride and place this into a $10 \mathrm{~mm} \mathrm{I.D.}$ chromatographic column. Tap the column several times to settle the silica gel. Allow $3-4 \mathrm{~cm}$ of unpacked space to remain in the column.

7.3.2 Elute the methylene chloride to $1-2 \mathrm{~cm}$ above the silica ge1. Add $1-2 \mathrm{~cm}$ of anhydrous sodium sulfate to the top of the silica gel.

7.3.3 If using commercial pre-packed columns, begin their use here. Pre-elute the column with $40 \mathrm{~mL}$ of pentane. The rate for all elutions should be about $2 \mathrm{~mL} / \mathrm{minute}$. Discard the pentane eluate.

7.3.4 Just prior to exposure of the sodium sulfate layer to the air, transfer the $2 \mathrm{~mL}$ of cyclohexane base-neutral sample residue onto the column. Use an additional $2 \mathrm{~mL}$ of cyclohexane to rinse and complete the transfer. Continue to discard the column eluent.

7.3.5 Just prior to exposure of the sodium sulfate layer to the air, add $25 \mathrm{~mL}$ of pentane and continue elution of the column. Discard the pentane eluate.

7.3.6 Just prior to exposure of the sodium sulfate layer to the air, add $25 \mathrm{~mL}$ of $2 / 3(\mathrm{~V} / \mathrm{V})$ methylene chloride/pentane. Collect this eluent into a $250 \mathrm{~mL}$. erlenmeyer flask or directly into a $500 \mathrm{~mL}$ KD flask equipped with a concentrator tube.

7.3.7 Just prior to exposure of the sodium sulfate layer to the air, add $25 \mathrm{~mL}$ of methylene chloride to the column. Continue to collect the column eluent.

7.3.8 Just prior to exposure of the sodium sulfate layer to the air, add $25 \mathrm{~mL} 1 / 1(\mathrm{v} / \mathrm{v})$ of methylene chloride/acetone to the column.

7.3.9 Just prior to exposure of the sodium sulfate layer to the air, add $25 \mathrm{~mL}$ of acetone to the column. Collect the eluent unt $i 1$ the column runs dry.

7.3.10 Concentrate the column eluent using the macro KD apparatus, followed by the micro KD apparatus if necessary, to whatever volume is required by the analysis procedure, for 
PNL TECHNICAL PROCEDURE

PNL-AL0-345 discontinue concentration when the apparent volume is $0.5 \mathrm{~mL}$.

7.3.11 Remove the Snyder column and rinse the lower joint and KD flask into the concentrator tube. Adjust the final volume to $1.0 \mathrm{~mL}$ with methylene chloride. Transfer the residue to a $1.5 \mathrm{~mL}$ amber screw top vial. Cap with a teflon lined closure: Proceed with the appropriate analysis on the residue.

\subsection{QUALITY CONTROL}

8.1 Before processing samples, the analyst shall demonstrate through the analysis of a method blank that all glassware and reagents are interference free when used in the quantity as specified in this method. With each batch of samples, and whenever there is a change in reagents or the location of performing this procedure, a method blank shall be processed as a safeguard against chronic laboratory contamination. The blank samples shall be carried out through the all stages of the sample preparation including matching handiing and storage times and location.

8.2 Standard quality assurance practices consistent with the analys is method shall be employed while using this method. Fortified samples shall be carried through all stages of sample preparation adhering to those parameters previously specified with the blank preparation.

8.3 Limits of uncertainty for all measurements shall be in accordance with PNL QA Plan MCS-033 Rev. 0 . Briefly, the limit is \pm 5 in the next digit beyond the last one stated. For example, $5.0 \mathrm{~mL}$ means $5.0 \pm 0.05 \mathrm{~mL}$.

\subsection{Control Limits:}

8.4.1 Surrogate standard determinations shall be performed on all samples and blanks. All samples and blanks are fortified with surrogate spiking compounds before extraction and this cleanup procedure in order to monitor the preparation and analysis of samples.

8.4.2 Matrix spikes shall be performed at a frequency specified by the appropriate method such as PNL-ALO-344 or -120 . Briefly, matrix spike and matrix spike duplicate analyses are required for each matrix and every twenty samples, the same as specified by the E.PA-CLP SOW dated $2 / 88$. 
8.4.3 Control limits for this method were calculated in accordance with EPA and PNL QA PIan MCS-033 Rev 0 procedures. Table 1 provides surrogate recovery control limits and Table 2 provides spiking compound recovery limits obtained from analyses using PNL-ALO-345. Due to the limited quantity of data provided from validation study samples and sample matrices, all control limits for this method shall be considered advisory only. Currently, no action will be required if these control limits can not be met, however, these instances shall be noted in the case narrative. Additionally, these control limits were obtained from calculations which were estimated based on only two representative samples. 
PNL TECHNICAL PROCEDURE

\section{TABLE 1. SURROGATE RECOVERY CONTROL LIMITS}

Surrogate Compound

Nitrobenzene $-d_{5}$

2-Fluorobiphenyl

$p$-Terphenyl-d 14

Phenol- $d_{5}$

2-Fluorophenol

2, 4,6-Tribromophenol

$$
\begin{aligned}
& \text { Leve } 7 * \\
& 100 \mathrm{ug} \\
& 100 \mathrm{ug} \\
& 100 \mathrm{ug} \\
& 100 \mathrm{ug} \\
& 100 \mathrm{ug} \\
& 100 \mathrm{ug}
\end{aligned}
$$

\begin{tabular}{|c|c|c|}
\hline $\begin{array}{l}\text { Matrix Spike Compound } \\
1,2,4-\text { Trichlorobenzene } \\
\text { Acenaphthene } \\
\text { 2,4-Dinitrotoluene } \\
\text { Pyrene } \\
\text { N-Nitroso-Di-n-Propylamine } \\
\text { 1,4-Dichlorobenzene } \\
\text { Pentachlorophenol } \\
\text { Phenol } \\
\text { 2-Chlorophenol } \\
\text { 4-Chloro-3-Methylphenol } \\
\text { 4-Nitrophenol }\end{array}$ & $\begin{array}{l}\text { Leve } 7 \\
100 \mathrm{ug} \\
100 \mathrm{ug} \\
100 \mathrm{ug} \\
100 \mathrm{ug} \\
100 \mathrm{ug} \\
100 \mathrm{ug} \\
100 \mathrm{ug} \\
100 \mathrm{ug} \\
100 \mathrm{ug} \\
100 \mathrm{ug} \\
100 \mathrm{ug}\end{array}$ & $\begin{array}{c}\text { Recovery Limit }(\%) \\
25-81 \\
58-92 \\
56-91 \\
64-101 \\
49-114 \\
15-76 \\
39-138 \\
29-79 \\
31-70 \\
37-96 \\
30-123\end{array}$ \\
\hline
\end{tabular}

\begin{tabular}{cc} 
Recovery Limit $(\%)$ & RPD Limit \\
\cline { 1 - 2 } $34-85$ & 50 \\
$34-84$ & 50 \\
$51-94$ & 50 \\
$20-89$ & 50 \\
$12-81$ & 50 \\
$35-99$ & 50
\end{tabular}

A11 limits are for advisory purposes only

* At time of injection in the validation study. Actual levels used in the procedure are typically 100-200 for acids and 100-50 ug for base-neutrals.

\section{TABLE 2. MATRIX SPIKE RECOVERY LIMITS}

All limits are for advisory purposes only * At time of injection in the validation study. Actual levels used in the procedure are typically 100-200 for acids and 100-50 ug for base-neutrals. 
PNL TECHNICAL PROCEDURE

IABLE 3. METHOD ACCURACY AND PRECISION

\begin{tabular}{|c|c|c|c|}
\hline & Compound Name & Mean & Standard Deviation $(n-1)$ \\
\hline 1$)$ & *1,4-Dichlorobenzene-d4 & 100.0 & 0.0 \\
\hline 2) & \#2-Fluorophenol & 46.6 & 11.4 \\
\hline 3) & \#Phenol-d5 & 54.5 & 11.6 \\
\hline 4) & Phenol & 54.2 & 8.4 \\
\hline 5) & bis(2-Chloroethy 1 )ether & 56.3 & 6.8 \\
\hline 6) & 2-Chlorophenol & 50.5 & 6.6 \\
\hline 7) & 1,3-Dichlorobenzene & 53.3 & 10.9 \\
\hline 8) & 1,4-Dichlorobenzene & 45.6 & 10.2 \\
\hline 9) & Benzyl alcohol & 71.0 & 7.4 \\
\hline 10) & 1,2-Dichlorobenzene & 56.2 & 8.4 \\
\hline 11) & 2-Methylphenol & 50.7 & 7.4 \\
\hline 12) & bis (2-Chloroisopropyl) ether & 92.5 & 10.8 \\
\hline 13$)$ & 4-Methylphenol & 52.3 & 8.4 \\
\hline 14) & N-Nitroso-di-n-propylamine & 81.8 & 10.8 \\
\hline 15) & Hexachloroethane & 30.8 & 11.6 \\
\hline 16) & *Naphthalene-d8 & 100.0 & 0.0 \\
\hline 17) & \#Nitrobenzene-d5 & 59.5 & 8.6 \\
\hline 18) & Nitrobenzene & 66.2 & 5.4 \\
\hline 19) & Isophorone & 55.8 & 23.7 \\
\hline 20) & 2-Nitrophenol & 62.9 & 8.7 \\
\hline 21) & 2,4-Dimethylphenol & 27.5 & 5.7 \\
\hline 22) & Benzoic acid & 37.1 & 16.8 \\
\hline 23) & bis(2-Chloroethoxy)methane & 76.6 & 5.9 \\
\hline 24) & 2,4-Dichlorophenol & 64.5 & 8.4 \\
\hline 25) & 1,2,4-Trichlorobenzene & 52.9 & 9.2 \\
\hline 26) & Naphthalene & 68.6 & 5.9 \\
\hline 27) & 4-Chloroaniline & 68.2 & 14.4 \\
\hline 28) & Hexachlorobutadiene & 29.7 & 10.1 \\
\hline 29) & 4-Chloro-3-inethylphenol & 66.7 & 9.9 \\
\hline 30$)$ & 2-Methylnaphthalene & 65.9 & 4.6 \\
\hline 31) & *Acenaphthene-d10 & 100.0 & 0.0 \\
\hline 32) & Hexachlorocyclopentadiene & 14.5 & 8.4 \\
\hline 33) & 2,4,6-Trichlorophenol & 66.6 & 10.2 \\
\hline 34) & 2,4,5-Trichlorophenol & 64.9 & 9.9 \\
\hline 35) & 2-Chloronaphthalene & 56.7 & 5.3 \\
\hline 36) & \#2-Fluorobipheny? & 59.3 & 8.4 \\
\hline 37 & 2-Nitroaniline & 84.9 & 7.9 \\
\hline 38) & Dimethylphthalate & 82.3 & 6.6 \\
\hline 3 & Acenaphthylene & 62.2 & 5.5 \\
\hline 4( & 2,6-Dinitrotoluene & 68.1 & 5.3 \\
\hline & 3-Nitroaniline & 73.9 & 6.2 \\
\hline
\end{tabular}


PNL TECHNICAL PROCEDURE

TABLE 3. METHOD ACCURACY AND PRECISION (continued)

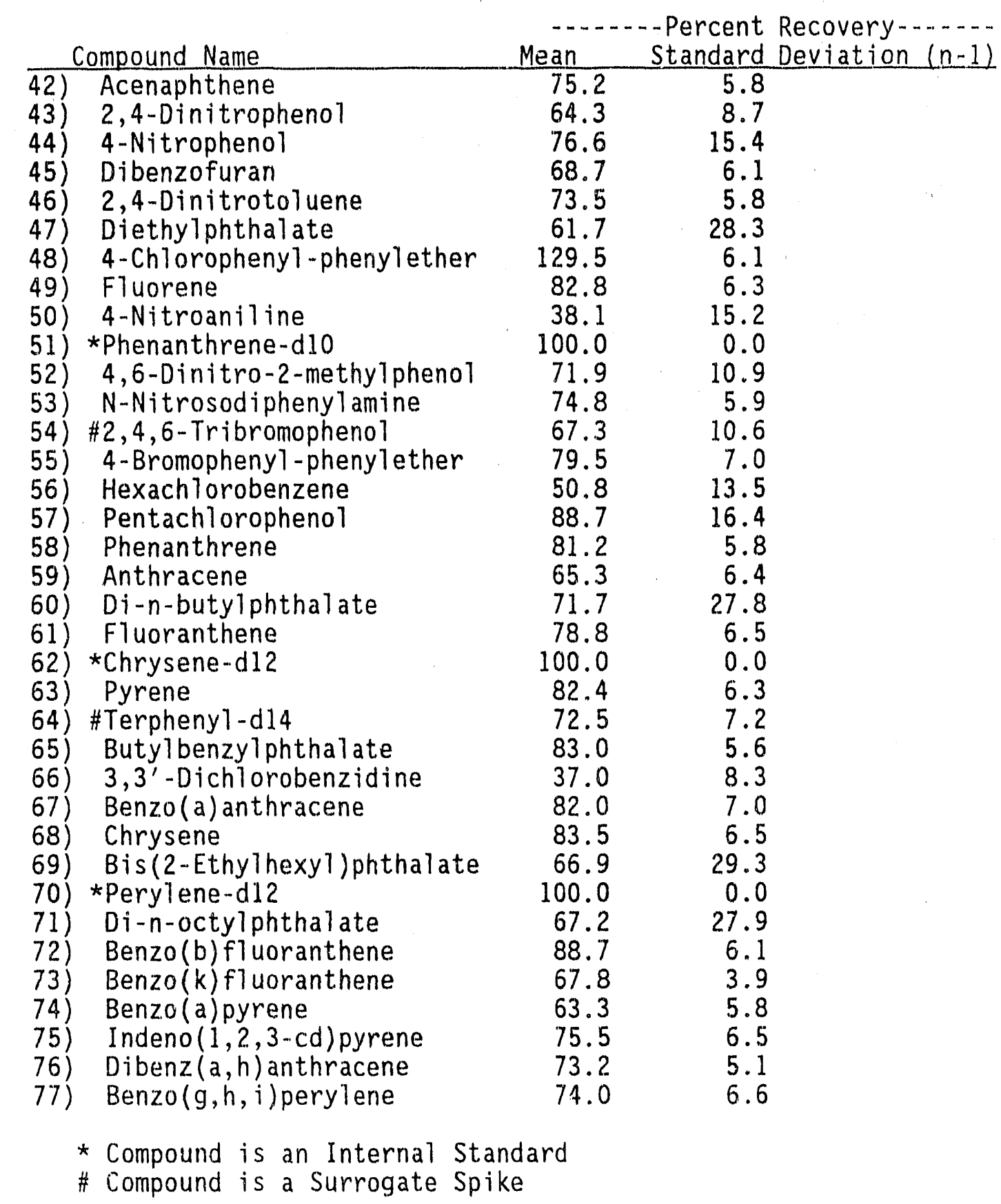


PNL TECHNICAL PROCEDURE

\subsection{METHOD PERFORMANCE AND SPECIFIC QUALIFICATION}

9.1 Table 3 provides method accuracy and precision based on analysts of:

22 acid surrogate spiked samples

12 base-neutral surrogate spiked samples

17 acid TCL spiked samples

9 base-neutral TCL spiked samples

These data were obtained from three different matrices. As seen from $\{a b l e s 1,2$, and 3 , the base-neutral ranges are quite wide. The low number of determinations contributes substantially to variability. It is anticipated that the range will narrow when more data is available.

9.2 The validation of this procedure was performed using spikes of the complete Semivolatiles Target Compound List (TCL) from the USE.PACLP, SOW dated $2 / 88$, on a limited number of authentic single shell tank wastes and blank samples. See reference 1.

\section{REFERENCES}

1. Hoppe, E. W.; Stromatt, R. W.; Campbe11, J. A., Steele, M. J., Jones, T. E., Development and Validation of a Cleanup Method for Hydrocarbon Containing Samples for the Analysis of Semivolatile Organic Compounds, Pending Battelle-PNL Technical Report, 4/92.

2. PNL-ALO-120, Semivolatile Organic Extractions of Single Shell Tank Samples, Rev. 0, Battelle-PNL Technical Procedure, 8-3-89.

3. PNL-AL0-344, Procedure for Sample Preparation of Samples for GC/MS Analys is for Semivolatile Organic Compounds, Rev. 0, Battelle-PNL Technical Procedure, 4-26-91.

4. PNL-ALO-345, GC/MS Analys is of Extractable Semivolatile Organic Compounds, Rev. 0, Battelle-PNL Technical Procedure, 8-3-89.

5. QA Plan for Activities Conducted by the Analytical Chemistry Laboratory (ACL), QA PIan MCS-033, Rev. 0, 9-4-91

6. USEPA Contract Laboratory Program, Statement of Work for Organic Analysis, Multi-Media, Multi-Concentration, SOW No. 2-88. 
7. USEPA Contract Laboratory Program, Statement of Work for Organic Analysis, Multi-Media, Multi-Concentration, Document Number OLM01.0, March 1990.

8. USEPA Office of Solid Waste, Iest Methods for Evaluating Solid Waste, SW-846, Third Edition, 11-86. 


\section{DISTRIBUTION}

No. of

Copies

\section{OFFSITE}

12 DOE Office of Scientific and Technical Information

\section{ONSITE}

\section{DOE Richland Field Office}

J. M. Clark, A4-02

J. R. Noble-Dia1, A4-02

11 Westinghouse Hanford Company

D. A. Dodd, T6-50

S. G. Mckinney, T6-50

S. G. Metcalf, T6-50

A. F. Noonan, R2-12

L. M. Sasaki (5), R2-12

K. L. Silvers, T6-08

W. I. Winters, T6-50
No, of

Coptes

15 Pacific Northwest Laboratory

J. A. Campbe 17, P8-08

E. W. Hoppe (5), P7-22

$T$. E. Jones, $P 7-27$

M. J. Steele, P7-53

R. W. Stromatt, P7..22

Publishing Coordination Technical Report Files (5) 

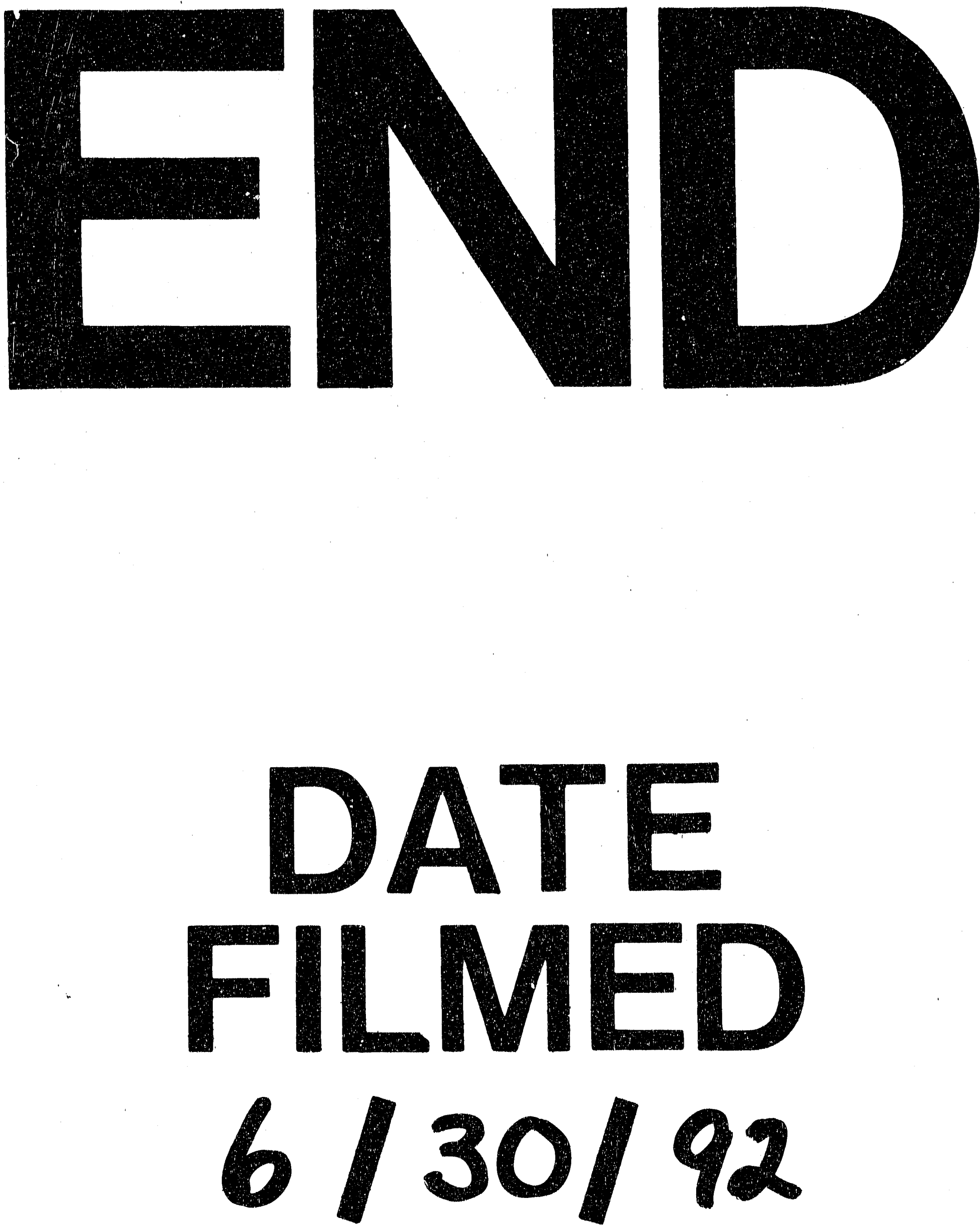
